


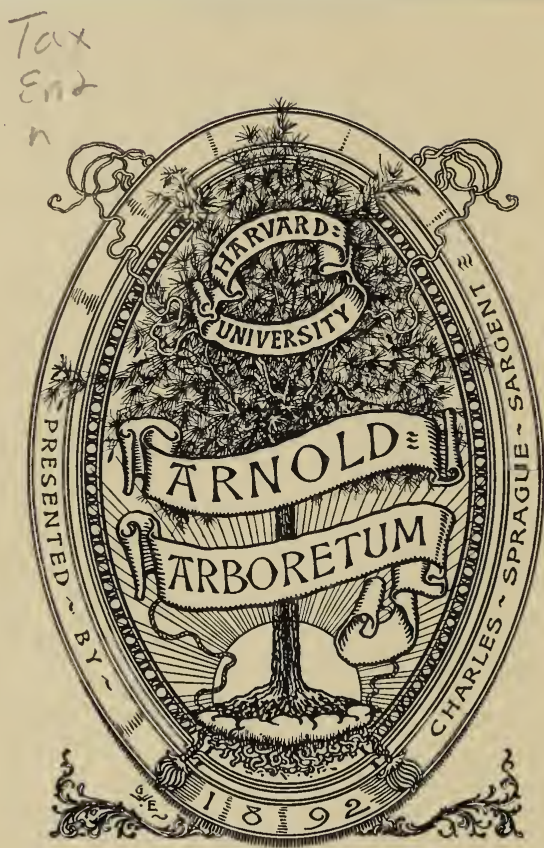



Digitized by the Internet Archive in 2017 with funding from BHL-SIL-FEDLINK 
21,0

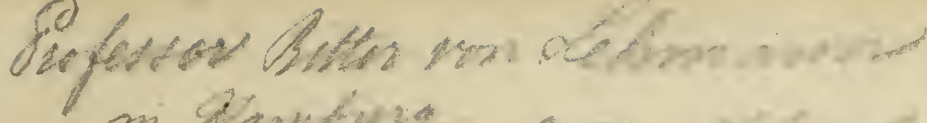
m garnowy

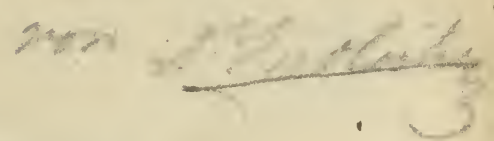

\section{NOVARUM STIRPIUM}

\section{DECAS I-X.}

\section{9.}

$$
\text { Uisidelonar. }
$$




\section{CONSPECTUS DECADIS I-X.}

\section{Haemodoraceae.}

1258. Conostylis juncea Endl. n. 22. - Nov. Holl. Hügel. - Conostylis candicans Endl. n. 23. - Nov. Holl. Hügel.

* 1259/1. Tribonanthes australis Endl.n.34. - Nov. Holı. Hügel.

\section{Grelnidleae.}

1586. Thelymitra flexuosa Endl. n. 29. - Nov. Holl. Hügel. 159'. Glossodia Brunonis Endl. n. 20. - Nov. Holl. Hügel.

\section{Tajad eae.}

* 166t/1. Cycnogeton Hügelii Endl. n. 89. - Nov. Holl. Hügel.

\section{Chenopodeae.}

* 1920/1. Didymanthus Roëi Endl. n. 10. - Nov. Holl. Roë.

1928. Kochia salsoloides Fenzl. n. 85. - C. B. S. Drège.

Santalaceae.

* 2031/1. Glischrocaryon Roëi Endl. n. 88. - Nov. Holl. Roë.

\section{Protenceae.}

2132. Conospermum Hügelii R.Br.n.66. - Nov. Holl. Hügel.

- Conospermum laniflorum Endl.
n. 67.
- Nov. Holl. Hügel.

- Conospermum Stoechadis Endi. n. 68. - Nov. Holl. Hügel.

2142. Anadenia integrifolia Endl. n. 98. - Nov. Holl. Roë.

- Anadenia filiformis Endl. n. 99. - Nov. Holl. Roë.

* 2142/1. Manglesia tridentifera Endl.n.31. - Nov. Holl. Roë.

- Manglesia vestita Endl. n. 32. - - Nov. Holl. Hügel.

2143. Grevillea Thelemanniana Hüg.

n. 8. . . . - Nov. Holl. Hort. Hüg.

\section{Rulbiaceae.}

* 3279/1. Crossopteryx Kotschyana Fenzl.
n. 51
- Afr. trop. Kotschy.

\section{Hognniacene.}

* 3363/1. Coelostylis loganioides Torr. et Gr. n. 41 .

- FInrida. Herb. Torr.

\section{Asclepiadeac.}

* 3t47/1. Conomitra linearis Fenzl. n. 74. - Afr. trop. Kotschy.

* 350t/1. Fockea capensis Endl. n. 21. - - C., B. S. Hort. Schönbr.

\section{Gentianene.}

3551. Irlbachia Bonplandiana Fenzl.

n. 14. •. . . . Nov. Granad. Bonpl.

\section{Verbenaceae.}

369\%/1. Mallophora globiflora Endl.n. 72. - Nov. Holl. Roë. _- Níallophora corymbosa Endl.n.73. - Nov. Holl. Roë.

IIyoporineae.

* 3733/1. Dasymalla axillaris Endl. n. 12. - Nov. Holl. Ro _ Dasymalla terminalis Endl.n.13. - Nov. Holl. Roë. 3734. Pholidia resinosa Endl. n. 58. - - Nov. Holl. Roë. 3735. Eremophila viscida Endl. n. 59. - Nov. Holl. Rö̈. 3736. Stenochilus racemosus Endl.n.57. - Nov. Holl, Rö̈. 


\section{Asperifoliae.}

3742/1. Halgania anagalloides Endl.n.62. - Nov. Holl. Roë.

__ Halgania integerrima Endl.n.63. - Nov. Holi. Roë.

_- Halgania lavandulacea Endl.n.6'. - Nov. Holl. Roë.

* $\overline{3779}$. Friedrichsthalia physaloides Fenzl. n. 61 .

\section{Seroplatalarimae.}

3902. Anthocercis anisantha Endl.n.80. - Nov. Holl. Rö̈. * 3902/1. Anthotroche pannosa Endl. n. 9, - Nov. Holl. Roë.

* 3944. Anticharis arabica Endl.*n. 28. - Arabia felix.Fisch, et Schimp.

\section{Acanthaceac.}

* 4072/1. Russeggera ${ }^{3}$ collina Endl. n. 46. - Africa tropica. Russeg. 4072/2. Schwabea modesta Endl. n,91. - Africa tropica. Kolschy.

\section{Epacrideae.}

4273. Leucopogon corifolius Endl.n. 19. - Nov. Holl. Hort. Hügel.

\section{Unbellifenae.}

* 4357. Cesatia ornata Endl. n. \%1. - - Nov, Holl. Rö̈.

\section{Saxifragaceac.}

* 4650. Aphanopetalum resinosum Endl. n. 42

\section{Portulacene.}

* Plinthus cryptocarpus Fenzl.n.60.- C. B. S, Drège.

* Diplochonium sesuvioides Fenzl. n. 65 .

* Ancistrostigma cypseleoides Fenzl.

n. 94. . . . . . - Nov. Holl. Ferd, Bauer.

* Monocosmia corrigioloides Fenzl. n. 93.

\section{Silleneae.}

Silene 'thysanodes Fenzl. n. 39, - Amer. trop. Fr, Hall.

\section{Plnytolaceeae.}

Semonvillaea fenestrata Fenzl.
n. 48.
C. B. S. Drège.

Limeum telephioides $\dot{F}$ enzl. $n .96$.

Limeum viscosum Fenzl. n. 98. C. B. S. Ecklon.

Giseckia Miltus Fenzl. n. 45 . Senegal. Sieber.

\section{Hasiopetaleac.}

* Corethrostylis bracteata Endl.n. 1. - Nov. Holl. Roë. Thomasia macrocarpa Hüg.n. 40, - Nov, Holl, Hügel.

\section{Dombeyaceac.}

Xeropetalum multiflorum Endl.

n. 43. - . . - Africa tropica Kotschy.

Xeropetalum minus Endl. n. 4', - Africa tropica Kotschy.

Xeropetalum tiliaceum Endl.n.45. - C. B. S. Drège.

\section{Marcgraviacene.}

* Brassaia actinophora Endl.n.100. - Nov. Holl. F. Bauer.

\section{Sapincliaceac.}

Dodonaea humilis Endl. n. 33. . - Nov. Holl. Ferd. Bauer.

* Ploesslea floribunda Endl. n.4\%. - Africa tropica. Kotschy.

\section{Hippocartanae.}

* Ungnadia speciosa Endl. n. 86. - Texas, Drummond. 


\section{Thamneac.}

Pomaderris hirta Reiss, n. 37. - - Nov. Holl. Hügel. Soulangia oppositifolia Reiss.n. 90. - St. Ielena Whelley. Cryptandra pomaderroides Reiss. n. 36. - Nov. IIoll, Rö̈.

* Petalopogon cuspidatus Reiss.n.92. - C. B. S. Drège et Eckl. Crumenaria erecta Riss. $n$. 35. - Brasilia Pohl. Zizyphus Gardneri Reiss, n. 38. - Brasilia Gardner.

\section{Pittosporeac.}

* Pittosporum Hügelianum Putt.

n. 49. . . - Nov. Holl. Hügel.

* Pittosporum densiflorum Putt.n.50.- Ind. or. Hügel.

Billardiera latifolia Putt. n. 52. - Nov. Holl. Ferd Bauer.

Billardiera grandiflora Putt. n.53. - Nov, Holl. Ferd. Bauer.

* Oncosporum bicolor Putt. n. 11. - Nov. Holl. Hort. IIügel. Marianthus erubescens Putt.n. 69. - Nov. Holl Roë. Marianthus floribundus Putt.n.70. - Nov. Holl. Roë.

\section{Eertaceace.}

Boronia ternata Endl. n. 97. . - Nov. Holl. Rö̈.

\section{IN y}

Polyzone purpurea Endl. n. 81. - Nov. Holl. Roë. Thryptomene australis Endl.n. 82. - Nov. Holl. Roë. Chamaelaucium virgatum Endl. - Nov. Holl. Roë. n. 84. . . . . . Nov. Holl. Rö̈.

Verticordia compta Endl. n. 75. - Nov. Holl. Roë. Verticordia Roëi Enăl. n. 76. - Nov. Holl. Roë. Verticordia picta Endl. n. 77. - - Nov, Holl. Roë. Verticordia chrysantha Endl.n.;8. - Nov. Holl. Rö̈. Verticordia grandifiora Endl.n.79. - Nov. Holl. Rö̈. Pileanthus peduncularis Endl.n.83. - Nov. Holl. Rö̈.

\section{Papilionaceac.}

Spadostyles Hügelii Endl. $n$. 3. - Nov. Holl. Hort.Hüg. Spadostyles Benthamii Endl.n. 4. - Nov. Holl. Hort. Hüg. Spadostyles concolor Endl. n.24. - Nov. Holl. Hort. Hüg. Spadostyles ramulosa Endl.n.25. - Nov. Holl. Hort. Hlüg. Pultenaea rosmarinifolia Endl.n.5. - Nov. Holl. Hort. Hüg. Dillwynia elegans Endl. n. 15. - Nov. Holl. Hort. Hüg. Dillwynia filifolia Endl. n. 16. - Nov. Holl. Hort. Hüg. Dillwynia seriphioides Endl.n. 17. - Nov. Holl. Hort. Mäg. Dillwynia adenophora Endl. n.30. - Nov. Holl. Hort. Hüg. Oxylobium spectabile Endl. n. 2. - Nov. Holl. Rö̈. Jacksonia floribunda Endl. n. 87. - Nov. Holl. Rö̈. Daviesia macrophylla Endl. n. 18. - Nov. Holl. Hort. Hüg. Daviesia alternifolia Endl. n. 54. - Nov. Holl. Hügel. Daviesia ternata Endl. n. 55. . - Nov. Holl. Hügel. Daviesia oppositifolia Endl.n.56. - Nov. Holl. Hügel. Bossiaea ovalifolia Endl. n. 26. - Nov. Holl. Hort. Hüg. Bossiaea nummularia Endl.n. 27. - Nov. Holl. Hort. Hüg.

* Kotschya africana Endl. n. 6. - - Africa tropica. Kotschy. 
Nr. 1.

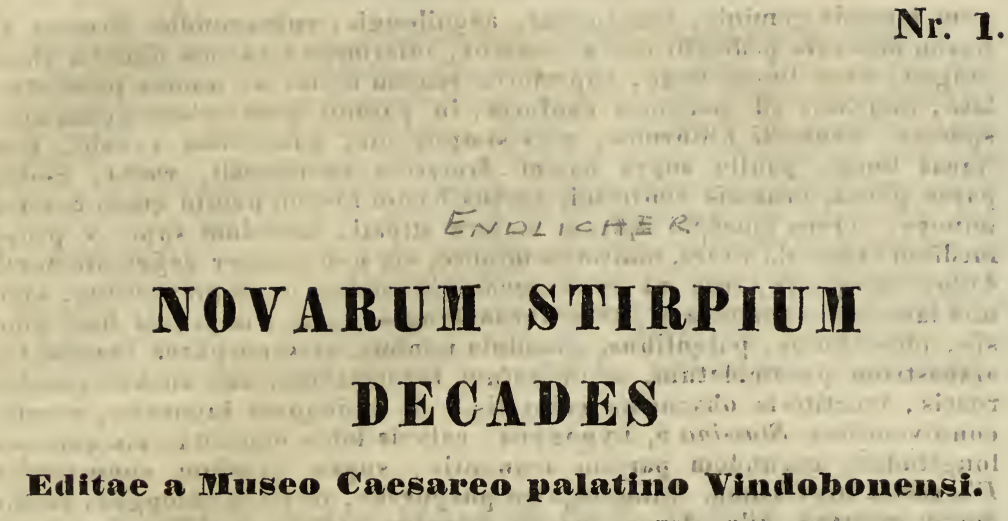

Nr. 1. Vindobonae, typis Sollingerianis. 1. Maji 1839.

CORETHROSTYLIS Endl. Inflorescentia oppositifolia, cymoso-racemosa. Bracteolae a calyce remotae, coloratae. Calyx corollinus, quinquepartitus, persistens. Corolla nulla. Stamina 5, hypogyna, calycis laciniis allerna; filamenta brevissima, libera, antherae ovoideo-oblongae, medio dorso insertae, supra ovarium conniventes, biloculares, loculis intus prope apicem poro apertis. Ovarium triloculare; ovulis in loculis geminis, collateralibus, ex anyuli centralis basi adscendentibus, anatropis. Stylus terminalis, longus, pilorum fasciculis retrorsis hispidus, scopaeformis. Capsula trilocularis, loculicide trivalvis, valvis medio septiferis. Semina in loculis solitaria, e basi erecta, ovato-oblonga, strophiola umbilicali cupulaeformi, multipartita, laciniis filiformibus. Embryo in axi albuminis carnosi orthotropus; cotyledonibus ovatis, radicula tereti, umbilico proxima, infera. - Frutices NovaeHollandiae austro-occidentalis; foliis alternis, cordatis, petialatis, exstipulatis, pilis stellatis consicis, inflorescentia oppositifolia cymoso-racemosa, bracteis foliaceis," bracteolis coloratis. Genus Lasiopetalo proximum.

\section{Corethrostylis bractenta Endl.}

Crescit in Novae-Hollandiae austro-occidentalis interioribus. (Roë.) Colitur in horto Hügeliano.

Frutex, in specimine culto florente bipedalis, ramis teretibus, pube densa, immixtis pilis stellatis, rufescentibus consitus." Folia alterna, tres quartas pollicis partes dissita, petiolata, petiolo tereti, erecto, internodium paullo superante, pilis rufis dense consito, lamina cordata, acutiuscula, coriacea, sesquipollicen longa, latitudine maxina pollicem cum quadrante metiente, margine obsolete repando-subsinuata, nervis subtus prominulis, intra marginem confluentibus, supra obsoletis, pagina superiore pilis stellatis brevibus adspersa et scabriuscula, inferiore pube molli induta, pilisque stellatis, fuscis, in nervis confertioribus conspersa. 'Inflorescentia exacte oppositifolia, pedunculo petiolum paullo superante, in cymam simplicem, folio quidquam longiorem desinente, nempe flore alari 
cum racemis geminis, simplicibus, aequilongis, subsecundis. Bractea ad basim cujusvis pedicelli ovata, sessilis, inferiores tres cum dimidia linea longae, duas lineas latae, superiores sensim minores, omnes pube stellata, imprimis ad margines conferta, in paginis plus minus densa conspersae. Pedicelli filiformes, pilis simplicibus, patentibus consiti, tres lineas longi, paullo supra basim bracteola sublaterali, rosea, molli, parce pilosa, bracteis conformi, versus basim racemi paullo quam bractea minore, versus ejusdem apicen majore stipati, interdum supra v. prope medinm bracteola altera, multoties minore, vix non semper deficiente aucti. Calyx corollinus, fere ut in Keraudreniis mollis, quinquepartitus, laciniis lanceolato-acuminatis, tres lineas longis, extus, imprimis basi pilosis, pilis albidis, patentibus, glandula minima, atro-purpurea terminatis, alabastrum pyramidatum, acuminatum formantibus, sub anthesi patulis, roseis, fructiferis obscurius coloratis, vix quidquam induratis, erectoconniventibus. Stamina 5, hypogyna, calycis lobis opposita, vix quartam longitndinis eorundem partem aequantia, supra ovarium conniventia. Filamentá brevisšnna. Antherae atro-purpureae, ovoideo-oblongae, medio dorso insertae, biloculares, loculis margine juxta totam fere longitudinem quidquam introflexis, intus infra ipsum apicem foramine parvo dehiscentibus. Ovarivion"sessile, globosum, pilis brevibus glanduloso-rapitatis consitum, intus triloculare. Ovula in.loculis gemina, ex anguli centralis basi collateraliter adscendentia, anatropa, umbilico cupula carnosa

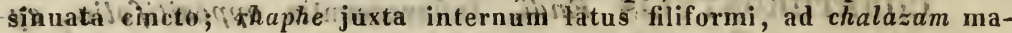
nriltaefotmęn, terminalen, umbilico diametro oppositam pronducta. Stylus, terminalis, ovario plus quadruplo, Longior, calycis dacinias longitudine acquans, filiformi-snbulatus, ima basi nudus, caeterum juxta totam Iongitudinem pilorum candidorum fasciculis dependentim stipitatis indutus, fasciculis versus apicém sensim decrescentibus elongato-conicus, scópaformis. Stigma in "ipso styli apice punctiforme, nudum. Capsula globosa; Pisi dinoris imole, calyce persistente, vix quidquam aucto (et qonnihil indurato, oracta-connivente, basi obsolete pentagono, obscurius colorato induta, antheris persistentilus ima hasi stipata, stylo pilis vit in flore hispido superata, trilocularis, loculicide trivalvis, valvis membranaceis, wlotete pilbsis, sordide stranineis, venosis, medio septiferisi, apice diu stylo conjunctis. Scmind in Toculis solitaria, e basi loculi erecta, ovato-ololongag ntrinque nutusa, dorso convexa, facie subeomplanata testa rubro-fusca, durissima, rhaphe obsoleta, chalaza minutissime mamillaeformi. Strophrola umbilicalis subcarnosa, brevissime cupuliformis, in lacinias $6-8$ filiformes, dimiditum seminis aequantes, saepe inatutulongas fissa. Embryo in axi afluminis dense carnosi orthotropus, ejtisdemifere Iongitudine. Cotyledones watae, planäe. Radicula cylindrica, cotyledonibus paullo longior, umpilicum attingens, infera.

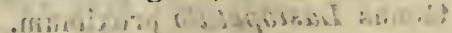

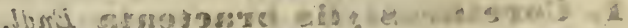

2. Orylobinnm spectabile Endl. O. foliis oppositis late cordatis amplexicauli - subsessilibus glaberiug marginatis mucro natis, racemo terminali.

sors Novae-Hollandiae austro-occidenlalis interiora. (Кö̈.)

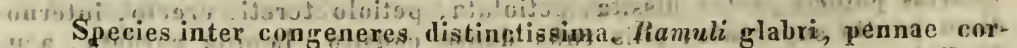
viaae crassitie, internorliis sesquipollicaribus, inferioribus:s teretibus, superioribus alternatim contrarie, compressis, omnibus ad nodos incrasesatis, laevibus, striatis. Folia opposita, subsessilia, petiolo crasso, vix lineam longo, coriacea, cordato-suprotanda, acuta, $1^{1 / 4}$ pollicem longa, $11 / 2$ pollicem lata, Lóbis baseos rotunda tu obtuşissimis, subanplexicautibus, utrinque glaberrima, glauco-viridia, reticulato-venosa, nervo unico, subtus valide prominpate, sursum sensiphathenuato, cui margine an- 
gusto, cartilagineo in mucronem rigidum, pungentem, dimidia linea paullo longiorem coëunte. Stipulae lineari-acuminatae, sesquilineares, nigricantes. Racemus terminalis, sesquipollicaris, pedicellis oppositis ternatisque, sesquilineam longis, glabris, bracteis sub anthesi delapsis. Flores paullo quam in plerisque congenermm majores. Calyx tubo campanulato, sesquilineari, limbi tubum aequantis bilabiati labio superiore bifido, inferiore tripartito; labii superioris lobis obtusiusculis, inferioris acutis, omnibus extus parce puberulis, intus glabris, subcoloratis. Corollae vexillum rotundatum, unguiculatum, $4 \frac{1}{2}$ lin. Iongum, aurantiacum, alae et carina obtusa, lutea. Stamina libera, filamentis filiformibus, glabris. Ovarium brevissime stipitatum, dense-albo villosum, oblongum, ovulis 8-10, confertis. Stylus filiformis, incurvus, glaber; stigma subcapitatum.

3. Spadostyles Firigelii Endl. Sp. foliis alternis oblongolinearibus planis acutis, pedicellis brevibus, bracteolis calyce brevioribus.

\section{Colitur in horto Hügeliano.}

Tota planta pube rara, defluente conspersa. Folia alterna, 6 lineas longa, apice acuta, lineam lata, basi angustata, in petiolum 'dimidiam lineam longum attenuata, mucrone brevi deciduo terminata, floralia conformia. Stipulae petiolum utrinque stipantes paleaceo-setaceae, fuscae, lineam longae, liberae. Flores axillares, solitarii, racemum brevem, apice comosum constituentes, pedicellis sesquilineam-longis, apice incrassatis. Bracteolae stipulis conformes, dimidiam lincam longae, calyci bilineari adpressae. Flores Spadostylis Sieberi Benth., sed quidquam minores. Sty$l u s$ basi manifeste, sed minus quam in aliis speciebus compressus, ibique parce villosus, Ovarium glaberrimum.

4. Spadostyles Benthamii Endl. Sp. foliis alternis oblongo-linearibus dorso convexis facie subconcavis acutis mucronatis, pedicellis brevissimis, bracteolis calycem aequantibus.

\section{Colitur in horto Hügeliano.}

Tota planta glaberrima. Folia 6 lineas longa, vix lineam latitudine aequantia, basi angustata et in petiolum brevissimum attenuata, acuta, mucrone brevi, recto, subcarnosula, dorso convexa, facie subconcava, floralia conformia. Stipulae intraaxillares paleaceae, lanceolatae, acuminatae, liberae, v. foliorum superiorum, imprimis floralium latiores, basi v. juxta totam fere longitudinem in unicam, acuminato - bidentatam connatae. Flores in superiorum foliorum axillis solitarii, pedicellis vix tertiam unius lineae partem superantibus insidentes, capituli comosi speciem referentes. Calyx laxe campanulatus, duas et dimidiam lineam longus. Bracteolae lineares, canaliculatae, subcarnosae, acutae, calycis basi adnatae, eidem aequilongae. Flos paullo major quam in praecedente specie. Ovarium glaberrimum. Stylus valde compressus, ovario vix quidquam angustior, glaberrimus. 
5. Pultenaea rosmanimifolia Endl. P. foliis oblongis mucronatis margine revolutis, subtus adpresse sericeis, capitulis terminalibus densis multifloris, bracteis imbricatis scariosis villosis carinatis, bracteolis calycem aequantibus.

\section{Colitur in horto Hügeliano.}

P. myrtoidi Cunningh. affinis, facile distinguenda: foliis pollicem longis, duas lineas latis, mucrone brevi, subrecurvo superatis, in petiolum $1 / 2$ lineam longum attenuatis, margine revolutis, nervo supra impresso, subtus valde prominente, pagina inferiore adpresse sericea. Stipula intraaxillaris lanceolata, duas lineas longa, acuminata, medio paleacea, carinato-concava, glabra, marginibus villosa. Bracteae involucrantes stipulis conformes, paullo latiores, longius et densius villosae. Calycis laciniae acutissimae, superiores paullo latiores, quidquam breviores, omnes pube sericea subplumosae. Bracteolae setaceae, sericeo-plumosae, dimidio calyci adnatae et aequilongae. Corollac vexillum cum alis aureum, carina atro-purpurea.

KOTSCHYA Endl. Calyx basi Uibracteolatus, bilabiato-bipartitus, labio superiore compresso, emarginato, incumbente, inferiore trifido, patentiusculo. Corolla papilionacea, vexillo oblonyo, alis carinam obtusam, dipetalam aequantibus. Stamina 10, monadelpha. Legumen stipitatum, inclusum, articulatum, articulis secedentibus, monospermis, suborbicularibus, marginatis. Frutex, ramulis alternis, simplicibus, setoso-hispidis, foliis abrupte pinnatis, multijugis, foliolis ovato-oblongis, basi inaequilateris, stipulis scarioso-membranaceis, racemis axillaribus paucifloris, folio brevioribus, floribus nutantibus.

Genus Hedysareum, calycis forma insigne, nominavi in honorem cl. Theodori Kotschy, qui post exploratas Syriae et Asiae minoris gazas botanicas, nuper Russeggero duce Africae orientalis regiones tropicas penetravit, plurimasque, praeter hauc ipsam, stirpes novas detexit.

\section{Kotschya africuna Endl.}

In Africae boreali-orientalis insula fluvii Tumad, in confinibus Schangul et Camamil legit cl. Theodorus Kotschy (n. 524.)

Fruticis ramos video quadri-octopedales, strictos, inferne fere digitum minimum crassos, sursum sensim angustatos, cortice fascescenti stramineo, longitudinaliter rugoso-rimoso vestitos, setis patentibus, lineam circiter longis, confertis, flavescentibus v. in partibus junioribus flavovirentibus, e tuberculis parvis subviscidis ortis hirtos. Ramuli alterni, sesquipollicem dissiti, simplices, infimi pedales, superiores sensim breviores, omnes folii delapsi, e cujus axilla sunt enati stipulis, summi ipsis foliis stipati, dense setoso-hispidi. Folia alterna, sesquipollicem v. pollicem longa, abrupte pinnata, subvigintijuga, pctiolo filiformi-tereti, setulis brevibus hispidulo, basi nonnihil incrassato-dilatato, apice in acumen breve disinente. Foliola alterna, ovato-oblonga, infima quatuor lineas longa, una linea paullo latiora, superiora gradatim minora, basi rotundata, juxta superius latus petiolulo brevissimo complanato insidentia, hinc inaequilatera, latere inferiore convexo, basi nonnihil productiore, prope apicem sub lente subserrulato, latere superiore rectiusculo, inte- 
gerrimo, in mucronem brevem, rigidulum, rectum v. sursum nonnihil incurvum desinente, nervis pagina superiore obsoletis, inferiore manifestis, tribus v. quatuor, quorum unus margini superiori approximatus validior, setis raris, longiusculis ciliatus, reliqui crassitie subaequales, glabri, proximus cum superiore in mucronem apicis confluens, tertius intra marginem inferiorem desinens, quartus saepe nullus v. paullo supra basim in marginem solutus. Stipulae ad basim petioli geminae, acuminatolanceolatae, duas circiter lineas longae, basi lineam latae, scarioso-membranaceae, striato-multinerves, setis longis, rigidulis ciliatae, persistentes. Racemi in foliorum axillis solitarii, bi-decemflori, folio breviores, pedunculo patente, pedicellisque e basi crassiore superne attenuatis, apice inflexis, setosis. Bracteae ad basim pedicellorum scarioso-membranaceae, stipulis foliorum omnino conformes. Bracteolae in apice pedunculi calycis basim stipantes geminae, sessiles, lanceolatae, tres et dimidiam lineam longae, acutae, erectiusculae, rigidulae, nervosae, ad nervos extus pilis rigidulis, Iongioribus, intus multo brevioribus conspersae, margine rigide ciliatae, apice subserrulatae, foliaceo-virides. Calyx usque ad basim bipartito-bilabiatus, coriaceo-subscariosus, pallide fuscescens, striato-nervosus, inter nervos obsolete reticulatus. Labia a latere compressa, superius corollae vexillo incumbens, sex et dimidiam lineam longum, explanatum duas lineas latum, apice obtusum, sinu acuto plus minus profunde emarginatum, dorso acute convexum, ibique et prope basim angulo ad pedicellum fere recto incurvatum, pilis rigidulis, patentibus conspersum, margine subtilissime ciliatum. Labium inferius patentiuscule deflexum, septem lineas longum, tres et dimidiam lineam latum, basi ovatum, usque ad medium trifidum, laciniis lanceolatis, acutis, lateralibus planis, margine superiore rectiusculo, inferiore convexiore, intermedio acute carinato, vix quidquam longiore, omnibus dorso ad nervos pilis, ad margines ciliis rigidulis instructis, in setam brevissimam desinentibus. Corollae vexillum elliptico oblongum, novem lineas longum, tres lineas latum, basi sensim in unguem angustatum, complicatum, calycis labio superiori parallelum, apice obtuso, breviter exserto, adscendente, juxta totam longitudinem confertim striato-venosum, glaberrimum. Alae oblongae, obtusae, sex et dimidiam lineam longae, duas lineas latae, basi in unguem fere teretem, semilinearem abruptim angustatae, apice barbato-ciliatae, marginibus glabrae, caeterum nervosae, et inter nervos sub lente pulcherrime transversim plicato-rugosae. Carinae dipetalae petala subfalcata, septem lineas longa, margine superiore fere rectilinea, inferiore convexa, medio inter se cohaerentia, apice obtusissimo et basi superne in appendicem brevissimam, obtusam producta, et abruptim in unguem unam lineam longum transeunte inter se distincta. Stamina 10, monadelpha, tubo antice fisso, ima basi postice callo brevi aucto, sex lineas longo, filamentorum parte libera filiformi, antheris parvis, ovatis. Ovarium stipiti filiformi glaberrimo, sesquilineari impositun, duas et dimidiam lineam longum, compressiusculum, tenue, moniliforme, articulis $\eta$ v. 8, pilosum. Stylus filiformis, glaberrimus, quatuor lineas longus, incurvus; stigma antice truncatum. Legumen calyce inclusum, corolla staminibusque emarcidis stipatum, stipitatum, septem octoarticulatum, articulis secedentibus, semiovatis, dorso truncatis, coriaceo-membranaceis, obsolete pilosis, angustissime marginatis, monospermis. Semina spadicea, nitida, globoso-compressa. Embryonis radicula brevis, super cotyledonum crassissimorum commissuram prona, micropylen minimam, supra umbilicum foveolaeformem, margine angustissime elevato cinctum sitam attingens, vasorum chalazac reticulo immediatim infra umbilicum cordiformi. 
\%. Boronia ternata Endl. B. foliis sessilibus ternatis, foliolis obovatis cano-tomentosis, pedunculis axillaribus solitariis unifloris folio multo brevioribus.

In Novae-Hollandiae austro-occidentalis interioribus legit cl. Roë.

Rami rigidi, flexuosi, fastigiati, pube minutissima cinerei. Folia opposita, sessilia, ternata. Foliola sessilia, obovata, duas nonnisi lineas longa, unam lineam lata, obtusissima, basi angustata, utrinque tomento cinereo densissimo induta, inter se aequalia, v. intermedium nonnunquam paullo minus. Pedicelli in axillis foliorum solitarii, uniflori, vix quartam unius lineae partem longitudine aequantes, basi bracteolis duabus minutissimis stipati. Calycis quadripartiti laciniae ovatae, acutae, lineam longae, ut folia tomentosae. Corollae petala calyce duplo longiora, pallide rosea, pubescenti-tomentosa. Staminum filamenta infra apicem filiformem dilatata, alterna petalis opposita triente minora, antherae apiculatae. Capsulae pube stellata consitae, una alterave saepissime abortiva.

8. Grevillea Thelemanniana Hügel. Gr. foliis trifidopinnatifidis, laciniis linearibus subtus bisulcatis submucronatis, junioribus adpresse subpubescentibus, racemo denso.

Colitur in horto Hügeliano, e seminibus e Novae-Hollandiae ora austro-occidentali allatis enata.

Frutex elegans, in speciminibus cultis humanae altitudinis. Folia alterna, pollicaria $v$. sesquipollicaria, trifido-pinnatifida, lacinulae angustissime lineares, 6-8 lin. Iongae, vix dimidiam lineam latae, alternae v. oppositae, apice obtuso brevissime mucronatae, marginibus arcte revolutis et nervo subtus prominulo bisulcae, juniores pube adpressa rara conspersae. Racemus terminalis, ovatus, densus, pollicaris. Pedicelli juxta rhachim subverticillati, glaberrimi, duas lineas longi, divaricato-deflexi. Perigonium glaberrimum, rubicundum, 5 lin. Iongum, e basi ampliata sursum angustatum, hinc fissum, laciniis apice revolutis, staminiferis, margine ciliatis. Glandula hypogyna carnosa, lutea, truncata, erosodenticulata, mel copiosum secernens. Stipes ovarii duas lineas longus. Stylus 9 lin. Iongus, glaberrimus, rubicundus. Stigma oblique terminale. Ovula 2, ex apice loculi collateraliter pendula.

ANTHOTR0CHE Endlicher Gen. plant. Suppl. n. $\frac{3902}{1}$ Calyx quinquefidus. Corolla hypogyna, infundibuliformis, tubo sursum ampliato, limbi rotato-quinquelobi lobis obtusiusculis. Stamina 5, fertilia, corollae fauci inserta, exserta; filamenta subulata, fexuosa, antherae subbiloculares, loculis confluentibus, rimatransversali, unica apertis. Ovarium biloculare, placentis dissepimenti basi utrinque adnatis, multiovulatis, ovulis adscendentibus, anatropis. Stylus simplex; stigma capilatum, emarginatum. Capsula globosa, bilocularis, quadrivalvis, septo membranaceo, inferne medio placentifero, libero. Semina plurima, adscendentia, basi incurva, reticulata. Embryo in axi albuminis carnosi; cotyledoni- 
bus brevissimis, obtusis, radicula tereti, basi homotrope incurva, umbilico prope basim laterali parallele contigua. — Frutex NovaeHollandiae austro-occidentalis, densissime lanato-tomentosus, foliis alternis, crassis, junioribus axillaribus subfasciculatis, floribus in foliorum juniorum axillis solitariis, subsessilibus, parvis, violaceis.

\section{Anthotioche pannosa Endl.}

\section{In Novae-Hollandiac austro-occidentalis interioribus legit} cl. Roë.

Fruticis ramos video pedales, opposite ramulosos, lignosos, pennae anserinae tenerioris crassitie, tonento denso, sordide cinereo pannosos. Folia alterna, in inferiore ramulorum parte quinque circiter lineas dissita, in superiore magis magisque approximata, obovata v. obovato-rotundata, obtusissima, basi nonnihil attenuata, novem lineas longa, quatuor et dimidian lata, integerima, crassiuscula, tomento denso induta. Ramuli axillares, foliis breviores, rarissime aequilongi, foliis junioribus, dense confertis, tres lineas longis, duas latis, tomento pallide stramineo vestitis velati. Flores in inferioribus foliorum ramulos axillares vestientium axillis solitarii, sessiles. Calyx quinquefidus, laciniae lineari-lanceolatae, duas lineas longae, dimidiam lineam latae, extus densissime lanatae, intus glabrae, uninerves, reticula to-venosae, erectae. Corolla hypogyna, tubo calycem aequante, sursum ampliato, limbo tubuin aequante, rotato-explanato, ad medium quinquelobo, violaceo, striato, lobis obtusiusculis, utrinque imprimis extue lanato-pubescentibus. Stamina 5, omnia fertilia, corollae fauci inserta, limbum aequantia ; flamenta basi nonnihil dilatata, subulata, subflexuosa, inferne pubescentia, villorum fascicalis ab eorundem origine ad basim tubi decurrentibus, antherae biloculares, loculis ovatis, supra connectirum divaricatis, apice confluentibus, rima unica transversa apertis, post pollinis emissionem explanatae, suborbiculares, disco villoso. Ovarium ovatum, biloculare. Ovula juxta placentas dissepimenti basi utrinque insertas plurima, adscendentia, anatropa. Stylus terminalis, filiformis, flexuosus, glaber, lineam longus. Stigma obtusum. Capsula calyce recondita, diu corollae reliquiis etiam stipita, lata basi sessilis, sulglobosa, Pisi minoris mole, bilocularis, quadrivalvis, valvis memJranaceis, reflexis, demum explanatis, septo membranaceo libero, basi medio utrinque in placentam, in nervum usque ad septi apicem excurrentem desinentem inerassato, caeteruı tenero; fragili. Semina plura, oblonga, fnscescentia, scrobiculata, adscendentia, prope basim incurva, et paullo supra basim obsolete umbilicata. Embryo in axi albuminis carnosi rectiusculus, subcylindricus; cotyledonibus brevissimis, obtusis, radicula tereti, basi nonnihil incurva, umbilico parallele contigua.

DIDYMANTHUS Endlicher Gen. plant. Suppl. n. $\frac{1920}{2}$ Flores unisexuales Masc. ..... Fem. axillares gemini, patentes, basi connati. Perigonium tubo subcylindrico, fauce squamulis 5 clausa, limbi quinquepartili lobis venosis, demum auctis, scariosis. Filamenta 5, hypogyna. Ovarium ovalum, uniloculare, uniovulatum. Stylus bipartitus. Utriculus membranaceus, perigonii tubo indurato inclusus. Semenverticale, compressum, testa membranacea. Embryo periphericus, albumen farinaceum cingens, radicula supera. - Fruticulus Novae-Hollandiae austro-occidentalis, hu- 
milis, ramosus; foliis anguste lanceolatis, piloso-canis, apice recurvis, floribus axillaribus, ebracteatis.

\section{Didymanthus Ihoëi Endl.}

\section{In Novae - Hollandiae austro - occidentalis colonia Swan-River.} legi६ cl. Roë.

Specimen unicum est fruticulus quadripollicaris, radice tenui, elongata, flexuosa, ramulis fibrillisque paucis aucta. Caulis strictiusculus, tenellus, cortice cinerascente, rugoso, sparsim piloso vestitus, foliorum delapsorum cicatricibus notatus, ramulis aliquot accessoriis, e foliorum jam delapsorum axillis prodeuntibus, abbreviatis, confertim foliosis auctus, apice in ramos aliquot approximatos, sesquipollicares, horizontaliter patentes, pilis adpressis, candicantibus dense sericeos divisus. Folia confertim alterna, auguste lanceolata, duas lineas longa, dimidiam lineam latitudine parum superāntia, acuta, basi adpressa, apice subrecurva, utrinque pilis albis, adpressis, imprimis in junioribus confertis, subsericeis canescentia. Flores masculi non visi. Feminei in axillis foliorum gemini, ad angulum rectum dirergentes, et ipsis basibus intime inter se connati. Perigonii tubus subcylindricus, sesquilineam longus, extus adpresse pilosus, intıs laevigatus, sursum nonnihil angustatus, fructifer quidquain auctus et induratus, faux obsessa squamulis quinque, late triangularibus, quadrantem lineae longis, pilosulis, limbi lobis oppositis, conniventibus, in fructu faucem claudentibuș. Limbi quinquepartiti lobi obovato - cuneati, unam lineam longi, utrinque pilosuli, obtusissimi, subcolorati, sub lente flabellatim venosi, patentes, frutiferi aucti, scariosi, rotato-patentes. Filamenta hypogyna (stamina sterilia?) quinque, linearia, acuta, hyalina, tubum perigonii subaequantia. Ovarium uniloculare, sessile, fere obconicum, tenuissime inembranaceum, membrana apice incrassata quasi duplicata, operculi speciem exhibens. Ovulum unicum, ex apice fili tenuissitui, ovarii parieti hinc adpressi, revera lißeri pendulum. Stylus terminalis, brevissime exsertus $\mathbf{v}$. faucem aequans, bipartitus $\mathbf{v}$. rarissime tripartitus, cruribus filiformibus, papilloso-stigmatosis, duobus aequalibus, tertio dum adest multo breviore. Perigonia fructifera angulo obtusissino inter se connata, basibus in massam solidam suberosam, tres quartas lineae partes !metientem confluentibus, tubo indurato, vix aucto, fauce squamulis quinque arcte conniventibus reclusa, limbi quinquepar. titi lobis scarioso-membranaceis, bilinearibus, rotato-patentibus, parce pilosis, flabellatim subvenosis, margine obsolete undulatis. Utriculus intra perigonii tubum liber, $3 / 4$ lin. longus, ovatus, tenuissine membranaceus, fuscescens, inaequilaterus, apice hinc seminis extresnitate radiculari protuberante rostratus, illinc declivis, pericarpii membrana duplicata, operculiformi, excentrice styligera oblique tectus. Semen verticale, compressun, integumento tenuissime membranaceo. Embryo viridis, periphericus, albumen farinaceum parcum cingens; cotytedonibus oblongo-linearibus, radicula cylindrica, supera. 
Nr. 2.

\title{
NOVARUII STIRPIUII
}

\section{DECADES}

\section{Editae a Museo Caesareo palatino Vindolbonensi.}

\author{
Nr. 2. Vindobonae, typis Sollingerianis. 15. Maji 1839.
}

ONCOSPORUM Putterlick. Calyx minimus, quinquepartitus, laciniis aequalibus. Corollae petala 5, hypogyna, aequalia, subspathulata, patentia, apice reflexa. Stamina 5 , hypogyna, aequalia ; filamenta filiformia, apice flexa, antherae biloculares, longitudinaliter dehiscentes, tandem reclinatae. Ovarium rectum, elongato-ellipsoideum, biloculare. Ovula in loculis plurima, juxta axim dissepimenti utrinque biseriata, horiæontalia, anatropa. Stylus filiformis, ovario continuus; stigmate simplici, hemisphaerico terminatus. Fructus capsularis, stylo persistente superatus. Capsula ovoidea, utrinque compressa, membranacea, bilocularis, loculicide ab apice ad medium dehiscens, semivalvata, mox septifrage complete bivalvis, semiseptis in axi contiguis tandem hiantibus, margine cavum spectante seminiferis. Semina plura, globosa; rhaphe ab umbilico ad chala $\approx a m$ intra testam libera, tumida, discolor. Embryo in basi albuminis cornei prope umbilicum minimus, orthotropus. - Suffrutex Novae-Hollandiae austro-occidentalis; ramis volubilibus, foliis alternis, argute serratis, pedunculis oppositifoliis, floribus candidis, purpureo striatis.

Genus hinc Mariantho Endl., illine Bursariae Cav, affine.

\section{Oneospormm bicolor Putterlick.}

\section{Nova-Hollandia austro-occidenialis. (Hügel.)}

Frutex tripedalis et ultra. Radix teres, deorsum sensim attenuata, recta, lurida, ramulis fibrillisque paucis aucta. Truncus cylindricus, semipedalis, pennae anserinae crassitie, erectus, hinc inde flexus, cortice fusco, rugoso vestitus, ramisque primariis ternis quaternisve terminatus. Rami teretes, superne sensim attenuati, erecto-patentes, primum recti, tandem flexuosi et volubiles, cortice badio, glabro vestiti. Ramuli ex axillis foliorum surgentes caeterum conformes. Folia trunci primaria bina opposita, plana, tenuia, circumscriptione obovata, ad dimidium pollicem longa, 2 lineas lata, basi sensim in petiolum angustata, apice obtusata, margine integerrima, sequentia solitaria, remota, in seriem truncum, ramos ramulosque spiraliter circumeuntem disposita, obovata vel obovatoelliptica, ad sesquipollicem longa, semipollicem lata, argute serrata, imo 
suprema fimbriato dentata, patentia, flavo-virentia, juniora utrinque pilis brevibus, ad nervum subtus prominentem confertioribus consita, superiora glabra. Pedunculi in ramis oppositifolii, solitarii, uniflori, erecto patentes, teretiusculi, ad 3 lineas longi, filiformes, setis raris rigidis conspersi, basi bractea squamaeformi decidua, vix quadrantem lineae aequante stipati, paullo supra basim pedicellis duobus antrorsum sublateralibus, bracteola quoque vix conspicua basi stipatis, et cicatricibus duabus notatis instructi, superius cicatrice una alterave ornati, in receptaculum floris sensim incrassati, sub anthesi nutantes. Calycis foliola 5 , inter se aequalia, aestivatione imbricata, ovata, acuta, sesquilineam longa, badia, glaberrima, corollae alpressa, demum decidua. Corollae petala 5, hypogyna, aequalia, calycis foliolis alterna, aestivatione marginibus sese imbricatim tegentia, sub anthesi patentia, apice reflexa, subspathulata, $5^{\prime \prime \prime}$ longa, ad sesquilineam lata, apiculata, ungue basi glandula luteola instructo, candida, venis longiludinalibus punctisque intermediis purpureis ornata. Stamina 5, hypogyna, aequalia, petalis alterna et breviora. Filamenta filiformia, in tubum incurvum conniventia, stricta, post anthesin patentia, sursum flexa. Antherae terminales, ovatae, obtusae, sesquilineam metientes, basi emarginata filamentis affixae, purpureae, erectae, post anthesin retrorsae, illoculares, loculis oppositis, inferne contiguis, connectivo adnatis, superne subdivergentibus, longitudinaliter introrsum dehiscentibus. Pollinis lutei granula globosa, triedra. Ovarium basi glandulis quinque cum antheris alternantibus cinctum, elongato-ellipsoideum, compressiusculum, glabrum, venulis obscurioribus notatum, superne in stylum teretem, filiformem, stamina vix superantem, cavitate quidquain breviorem continue transiens, intus biloculare, loculis multiovulatis. Stigma in apice styli simplex, hemisphaericum, papillosum. Ovula secundum axim dissepimenti utrinque biseriata, bina invicem opposita, funiculo brevi, introrsum inclinato suspensa, subglobosa, horizontalia, micropyle ad latus externum prope umbilicum aperta. Fructus e receptaculo floris superstite brevi suffultus, capsularis, stylo brevissimo persistente superatus. Capsula ovoidea, utrinque compressiuscula, faciebus planioribus sutura longitudinali impressa sulcatis, coloris fulvi, venis saturatiooribus varie anastomosantibus percursa, glabra, membranacea, bilocularis, maturitate loculicide ab apice ad medium semivalvata, mox septifrage complete bivalvis, semiseptis in axi contiguis tunc hiantibus, margine cavum spectante seminiferis. Semina funiculo vix $1 / 4^{\prime \prime \prime}$ longo corrugato suspensa, globosa, lineam metientia, horizontalia, laevia, nitida. Rhaphe axim capsulae versus horizontaliter per quadrantem circumferentiae seminis producta, ab umbilico ad medietatem libera, in torum tumida, adhaerens; integumento communi, ibidem coloris sanguinei, laevi, nitido, sat duro vestita, intus inanis, tubulo nonnisi membranaceo pellucido, tenero, fasciculuın vasorum in filum tenuissimum corrugatum continente, et ad chalazam in placentulam orbicularem vasis pertextam desinente, percursa. Integumentum commune sive testa e strato cellularum simplici coloris profunde umbrini, tenera, opaca, sat dura, adnata strato cellularum teneriori, transparenti, pallidi coloris, cum membrana tubuli rhaphes continuo, in regione chalazae placentulam vasculosam includente, nucleum ipsuin undique arcte ambiente. Nucleus membrana tenerrima, transparente, tandem a chalaza soluta vestitus. Albumen subglobosum, ad chalazam complanatum, integrum, solidum, corneum, coloris lactei, in laminis tenerrimis transparens, ad micropylem impressum, ibique cavitatem ellipsoideam, clausam, humore repletam, vix $\frac{1}{200}$ albuminis partem consumentem, et embryonem undique includentem continens. Embryo in fundo cavitatis situs, sacculo proprio tenerrimo pellucido inclusus, vix ${ }_{6}^{1}-0$ lineam permetiens, basi attenuata umbilicum spectans, apice obtuso paullulum emarginatus, coloris lactei, transparens, simplex, cellulosus, partibus certis nondum discermendis. 
DASYMALLA Endlicher Gen. plant. Supplem. n. $\frac{3733}{1}$. Calyx quinquepartitus, fructifer vix auctus. Corolla hypogyna, infundibuliformis, tubo calycem superante, fauce aequali, limbi bilabiati labio superiore bilobo, inferiore trifido subaequilongo. Stamina 4, imo corollae tubo inserta, inchsa, subdidynama. Ovarium incomplete quadriloculare, septis incompletis, loculis uniovulatis. Ovula pendula, anatropa. Stylus terminalis; stigma acutum, bifidum. Drupa baccata, putamine quadriloculari, tetraspermo. Semina inversa. Embryo.... - Fruticuli Novac-Hollandiae austro-occidentalis, dense lanati ; foliis oppositis, oblongo-obovatis, integerrimis, pedicellis axillaribus fasciculatis, v. in cymas oppositas in racemum terminalem dispositas collectis, foribus purpurascentibus, corollae tubo basi barbato, fauce nuda.

Genus Myoporineum, vel habitu facillime distinguendum.

12. Dasymalla axillaris Endl. D. floccoso-lanata, pedicellis axillaribus fasciculatis folio brevioribus.

\section{In Novae-Hollandiae austro-occidentalis interioribus legic} cl. Rö̈.

Fruticulus pedalis, multicaulis, a basi opposite ramosus. Rami tetragoni, fere pennae anserinae crassitie, lana alba dense vestiti. Folia opposita, paribus inferioribus remotis, supremis approximatis, obovatooblonga, pollicem v. sesquipollicem longa, quatuor v. quinque lineas lata, integerrima, obtusa, basi angustata, densissime albo-lanata, lana ad margines longiore subfloccosa, scalpello derasa sub indumento rugosa, nervo medio prominente. Pedicelli in axillis foliorum 3-4 congesti, filiformes, quatuor lineas longi, rigiduli, antice et postice lana patente plumosi, margine utroque glabriusculi, basi utrinque bracteola linearisetacea, dense lanata, mox obliterata stipati. Calyx ad basim quinquepartitus, laciniis subaequalibus, subcampanulato-conniventibus, linearibus, obtusis, quatuor lineas longis, tres quartas unius lineae partes latitudine vix superantibus, extus densissime lanatis, lana ad margines longiore, patente subplumosis, intus nudis, trinerviis, nervis lateralibus marginantibus. Corollae tubus quinque et dimidiam lineam longus, intus sequilineae supra basim altitudine annulo staminigero densissime piloso munitus, sursum ad medium ampliatus, ex sicco inferne flavescens, superne cum limbo purpureus, limbus bilabiatus, labiis subaequilongis, superiore bilobo, inferiore trifido, lobis obtusis, circiter tres lineas longis, labii inferioris lobo medio panllo longiore. Stamina 4 , subdidynama, corollae faucem aequantia; filamenta filiformia, glaberrima, antherae bilocnlares, dorso insertae, apice obtusae, basi bifidae. Ovarium compresso conicum, dense pilosum, carpidiorum marginibus introflexis, bilamellatis, ad axin hand pertingentibus prima juventute uniloculare, mox ad axim connatis, marginibusque revolutis parietem oppositam attingentibus quadriloculare. Ovula in loculis solitaria, ex angulo pendula, anatropa. S'ylus terminalis, filiformis, staminum longitudine, apice breviter bifidus, lobis acutis. Drupa baccata, putamine quadriloculari, tetraspermo. Scmina inversa. 
13. Dasymalla terminalis Endl. D. tomentoso-lanata, cymis oppositis in racemum terminalem dispositis.

\section{In Novae-Hollandiae austro-occidentalis interioribus legit cl. Rö̈.}

Rami teretiusculi, tomento denso albo induti. Folia opposita, obovato-oblonga, decem lineas longa, quatuor lineas lata, dense tomentosa, in apicibus ramulorum conferta, floralia paullo minora, minus lanata, paribus tribus ad quinque, internodiis uni-bipollicaribus, minus dense lanatis sejunctis. Cymae in axillis foliorum floralium oppositae, simplices, iisdem breviores. Flores quam in praecedente specie paullo minores, $c a$ lycis parcius lanati laciniis lanceolatis.

14. Trlbachia Bonplandiana Fenzl. I. annua, glaberrima, caule simplici erecto, foliis late ovatis acutis $\left(1 / 2-2^{\prime \prime} \lg .\right)$ petiolatis, cymae semel dichotomae ramis elongato-racemiformibus flexuosis, floribus demum nutantibus subsecundis, calyce pedicellis duplo, capsula ellipsoidea subtriplo breviore, corollae infundibuliformis albae ( $1 / 2$ " lg.) laciniis ovatis extus glandulosis, staminibus inaequalibus inclusis, styli bicruris stigmate, simplicissimo.

Synon. Claytonia nemorosa Willd. herb. et msc. in R. et Schult. Syst. V. 436. - Bonpland msc. in Kunth Nov. gen. am. VI. 80.-DC. Prodr. III. 362.

Patria. America aequatorialis. Habitat in umbrosis Javitae, missionis Orinoqueensis, reipublicae Novo-Granadensis $-3^{0} l$.bor. (Bonpland!). - V. sp. unicum a cl. Humboldt commun. in herb. Willd.!

Herba annua, glaberrima, caule speciminis visi simplicissimo, stricte erecto, quadrangulari, cum inflorescentia spithameo, filo emporetioc mediocri haud crassiore, internodiis 5 inaequilongis, medio majore $1 \frac{1}{2}$ unciali absoluto. Folia penninervia, late ovata, acuta, integerrima, in petiolum lamina $1 / 2-4$ plo breviorem, angustum, basi rursus cuneato - attenuata, ad noduna decurrentem in cupulam brevissimam adpressam connata, patentia; inferiora cum petiolo $1 / 2-1^{\prime \prime}$, reliqua $1 \frac{1 / 2}{2}-2^{\prime \prime}$ longa, minora 4-8"', majora $1^{\prime \prime}$ et parum ultra lata; aqua emollita odorem levem herbae Nicotianae siccatae spirantia, masticata vix amaricantia. Cy$m a$ semel bifurcata terminalis, pedunculo semiunciali parum longiore suffulta, ramis erecto patulis, rigidulis, a flore ad florem flexuosis, racemiformibus, digitalibus, aphyllis; floribus per internodia, inferius 3-6 $6^{\prime \prime \prime}$ longa, rhythmo certo ulterius decrescentia remotis, cujusvis racemi $6-8$, pedicello primum erecto, demum cernuo, calyceque duplo longiore, bracteola scariosa obsoleta, late subulata, ad pressissima basi lateraliter stipato insidentibus. Calyx turbinatus, demum $1^{1} /^{\prime \prime \prime}$ longus, usque ad basim quinquepartitus, intus corona papillarum subtilissimarum biseriali, sinubus inserta barbatus, laciniis ovatis, acutiusculis, in alabastro obsolete imbricatis, nec strenue valvatis, in fructu capsulae adpressis. Corolla infundibuliformis, semiuncialis, alba (fide Bonpl.), in sicco dilute ochracea, fauce infundibulari, lobos ovatos, obtusiusculos, $2^{\prime \prime \prime}$ longos, extus marginibusque glandulis substipitatis, sub lente solum conspicuis, dense vestitos aequante, in tubum calyce parum longiorem, supra basim constrictum, ibidemque circumscisse demum rumpentem sensim angustato, decidua Stamina 5 , subinaequilonga, glabra, mediae fauci inserta, corol- 
lac laciniis breviora. Antherae lineari-oblongae, loculis virginalibus appositis, parallelis, connectivo apice in mucronulum producto supra medium adnatis, erectae, effoetae demum recurvae, loculis contortis, basi sagittatodiscretis. Pollinis granula simplicia, echinata. Ovarium biloculare, multiovulatum, carpophyllorum faciebus introflexis tota longitudine coalitis, intra loculos in laminam placentiferam indivisam $v$. nargine subbifidam productis; stylo bicruri, stigmate simplicissimo acuto terminatum. Ovula amphitropa, sessilia. Capsula ellipsoidea, chartacea, calyce triplo longior, septicide bivalve dehiscens, valvulis follicularibus, styli persistentis cruribus apice firmius cohaerentibus diutius connexis, demum liberis, tota longitudine faciei commissuralis laminarum placentiferarum discessu apertis, navicularibus, rostratis. Semina plnrima, minima, 11/6"' lata, varie compressa, cubica, faciebus medio depressa, quasi umbonata, peltatinı affixa, testa dilute testacea, membranacea, cellulari, rugulosa, firmissime adhaerente tunicata, exalata. Albumen corneum, copiosum, coctione granulosum, carnosum. Embryo axilis, minimus, punctiformis, globosus, radicula et cotyledonibus vix distinctis.

15. Dillwymia elegang Endl. D. ramulis adpresse sericeis subcanescentibus, foliis teretiusculis canaliculatis laevibus erectopatentibus, mucrone brevissimo recto, floribus axillaribus solitariis geminisque racemosis, pedicellis calycis tubum subaequantibus.

\section{Colitur in horto Hügeliano.}

Species elegans, D. floribundae et $D$. sericeae affinis. Ramuli tenelli, pube adpressa, sericea subcanescentes. Folia sparsa vo sapissime conferta, et nonnunquam ternatim approximata, erecto-patentiuscula, teretiuscula, octo lineas circiter longa, dorso convexa, facie sulcata, utrinque saturate viridia, tuberculis minimis, nonnisi sub lente conspicuis, raris instructa, pilis brevibus, candicantibus, adpressis prima juventute hinc inde conspersa, demum glabrata, apice in mucronem brevissimum, rectum desinentia, ima basi attenuata, ibique decolora, quasi hyalina. Pedicelli in inferiorum foliorum axillis solitarii, in superiorum saepe gemini, sesquilineam longi, basi bracteis duabus linearibus, subscariosis, obtusis, vix dimidiam lineam longis stipati, altera $v$. interdum utraque bractea pedicelli alterius rudimentum plus minus manifestum fovente. Bracteolae 2 , medio pedicello insertae, bracteis conformes, acutae. Calyx campanulatus, glaberrimus, tubo sesquilineari, basi attenuato, limbi bilabiati, lineae longitudinem paullo superantis labio superiore duas fere lineas lato, emarginato-bilobo, lobis divaricatis, acutiusculis, binerviis, inferioris tripartiti lobis triangularibus, acutis, uninerviis, patentiusculis. Corollae vexillum septem lineas latum, emarginatum, luteum, lamina ut in plerisque congenerum picta, ungue lato, canaliculato, duas lineas longo. Alae tres et dimidiam lineam longae. Carina trilinearis, obtusa. Stamina congenerum, calycis tubo paulio infra medium inserta. Ovarium sericeum.

16. Dillwynia Pufolia Endl. D. ramulis glabris, foliis filiformi-teretibus erecto - palentibus laevibus glaberrimis muticis v. submucronatis, corymbo terminali longe pedunculato dense plurifloro. 
Species ab affinibus, $D$. pedunculari Benth., $D$. parvifolia $R, B r$, et $D$. ramosissima Benth. facilline distinguenda: partium omnium glabritie et foliis multo longioribus. Ramuli tenerrimi, glabri. Folia sparsa, filiformisemiteretia, dimidium pollicem longa, subcanaliculata, subtus convexa, glaberrima, erecto-patentia, recta nec unquam torta, apice nonnihil recurva, mutica v. mucrone brevissimo, rectol, innocuo, laevia, glaberrima. Pedunculi ramulos terminantes sex ad octo lineas longi, stricti, glaberrimi, apice flores $6-10$ in corymbum aggregatos gerentes. Pedicclli unam lineam longi, basi bractea subulata, parva, supra medium bracteolis duabus lineari-lanceolatis minutis muniti. Caly $x$ campanulatus, glaberrimus, tubo sesquilineam longo, linbo unam lineam metiente, labii superioris late hilobi lobis divergentibus, obtusiusculis, inferioris tripartiti laciniis late triangnlaribus, acutioribus. Corollae petala paullo infra medium calycis tubum inserta, decidua; vexillum ungue bilineari, late semiorbiculatum, emarginatum, duas lineas longum, tres et dimidiam lineam latum, pallide flavum, basi macula purpurascente; alae oblongae, obtusissimae, subfalcatac, tres lineas longae; carina recta, obtusa, vix duas lineas longa, pallida. Stamina decem, libera, glabra, caduca. Ovarium oblongum, compressum, brevissime stipitatum, adpresse sericeum, biovulatum. Stylus filiformis, uncinatus, glaberrimus. Stigma terminale, minutissime capitatum.

1\%. Dillwynia seroiphioides Endl. D. ramulis hispidulis, foliis erectiusculis tortis laevibus glaberrimis, mucrone brevissimo recto v. incurvo, corymbis terminalibus sessilibus paucifloris, calycibus hispidulis.

\section{Colitur in horto Hüyeliano.}

Proxima Dillwyniae phylicoidi Cunningh. optime distincta : foliis longioribus, semper valde tortis, glaberrimis, laevibus, floribus majoribus. Ramuli tenelli, purpurascentes, parce hispiduli. Folia sparsa, linearisubulata, quatuor v. quinque lineas longa, erecto-patentia, marginibus involuta, omnia dextrorsum torta, laevia, glaberrima, saturate viridia, apice obtusiuscula $\mathbf{v}$. in mucronem brevissimum, subrecurvum desinentia, juniora nonnunquam pone mucronem setulis aliquot brevibus, mox defluentibus munita, basi in petioli brevissimi hyalini speciem attenuata. Corymbi in apicibus ramulorum e supremorum foliorum axillis conferti, triquadriflori, sessiles. Pedicelli basi bractea squamaeformi minima stipati, unam lineam longi, hispiduli, medio bibracteolati, bracteolis squamaeformibus, lineari-lanceolatis, obtusis, dimidiam lineam longis. Calyx campanulatus, tres lineas longus, hispidulus, bilabiatus, labii superioris late bilobi lobis divergentibus, inferioris tripartiti laciniis late lanceolatis. Corollae petala disco medium calycis tubum vestienti inserta. Vexillum unguiculatum, ungue lato, bilineari, lamina patente, duas lineas longa, quinque et dimidiam lata, emarginata, lutea, basi sanguineo picta. Alac oblongae, obtusissimae, rectae, tres lineas longae, unam lineam latae, luteae, carinam obtusam, parum incurvam, duas et dimidiam linean longam, pallide viridem, solo apice sordide purpuream conniventim tegentes. Stamina libera, glabra, caduca. Ovarium brevissine stipitatum, oblongum, adpresse sericeum, biovulatum. Stylus crassiusculus, glaber, apice uncinatus; stigmate capitato. 
18. Daviesia macroplnylia Endl. D. foliis late oblongis planis coriaceis basi angustatis apice obtusis muticis, racemis axillaribus solitariis v. geminis folio brevioribus basi multibracteatis apice corymbiferis, pedicellis calycem aequantibus.

\section{Colitur in horto Hügeliano.}

Species D. corymbosae Smith affinis, foliorum forma et magnitudine, pedicellisque calycem haud superantibus distinctissima. Caulis inferne angulatus, rami teretiusculi. Folia in inferiore caulis parte ternatim approximata, in ramis alterna, quinque pollices longa, sesquipollicem lata, coriacea, subfiaccida, glaberrima, basi angustata, apice obtuso mutica, uninervia, laxe venosa. Racemi in foliorum axillis solitarii v. saepissime gemini, dimidio v. triente breviores, basi bracteolis alternis dissitis, sterilibus, apice confertis corymbiferis instructi. Bracteolac submembranaceae, ovatae, acutae, unam lineam longae, glabrae, patentes, apice purpurascentes. Pedicelli bilineares, teretes, giaberrimi, sub calyce nonnihil constricti. Calyx glaberrimus, maculis purpureis conspersus, campanulatus, labio superiore dorso convexo, subtruncato, emarginato, inferiore aequaliter tridentato. Corollae vexillum ungue basi incurvo, una linea paullo breviore, lamina duas lineas longa, tres lineas lata, subemarginata, lutea, basi macula semicirculari, sordide purpureo-fusca. Alae oblongae, obtusae, fusco-purpureae, apice lutescentes, carinam fornicatam, obtusam superantes. Ovarium oblongum, glaberrimum, prope basim biovulatum. Stylus filiformis, incurvus, glaberrimus.

19. Teuconogon conifolins Endl. L. foliis lanceolatis acutis margine revolıtis patentibus v. deflexis ramulisque hispidis, spicis terminalibus paucifloris congesto-capitalis, calycibus bracteisque herbaceis villosis.

\section{Colitur in horto Hügeliano, e seminibus ad Swan-River col- lectis enata.}

Ramuli graciles, patentim hispidi. Folia patentia v. deflexa, lanceolata, ramulorum floriferorum sesquilinearia, sterilium paullo longiora, basi dimidiam lineam lata, acuta, mutica $v$. in setam brevem mollem desinentia, marginibus revoluta, subtus obsolete uninervia, utrinque hispida, adulta supra saepius glabrata. Flores in apicibus ramulorum $5-6$, capitato-congesti. Bracteae subtendentes herbaceae, ovatae, obtusissimae, dimidia linea paullo longiores, concavae, dorso villosae, marginibus albo-lanatae, intus glabrac. Bructeolae calyci adpressae, ovato-lanceolatae, acutae, calycis foliolis dimidio breviores, margine submembranaceae, villosae. Calycis foliola lanceolata, acuminata, lineam longa, extus villosa, marginibus lanata. Corollae tubus calycem aequans, limbi laciniae tubo aequilongae, lineari lanceolatae, marginibus revolutae, dense barbatae. 
20. Glossodia Hrumonis Endl. Gl. appendicis bipartitae lobis divergentibus truncatis, columna supra antheram producta.

\section{Habitat in Novae-Hollandiae austro-occidentalis colonia Swan- River. (Hügel.)}

Species insignis, propter columnam supra antheram productam in genere anomala, et in propria sectioue (Elythranthera) collocanda. Bulbus ovatus, quinque lineas longus, integumento lamelloso, sordide cinereo vestitus, apice in caudicem hypogaeum, sesquipollicarem, subtetragonum, pennae corvinae crassitie, sordide stupposum, rarissime hinc inde fibrilliferum continuus. Folium radicale unicum, elliptico-lanceolatum, sesquipollicem longum, duas lineas latum, obtusiusculum, utraque pagina pilis raris, nitentibus, longiusculis, mollibus, in paginae a caule aversae parte inferiore copiosioribus conspersum, margine obsolete ciliatum, basi vagina membranacea laxa, truncata, glaberrima, nitidula, duas et dimidiam lineam longa inclusum. Caulis scapiformis, uniflorus, quadriquinquepollicaris, tetragonus, tener, dimidiam lineam diametro vix superans, medio folio unico, quadrilineari, obtuso vaginatim amplexus, apice florali, vix quidquam majore, glanduloso-pubescente vestitus; pili in inferiore caulis parte rari, sericei, patentes, dnas et dimidiam lineam longi, supra folium pubes rara, brevis, simplex, pone folium florale confertior, glandulosa, capitulis minimis nigricantibus pilos singulos terminantibus. Pedunculus filiformis, strictus, folii floralis longitudine, glanduloso-pubescens. Ovarium cylindricum, inferne attenuatum, obsolete striatum, glanduloso-pubescens. Perigonii foliola 5, aequalia, ovata, acutiuscula, basi nonnihil angustata, qninque lineas longa, duas et dimidiam lineam lata, patentia, caerulea, subseptemnervia, apice nonnihil incrassata, maculis suborbicularibus saturatioribus picta, nitida, extus ad nervos sub lente pube glandulosa conspersa, ad margines paullo confertius ciliata. Labellum nanum, ovato-lanceolatum, obtusum, duas lineas longum, maxima baseos latitudine vix dimidiam lineam excedens, planum, glaberrimum, ex sicco pallide flavum, apice inflexo stramineo. Appendix inter labellum et columnain usque ad basim bipartita, fusca, lobis duas lineas longis, e basi ovata superne angustatis, apice truncatis, adscendentim divergentibus. Columna late et tenerrime membranacea, tres et dimidiam lineam longa, sesquilineam lata, $3 / 4$ lin. Longitudine ultra antheram producta, supra eandem fornicato connivens, obtusa, materie stylinea per mediam columnam procurrente lineari, autice canaliculata, infra antheram abrupte in gynisum transversim oblongo-quadratum desinente. Anthera ovato-acuminata, dimidiam lineam longa, loculis approximatis. Pollinia in quovis loculo gemina, compresso - subclavata, pulverea, basibus brevissime connexa, alterum minus. 
Nr. 3.

\section{NOVARUII STIRPIUII}

\section{DECADES}

\section{Elitac a Pruseo Caesareo palatino Vandobonensí.}

Nr. 3. Vindobonae, typis Sollingerianis. 20. Maji 1839.

FOCKEA Endlicher Gen. piant. Suppl. n. $\frac{3504}{1}$. Calyx quinquefidus. Corolla tubo brevi, linbo quinquepartito, patence. Corona staminea summo filamentorum tubo inserta, basi corollae postice adnala, decemfida, laciniis alternis ancheris oppositis tripartilis, lobo intermedio majore, basi intus processu recto aucto, alternis nanis, indivisis. Antherae appendice membranacea terminatae. Pollinia basi affixa, incumbentia. Stigma depresso-conicum. Folliculi ..... - Frucex capensis; caudice epigaeo tuberiformi, subgloboso, rugoso, ramis e caudicis apice simplicibus, subvirgatis, foliis oppositis, sessilibus, ovatis, cuspidatis, undulatis, pilis brevibus subcanescentibus, umbellis extraaxillaribus subsessilibus, tri-quinquefloris, floribus albidis.

Dixi in honorem Gustavi Woldemari Focke, Bremani M. D. micrologi et zootomi egregii, inter botanicos commentatione ${ }_{2} D e$ respiratione vegelabilium, Heidelbergae 1833. 4." celebris.

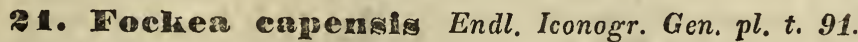

Habilat in Capite bonae spei, unde olim in hortum Schönorunnénsem illatam, post longam inertiam elapsis annis laete florentem humanissime demonstravic cl. Henricus Schott.

Caudex epigacus tuberiformis, in stirpe culta ovato-subglobosus, capitis humani mole, cortice rugoso-subtoroso vestitus. Rami ex apice caudicis simplices, subvirgati, pedales bipedalesque, fili emporetici crassitie, teretes, superne sensim attenuati, fuscescenti-rufi, pilis brevibus consiti, abscissi, uti et omnes reliquac partes ubi avelluntur, lac copiosum, spissum plorantes. Folia opposita, per internodia duos ad tres et dimidium pollicem longa sejuncta, sessilia, subcarnosa, rigida, circumscriptione ovata, basi abrupte angustata, apice brevissime cuspidata, marginibus undulato-subplicata, tres ad octo lineas longa, tres ad quinque lineas lata, utrinque pilis brevibus, ad nervum medium subtus valide prominentem confertioribus subcanescentia. Umbellae interpetiolares tri-quinqueflorae, subsessiles. Pedicelli teretiusculi, albidi, lineam longi, basi bractea squamaeformi, acuta, vix dimidiam lineam aequante stipati. $C a-$ $l_{y} x$ albus, corollae concolor, sesquilineam longus, tubo brevissimo, limbi 
quinquefidi laciniis lancenlatis, acutis, corollae tubo adpressis, pilis hrevibus conspersus. Corolla hypogyna, tnbo calycem paullo superante, vix bilineari, superne nonnihil anpliato, limbi quinquepartiti laciniae anguste lanceolatae, acutae, tres et dimidiam lineam longae, marginibus revolutae, rotato-patentes. Stamina corollae tubo supra basin inserta, in tubum connata. Corona staminea summo filamentorum tubo imposita, corollae faucem cingens, basi postice ejusdem tubo adnata, spatium inter corollae tubi parietem interiorem, staminumque tubi parietem exteriorem, pilis grumosis plenum claudens, e foliolis decem inter se coalitis composita; foliolis alternis antheris oppositis tripartitis, lacinia media duplo Iongiore, tereti, acuta, lateralibus planis, margine interiore deorsum producto, libero, introflexo, alternis corollae laciniis oppositis, lateri priorum alhaerentibus, indivisis, nanis, obtusiusculis, apice subreflexis. Processus corniformis, coronae laciniis unajoribus oppositus, eorundem lobo medio conformis, triente brevior, basi a loborum lateralium marginibus fere vaginatim amplexus. Antherae terminales, introrsae, biloculares, loculis adnatis, in connectivo suboblique lateralibus, connectivo immediatim supra loculos introrsum in glandulam parvam tumente, superne producto in laminam membranaream, substipitatam, anthera multoties majorem, oblongam, obtusam, apice inflexam, marginibus reflexis corohae stamineae processum amplectentem. Pollinia orata, basifixa, retinaculis brevibus, teretiusculis, cum antheris alternantibus geminatim affixa, stigmatis disco incumbentia. Ovarium sessile, ovatum, e carpidiis follicularibus duobus, arcte adpressis compositum, placentis ad suturam ventralem crassiusculis, multiorulatis. Stigma conicum, apice depressiusculum, centro subumbilicatum. Fructus ignotus.

OBSERVAT10. Asclepiadearum ordo naturalis quibus accessionibus, post editam nostram mense Angusto 1838 generum synopsim, sit locupletatus, e sequenti tabula, e manuscripto generum nostrorum supplemento deprompta, adparet.

SUBORDO I. PERIPLOCFAE $R, B r .3431$. Cryptostegia $R$. Br. - 3432. Finlaysnnia Wall. - 3'33. Periploca Linn. - 3'3't. Streptocaulon Wight et Arn. - 3't35. Gymuanthera $R, B r$. - 3'36. Decalepis Wight et Arn. - 343\%. Cornachina Endl. - Brachylepis Wight et Arn, non Hooker et Arn. (infr. $\bar{n}$. 3482.) - 3433. Hemidesmus $17 . B r_{0}-4439$. Lepistoma $B l$. - 3440. Phyllanthera $B l$.

SUBORDO II. SECAMONEAE Endl. 3't't. Secamone $R . B r$. - 3't't2. Toxocarpus Wight et Arn. - 34'43. Goniostemma Wight et Arn.

SUBORDO III. ASCLEPIADEAE VERAE $R$. Br. TRIBUS I. CTNANCHEAE.

§. 1. ASTEPHANEAE. 3444. Hybanthera Endl. Iconogr. t. 63. - 34t5. Astephanus $R$. Br. cfr. Decaisne in Nouv. Annal. sc. nat. IX. 311. - 3't'6. Haemax E. Mey.

S. 2. METASTELMEAE. 3447. Nicroloma R. Br. - 3'48. Parapodium $R$. Br. - 3448 Glossonema Decaisne Op. cit. IX. 335. t. 12. f. d. - 3449. Metastelma $R$. $\bar{B} r . \quad-3450$. Sclubertia Mart. et Zucc. = Pentaphragma Zucc. -3451 . Tweedia Hook. et $\overrightarrow{A r n}$. Bot. Mag. t. 3630. - 3452. Lachnostoma H. B. K. - 3453. Macroscepis H. B. K. - 3454. Pentasachme $\bar{T}$ all. $-\frac{3554}{1}$ Steinheilia Decaisn. Op. cit. 1X. 339. t. 12. $f_{0} e_{0}=$ Asclepias radicans Fork.

S. 3. DITASSEAE. 3455. Eustegia R. Br. - 3456. Sarcostemma R. Br. Endl. Iconogr. t. 64. - 3457. Philibertia H. B. K. - 3458. Doenia R. Br. Decaisn. Op. cit. IX. 336. t. 12. f. f. $-\frac{3458}{T}$ Decanema Decaisn. Op. cit. IX. 338. t. 12. f. g. $=$ Asclepias aphylla Boj. - 3459. Ditassa $R$. Br.

§. 4. CYNOCTONEAE. 3'60. Holostemna R. Br. - 3461. Cynanchum Linn. = Cynanchi sect. 1. R. Br. - 3462. Eudotropis Endl. = Cynanchi sect. 2. R. Br. 3't63. Schizoglossum E. MTey. - 346'\%. Cynoctonum $E$. Mey. = Cyathella Decaisn. Op. cit. IX. 332. t. 12. f. 6. Cynanchi sect. 3., an 4.? R. Br. - 3464 Pycnoneurun Decaisn. Op. cit. IX. 340. t. 12. f. c. $-\frac{3464}{2}$ Morrenia Lindl. in Bot. Reg. 1838. Append. 71. - Cynanchum odoratum Hook. et Arn. - 3465. Vincetoxicum Mönch. = Cynanchum Decaisn. Op. cit. IX. 332. t. 12. f. a. - 3't66. Cordylogyne E. Mey. - 346\%. Solenostemma Hayn. = Argelia Decaisne Op.cit.IX. 331. t. 12. f. .g. - 3468. Glossostephanus E. Mey. - 3469. Metaplexis $R . B r$. = Urostelma Bung. - 3470. Rhysso. lobium E. Mey.

J. 5. CALOTROPIDEAE. 3\$71. Kanahia R. Br. Decaisne in Nouv. Annal. sc. net. IX. 329. t. 11. f. $f_{0}-\frac{3471}{1}$ Oncinema Arnott in Edinb. new philosopl. Journ. 
XVII. 261. - 3'72. Raphistemma Wäll. - 3473. Sentera Reichénb. = Lyonia Ell. -

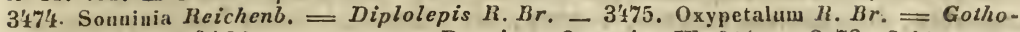
freda Vent. - 3475 Schizostenuna Decaisn. Op. cit. IX, 344. - 3476. Schistogyne Hook. et Arn. - $\frac{3476}{1}$ Calostigma Decaisn. Op. cit. 1X. 343. t. 12. f. h. _, 3477. Arauja Brot. = Physianthus Mlart. et Zucc. - 3178. Calotropis R. Br. Decaisne.Op. cit. IX. 328.

§. 6. EUASCLEPIEAE, 3179. Pentatropis R, Br. Decaisn. Op. cit. IX, 32\%. t. 11. - 3't80. Iphisia Wight. et Arn. - 3't81. Oxystelma $R$. Br. - 3't82. Brachylepis Hook. et Arn. - 3't83 Ensienia Nutt. - Ampelanus Ruf. ='3'4't. Gomphocarpus $R$. Br. Decaisne Op. cit. IX. 32\%. t. 11. f. d. - 3485. Lagarinthus E. Mey. 3'86. Pachycarpus $E$. Mey. - 3487. Xysmalobium R. Br. - 3't88. Acerates Ell. Decaisne Op. cit. IX. 322. t. 11. f. $c_{0}=$ Anantherix Nutt. - Polyotus Nxtlall in Americ. philosoph. Transact. V. 199. - 3'89. Podostigma Ell. - 3'90. Asclepias Linn. 3491. Otaria Funth. - 3492. Pentarhinum E. Mey. - 3493. Aspídoglossuni E. Mrey.

TRIBUS II. GONOLOBEAE. 3'94. Dregea E. MIey. - 3'95. Gonololus L. C. Rich. = Gonolobium Pursh. - 3496. Matelea Aubl. Decaisn. Op. cit. IX, 321 t. 11. f. 6. = Hosta Willd.

TRIBUS IIT. PERGULARIEAE I. SUBTRIBUS I, HOYEAE.

§. 1. SARCOLOBEAE. 3'97. Sarcolobus $R . B r .-3498$. Gymuema R. Br. De. caisne op. cit. IX. t. 11. f. a.

§. 2. TYLOPHOREAE. 3499. Belostemma Wight. - 3500. Tylophora $\boldsymbol{H}$. Br. Decaisne Op. cit. IX. 273. $t$. 10.f. . $_{0}-3501$. Hoya $R$. Br. Decaisne Op. cit. IX. 272. = Schollia Jaeq.f. Sperlingia Vahl. - $\frac{35}{1} \frac{01}{1}$ Centrostenma Decaisne. Op. cit. IX. 271. t. 10. f. c. (Mai, 1838.) = Cyrtoceras Bennett in Horsfield Plant. Jav, rar. 90. t. 21. Hoya multiflora Bl. - $\frac{35}{2} \frac{1}{2}$ Asterostemma Decaisne Op. cit. IX. 271. $t .10$. f. 2. - 3502. Pterostelma Wight. - 3503. Pliysostelma Wight. - 350'. Tenaris E. Mey. - 3504 Fockea Endl. supr. - 3505. Heterostemma Wight et Arn. Decaisne op. cit. IX. 268. t. 10. f. a. - 3506. Cosuastigma Tight́ et Arn. - 3507. Marsieniti R. Br. - 3508. Dischidia $R \cdot \overline{B r}$. = Colyris Vahl. Conchophyllum Bl. - 3509. Lètostemma Bl. - 3510. Stephanotis Thouar. = Isaura Commers. - 3511. Pergularia Linn. Decaisne Op. cit. IX. 2Z6. - 3512. Baxtera lleichenb. = Zlarrisonia Hoak. fi.,

SUBTRIBUS II. STAPELIEAE f. 1. LEPTADENIEAE: 3513. Orthan? thera Wight. - 351'. Leptadenia R. Br. Decaisne Op. cit. IX., 269, t. 10. f. $b$.

5. 2. CEROPEGIEAE. 3515. Fisćheria DC. - 3516. Microstemma $R . B r$. Endl. Iconogr. t. $60 .-3517$. Brachystelna R. Br. - 3518. Sisyranthus E. BLy. - 3519. Ceropegia L. Decaisne Op. cit. IX. 262. t. 9. f. a. b. - 3520: Eriopetalum Wight. w 3521. Bucerosia Wight et Arn. $=$ Desmidorchis Ehrent. Decaisne Op. cit. IX. 265. t. 9. f. c. - 3522. Hutchinia Wight et Arn. - 3523. Caralluiua 11 . Br. Jecaisne Op. cit. IX. 267. $t$. 9. f. d. - 352\%. Stapelia Linn. - 3525. Apteranthes Mick. - 3526. Piaranthus $R, B r .-3527$. Heurnia. $R, B r$.
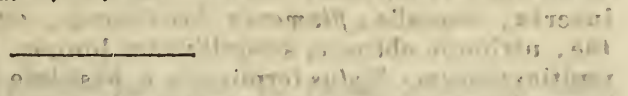

¿2. Cornostylis Juncera Endl, C. perigonio intus glabro, scapis indivisis capitulo vix longioribus, foliis teretiusculis lacvibus.

\section{Colitur in horlo Hügeliano, e seminibus c Novae-Hollandiac} ora austro-occidentali adlatis enata.

Folia in apice caudicis hypogaei disticha, equitantia', sesquipedalia et bipedalia, basi meıbranaceo dilatata, breviter vaginantia, et ibi setis brevibus raris conspersa, snperne teretiuscula, compressiuscula, acuta, juniora versus apicem setis paueis conspersa, adulta glaberrima, laevia, obsolete longitudinaliter striata. Scapus e foliormu breviorum vaginis erumpens, pollicaris, pennae anserinae crassitic, setis candicantibus, patentibus, longruseulis hispidus, apice vestitus bracteis $2-3$, approximatis, late ovatis, semivaginantibus, 6 lineas longis, submembranaceis, viridi nervosis, dorso parce setosis, margine rigidules setoso-ciliatis, intus glaberrimis, in acumen foliacen-viride, planum v. teres, duas lineas longum desinentibus. Flores $3-4$, lutei, in pedicellis vix linea longioribus, setoso hispidis, corymboso-capitati. Perigonii tubns cum ovario connatus, obsolete trigonus, duas et dimidiam lineam longus, setis longius- 
culis, vix ramosis hispidnlus, limbi superi, sexpartiti laciniac erectae, aequales, lineari-lanceolatae, acutae, octo circiter lineas longae, sesquilineam latae, dorso juxta nervum medium apice virescentem parce setosae, intus glaberrimae, nitidulae, basim versus obscurius coloratae. Stamina 6, perigonii laciniis" duarum linearnm altitudine supra eorundem basim inserta, omnia aequalia; filamenta brevissima, antherae lineares, basi subsagittatae, duas et dimidiam lineam longac, erectae, connectivo dursali nerviformi. Ovarium inferum, triloculare. Ovula in placentis glo. bosis, parvis, loculorum angulo centrali insertis plurina. Stylus terminalis, e basi late trigono-conica, lineam longa filiformi triqueter, quinque lineas longus, glaberrimns. Stigma brevissime trilobum, lobis obtusis. Fructum nondum maturavit.

23. Conostylis candicans Endl. C. perigonio intus sublanato, scapis indivisis lanatis folia cano-pubescentia superantibus, spicis geminis secundis.

\section{Novae-Hollandiae austro-occidentalis colonia Swan-River. (Hiigel.)}

Cauliculi triquetri, glaberrimi, uni-bipollicares, decumbentes, apice foliis confertissime distichis onusti. Folia pedalia et sesquipedalia, aversa, basi nonnihil dilatata vaginantia, anguste ensiformia, acuminata, lineam v. sesquilineam lata, striata, pube sericea adpressa candicantia, basi margine interiore ciliata. Scapi e foliorum vaginis exserti, sesquipedales bipedalesque, dense candide lanati, stricti, medio bractea foliacea bipollicari, sericea, margine interiore ciliata instructi, apice folio florali conformi, pollicari muniti, supra folium florale in spicas geminas, secundas, pollicares, patentes divisi. Flores quinque lineas longi, sessiles, fusco-pilosi, pilis ramosis hispidi, singuli basi bractea angustissime lineari, sestertiam lineam metiente stipati. Perigonium campanulatum, sexfidum, tubu ad unius lineae longitudinem cum ovario connato, extus hispidum, intus setoso-sublanatum. Stamina 6 , perigonii laciniis inserta, aequalia; filamenta brevissima, antherac lineares, dorso insertae, utrinque obtusae, sesquilineam longae. Ovarium inferum, triloculare, multiovalatum. Stylus terminalis, e basi late trigono-conica, cava, lincam longa filiformi triqueter, tres lineas longus, glaberrimus. Stigma breviter trilobum, lobis acutis, revolutia.

24. Spadostyles concolor. Endl. Sp. foliis alternis cumeato-oblongis planis obtusis glaberrimis subtus concoloribus, pedicellis dimidio folio brevioribus, bracteolis calycem aequantibus.

\section{Colitur in horto Hügeliano.}

Affinis Spadostyli Sicberi Benth. prima facic foliis et floribus multo minoribus distinguenda. Ramuli pubescenti-hispiduli. Folia alterna, linean v. dimidian lineam sejuncta, oblongo-cuneata, obtusa, quatuor v. quinque lineas longa, unam lineam lata, plana v. marginibus nonnilil involita, utrinque glaberrina, saturste viriclia, subtus concolora, uninervia, marginibus parce hispidula, demum glabrata, apice obtuso brevissime mucronata, basi sensim angustata, et in petiolum brevissimum, vix dimidiam lineam aequantem attenuata. Stipulac petiolum utrinque stipantes, subscariosae, fuscescentes, acuminato-subulatae, vix dimidia 
linea longiores, in unicam intrapetiolarem bidentatam connatae. Flores in suprenorum foliorum axillis solitarii, racemum terminalem brevem, laxun, foliosum constituentes. Pedicelli lineam longi. Calyx laxe campanulatus, sesquilineam longus, parce hispidulus, supra basin bracteolis duabus linearibus, virescentibus, linea paullo brevioribus, suhhispidulis, limbum aequantibus stipatus, ad medium bilabiatus, labii superioris bilobi lobis divergentibus, inferioris tripartiti laciniis lanceolatis, subincurvis. Corollac pallide luteae vexillum rotundatum, alas oblongas paullo superans, carina alas aequans. Stamina libera, glabra. Ovarium glaberrimum. Stylus basi compressus, subdilatatus, basi postice sericeo villosus, caeterum glaberrimus, apice nonnihil incurvus.

\%5. Spadostyles romanosa Endl. Sp. foliis alternis oblongo-obovatis planis obtusis hispidulis, pedicellis brevissimis, bracteolis calycem aequantibus.

\section{Colitur in horto Hügeliano.}

Rami, ramulique breves, confertim alterni, strictiusculi, hispidi. Folic approximatim alterna, erecto-patentia, oblongo-obovata, tres et dimidiam lineam longa, unam lineam lata, obtusa v. retusa, plana, margine interdum subinvoluta, ntrinque saturate viridia et hispidula. Stipulae scariosac, in unicam interpetiolarem, acuminato-bidentatam connatac. Pedicelli in suprenorum foliorum axillis solitarii, dimidiam lineam longi. Calyx campanulatus, hispidulus, duas lineas longus, labii superioris lobis divergentibus, inferioris tripartiti laciniis patentibus, omnihus acutis, subulatis. Corollae luteo-subaurantiaceae vcxillum late rotundatum, basi macula pallide purpurea, alas oblongas, obtusas vix superans, carina alas aequans. Ovarium glaberrimum, compressum. Stylus compresso-dilatatus, basi fasciculo pilorum caudidorum stipatus, caetcrum glaberrimus.

26. IBosสinea Ovalifolia Eadl. B. ramis leretibus foliosis foliisque ovali-ellipticis mucronatis subtus villosis, stipulis petiolum superantibus, pedicellis folio brevioribus.

\section{Colitur in horto Hügeliano.}

Rami teretes, tenelli, villosi. Folia alterna, duarum linearum vesquiliweac intervallis remota, ovali-elliptica, quatuor ad sex lineas Inga, duas et dinidiam, v. tres lineas lata, subcoriacea, supra glabra, subtus imprimis ad nervum villosa, utrinque transversim venosa, reticulata, apice in mucronem rigidnlum, quadrantem lineae superantem desinentia, marginibus subrecurva, basi subemarginata, petiolo unius lineae longitudinem aequanti, filiformi inserta. Stipulac petiolorum basim utrinque stipantes acuminato - subulatae, subsetaceae, duas lineas longae, fuscae. P'edicclli in axillis foliorum solitarii, tres v. quatuor lineas longi, basi bractcola squamaeformi obtusa, supra basin bracleolis iuabus ovato-lanceolatis, et ad unedium altero bracteolarum angustissine linearinm, villosissimarum pari stipati, Calycis (in alabastro acuminati) villosi, a latere compressiusculi, et dorso acute convexi, ultra medium bilabiati labiun superius bilobum, lobis longitudine seste:tiam lineam aequantibus, acmuinatis, divaricato-patentibus, inferioris tripartiti laciniis anguste lanceolatis, 
acuminatis, labium superius aequantibus, erectiusculis, Corollae vexillum ungue lineam longo, dimidiam lineam lato, lamina patente, quinque lineas lata, tres lineas longa, vix emarginata, intus aurea, margine et dorso praeter basim sanguineam fusco purpurascens. Alac oblongae, obtusissimac, tres et dimidiam lineam longae, unam lineam cum quadrante latae, basi excisae, hinc in unguem filiformem angustatae, inferne rubentes, superne cum flavore suffuso sordide purpurascentes. Carinac petala longe stipitata, alis paullo longiora. Stamina monadelpha, glabra, antherae uniformes, luteac. Ovarium sessile, compressum, dense sericen-villosun. Stylus filiformis, brevis, praeter basim hinc villosan glaber. Stigma miuutum.

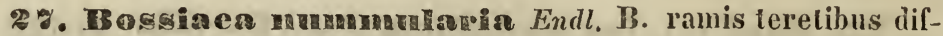
fusis foliosis, foliis ovato-suborbiculatis mucromulatis glabris, stipulis petiolum aequantibus, pedicellis folia subsuperantibus, calycibus leguminibusque glabris.

\section{Colitur in horto Hüyeliano.}

Ramuli filiformes, teretes, foliosi, parce puberuli. Folia ovato-suborbiculata, tres lineas longa, duas et dimidian lineam lata, mucromulata, tenera, superne glaberrima, juniora subtus sub lente pilis raris adpressis conspersa, uninervia, obsolete venosa, brevissine mucronulata, petiolo brevissimo, vix dimidiam lineam aequante, apice incrassato. Slipulac subulatae, fuscae, petiolum aequantes. Pedicelli filiformes, tres lineas cum quadrante metientes, floriferi deflexi, fructiferi strictiusculi, sub lente pubescentes, basi bractea squamaeformi, medio bracteolis, dimidiam lineam longis amplexi. Calyx (in alabastro obtusiusculus) duas lineas longus, glaber v. sub lente parce puberulus, dorso convexus, lahii superioris lobis latis, divaricato-patentibus, inferioris laciniis lanceolatis, erectiusculis. Vexilli lamina dnas et dimidiam lineam longa, quatuor lineas lata, emarginata, intus lutea, extus rubescens. Alae obtusac, carina flavescente paullo breviores. Legumen compressum, breviter stipitatum, glaberrimum, junius ad suturas subvillosum.

ANTICHARIS Endlicher Gen. plant. n. 3944. Calyx ebracteolatus, quinquepartitus, subaequalis. Corolla hypogyna, bilabiato-quinquefida, lobis subaequalibus, obtusis. Stamina 2, corollae tubo antice inserta, inclusa; filamentis brevissimis, antherarum loculis divaricatis, demum confluentibus, inaeyualibus. Ovarium biloculare, placentis dissepimento utrinque insertis, multiovulatis. Stylus filiformis, simplex; stigma obtusum. Capsula ovato-pyramidata, bilocularis, loculicide bivalvis, valvis demum septicide bifidis, placentis coadunatis, tandem liberis. Semina plurima, ovata, costata. - Herba arabica, subsimplex, glandulosopubescens; foliis alternis, ellipticis, integerrinis, in petiolum angustatis, pedunculis axillaribus, solitariis, unifloris, infra medium minute bibracteolatis. 
28. Antichanis arabiea Endl. Iconogr. Gen. pl. t. 93.

'Capraria arabica Steudel et Hochstetter msc.

In Arabiae felicis montibus Sidr et Kasr, in saxis collium Ged-Lac vicinorum legit S. Fischer. (Herb. Arab. Nr. 18.) - In rupibus prope pagum Ferihe Arabiae felicis legit W. Schimper. (Herb. Union itiner. 1837. n. 848.)

Herba annua, pedalis v. sesquipedalis, simplex v. inferne ramis paucis, ut plurimum brevibus aucta, erecta, stricta, omnibus partibus pube glandulosa, subviscida conspersa. Radix exilis, flexuosa, simplex, brevissima, vix fibrillosa. Caulis teres, fili emporetici tenuioris crassitic, sursum sensim attenuatus, basi sordide purpureus, superne glauco-viridis, pilis brevibus glanduloso-viscidis, patentibus consitus, strictus $v$. inter folia flexuosus. Folia quatuor linearun intervallis alternantia, elliptica, obtusiuscula, novem circiter lineas longa, duas et dimidiam lineam lata, integerrima, in petiolum bilinearem angustata, subtus uninervia, utrinque pube glandalosa brevi viscidula, glaucescentia. Pedunculi in foliorum, saepissime ramuli abortivi folia prima minuta evolventium, axillis solitarii, uniflori, filiformes, tres lineas longi, erecto-patentes, cum calycibus paullo quam reliquae plantae partes confertius glanduloso-pubescentes, infra medium bracteolis duabus linearibus oppositis, vix dimidia linea longioribns muniti. Flores duas lineas longi. Calycis quinquepartiti laciniae lineari-lancelatae, acutae, lineam longae, duae anticae paullo breviores, omnes erectae, confertim glanduloso-pubescentes. Corolla hypogyna, calycis dupla longitudine, infundibuliformi tubulosa, sursum sensim ampliata, limbo breviter quinquefido, subbilabiato, lobis rotundatis, obtusissimis, duobus posticis duobusque lateralibus aequilongis, intermedio antico reliquis circiter triente longiore, omnibus erectis, venosis. Stamina 2 , medio corollae tubo, inter limbi lobum anticum et laterales inserta, brevissina, nec faucem attingentia, omnino inclusa. Filamenta hasi nonnihil dilatata. Antherarum loculi divaricati, mox confluentes, filamenti apici in ferri equini formam circumplexi, cruribus obtusis, altero plus triente longiore. Ovarium ovatum, glaberrimum, tertian tubi corollini partem longitudine vix aequans, biloculare. Ovula in placentis carnosis, linea dorsali angusta medio dissepinento utrinque insertis plurima, horizontalia. Stylus terminalis, filiformis, ovario triplo longior, faucem corollae aequans. Stigma terminale, obtusum, vix emarginatum. Capsula membranacea, ovato-conica, acuta, tres lineas cum dimidia longa, bisulca, calyce persistente stipata, stylo superata, substraminea, obsolete venosa, parce glanduloso puberula, bilocularis, loculicide ab apice ad basin bivalvis, valvis medio septiferis, mox fere ad mediam sui longitudinem septicide bifidis. Placentae in unicam ovatam, ab altera valvularum tardius solutam, mox penitus liberam, scrobiculatam coalitae. Semina plurima, ovata, sulghlobosa, spadicea, loncritndinaliter costis acutiusculis elevatis, subduodenis exarata, transversim striato-subfasciata, costis apice in apiculnm brevissinum convergentibus, basi sensim circa discum, medio micropyle centrali minutissima apertum, et umbilico excentrico impresso notatum deliquescentibns. Embryo in axi albuminis carnosi orthotropus, magnus, subclavatus, seminis longitudine; cotyledonibus crassinscule plano-convexis, obtusis, radicula basi nonnilil incrassata, micropylem attingente, umbilico parallele contigua.

\$2. Thelymaitre Gexwosa Fndl. Th. perigonio patulo flavo, cuculli laciniis lateralibus erectis obtusis integerrimis nudis, intermedio truncato crenulato, foliis lincaribus canaliculatis, caule flexuoso. 
Crescit in Novac-Hollandiae austro-occidentalis colonia KingGeorges-Sound. (Hügel.)

Bulbi radicales gemini, ovati, putaminis Cerasi mole, sordide fusci, alter minor, exsuccus, alter tumidus, panllo altius insertus, apice squa.mis laceris, e caulis basi dependentibus quasi calyptratim tectns. Caulis sex-octopollicaris, tenellus, glaberrinus, basi vagina semipollicari membranacea, truncata inclusus, praeter folium radicale et floralia duobus aliis aequis intervallis dissitis instructus, ad folia geniculato-flexus. Folia anguste linearia, acuta, canaliculata, basi longe vaginantia, vagina caulem arctissime ambeunte, glaberrima, radicale e caulis vagina exsertum, duos et dimidium pollicem longum, caulina pollicaria, obtusiora, floralic laxiora, latiora, obtusa, tres lineas Ionga. Flores in speciminibus obscrvatis constanter nonnisi duo, alter ex axilla folii floralis breviter pedunculatus, alter in pedunculo longiore, caulem continuante, prope apicem folio florali minore instructo. Perigonium patens, foliola exteriora ovata, tres lineas longa, duas lineas lata, interiora paullo minora, obtusiora. Labcllum conforme, concolor. Columna membranacea, duas lineas longa, marginibus conniventibus, lutea. Cuculli tripartiti, sordide rubentis lobi laterales dimidiam lineam longi, compressi, erecti, obtusi, glaberrimi, intermedius brevior, truncatus, obsolete crenulatus. Anthera lobi intermedii basi inserta, obtusa, lineam longa. Ovarium tres lineas longum, cylindrico-subclavatum, sexstriatum, glaberrimum.

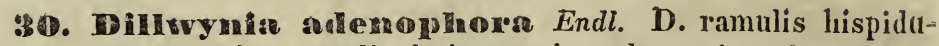
lis, foliis teretiusculis canaliculatis rectis suberectis tuberculosopunctatis hispidulis muticis v. vix mucronatis, floribus axillaribus sessilibus approximatis, pedicellis calycis tubo multo brevioribus, dentibus calycinis glandula capitata terminatis.

\section{Colitur in horto Hügeliano.}

Species perpulchra, D. floribundae proxima, dentibus calycinis glandula capitata terminatis facillime distinguenda. Ramuli stricti, rubri, pilis candicantibus, patentiusculis hispiduli. Folia confertim sparsa, recta nec ullo modo torta, erectiuscula, semiteretia, dorso convexa, facie canaliculata, quinque v. sex lineas Ionga, mutica, obtusiuscula v. juniora in mucronem brevissinum, glandula capitata terminatum desinentia, tuberculis aspera, pilis candicantibus, patentiusculis, mollibus hispidula. Flores in axillis foliorum solitarii v. gemini, approximati, racemum brevem apice comosum formantes. Pedicelli brevissimi, vix dimidiam lineam Ingi, bractea bracteolisque geminis, dimidio calycis tubo longioribus, squamaceis, hispidulis, caducissinis stipati. Calyx campanulatus, tres et dimidiam lineam longus, pilis adpressis hispidulus, labio superiore lineam longo, sesquilineam latitudine paullo superante, brevissime bilobo, lobis divergentibus acutiusculis, glandula capitata terminatis, margine ciliatis, inferioris paullo brevioris, tripartiti laciniis triangularibus, acutis, omnibus glandula capitata pedicellata superatis. Corollae petala medio calycis tubo inserta, decidua. Vexillum ungue duas lineas longo, $3 / 4$ lin. lato, lamina patente, sex lineas lata, emarginata, extus aurantiaceo rubra, intus anrantiacea, basi sanguinon picta. Alae sanguineae, oblongae, obtusae, tres lineas longae. Carina recta, obtusiuscula, duas lineas longa. Stamina glaberrima, libera : decidua; filamentis basi latioribus. Ovarium brevissime stipitatum, sericeum, biovulatum. Stylus e basi compressa angustatus, apice uncinatus; stigmatc terminali capitato. 
Nr. 4.

\section{NOVARUII STIRPIUII}

\section{DECADES}

\section{Editac a Museo Caesareo palatino Vindobonengi.}

Nr. 4. Vindobonae, typis Sollingerianis. 30. Maji 1839.

MANGLESIA Endlicher Gen. plant. Suppl. n. $\frac{2142}{1}$. Perigonium tetraphyllum, foliolis subspathulatis, demum revolutis. Antherae 4, apicibus concavis foliolorum perigonii immersae. Glandula hypogyna semiannularis. Ovarium stipitatum, uniloculare, biovulatum. Stylus supra basim declinatam incrassatus; stigma conicum. Folliculus coriaceus, abortu monospermus. Semen apterum. - Frutices Novae-Hollandiae; foliis pinnatifidis v. lobatis, glandulis cutaneis amphigenis v. hypogenis, racemis terminalibus lateralibusque corymbosis, pedicellis filiformibus, geminatis, paribus unibracteatis, basifloris.

Genus, ad quod praeter species novas infra describendas, et Manglesiam cuneatam Endl. (quae Grevillea Manglesii Hortul.), verisimiliter omnes Grevilleae sectionis Conogyne R. Brown Sup. plem. 21, a Cunninghamio ad Anadeniam relatae, erunt referendae, a Grevilleis legitimis perigonio regulari, stylo et stigmate diversum, ab Anadenia glandulae hypogynae praesentia, et stylo supra basim filiformem incrassato maximopere differt.

Genus dico honoribus Jacobi Mangles, Navarchi regii bricannici, et Roberti Mangles Eq. in Sunning-Hill, quorum cura plurimae stirpes e Novae-Hollandiae austro-occidentalibus in Angliam advectae, jam hortos nostros exornant.

31. Mangiesia tridentiferea Endl. M. glaberrima, foliis trifurcatis, laciniis subulatis dorso convexo et facie plana bisulcatis mucronatis pungentibus indivisis bi-trifurcatisque, corymbis axillaribus simplicibus folio brevioribus.

Habitat in Novae-Hollandiae austro-occidentalis interioribus, inter Swan-River et King-Georges Sound. (Rö̈.)

Fruticis ramos video pedales, teretes, glaberrimos, lacves, pennac scriptoriae crassitie, ramulis aliquot multo tenerioribus, patentiusculis, subfastigiatis auctos. Folia alterna, basi filiformi-trigona, tre v. quatuor lineas longa, apice trifurca, laciniis dimidinm v. unum pollicem longis, 
suluulatis, patentissinis, rigidis, glaberrimis, dorso convexo ct facie plana bisulcatis, in mucronem sphacelatum, pungentem desinentibus, indivisis, v. uno alterove breviter bi- rariusve trifurco. Racemi corymbosi, axillares, solitarii, folio breviores. Flores minuti, sesquilineam longi, glaberrimi, ex sicco flavescentes. Pedicelli filiformes, geminati, paribus bractea squamaeformi, minuta, caducissima sufiultis, fastigiati, tres ad sex lineas longi, flaccidiusculi. Perigonii tetraphylli foliola sesquilineam longa, hasi angustata, subspathulata, infra apicem orbicularem concavum, staminiferum constricta, aestivatione valvata, pedunculum obconicum, capitulo globoso terminatum referentia, sub anthesi prinum inferne inter se distincta, apicibus diutius cohaerentia, mox revoluta, cito decidua. Ovarium compressiuscule subglobosum, pedicello filiformi, basi glandula unilaterali truncata stipato, dimidiam lineam longo excentrice impositum, uniloculare, biovulatum. Stylus juxta ovarii latus quo illud pedicello est affixum excentrice terminalis, supra basim filiformem, incurratam inflatus, sursum attenuatus; stigmate late conico, obtuso terminatus.

3:. Manglesia vestita Endl. M. ramis tomentosis, foliis coriaceis cuneatis apice trifidis, lobis indivisis mucronatis pungentibus, utrinque imprimis subtus hirsutis, corymbis axillaribus simplicibus folio longioribus.

\section{Habitat in Novae-Hollandiae austro-occidentalis colonia} King-Georges-Sound. (Hügel.)

Rami teretes, ramulique breves, dense cinereo-tomentosi. Folia alterna, coriacea, cuneata, septem circiter lineas longa, quatuor lineas maxime lata, basi vix lineam metientia, apice trifida, lobis acutis, tres lineas longis, in mucronem pungentem, dimidia linea longiorem desinentibus, marginibus revoluta, utrinque, imprimis subtus hirsuta, adultiora supra subglabra, pilis centro affixis. Corymbi axillares, densi, breve pedunculati, novem circiter lineas longi, rhachi tomentosa. Flores duas lineas longi, omnino ut in praecedente specie. Ovarii pedicellus basi glardula unilaterali, truncata stipatus, tres quartas lineae partes metiens. Stylus supra basim filiformem inerassato-inflatus, sursum attennatus, stipiti ovarii aequilongus. Stigma conicum, acutum, longitudine sua dimidium stylum aequans.

33. Dolonaen Inamilis Endl. Atakt. $t$. 31. D. foliis impari-pinnatis tri-quadrijugis, foliolis oppositis cuneatis apice truncato inciso - dentatis petiolisque alatis glabriusculis, floribus terminalibus umbellatis, stylis elongatis, capsulis apteris echinatis.

Crescit in Novae-Hollandiae ora australi, loco dicto MemoryCove. (Ferd. Bauer.)

Fruticulus humilis, cujus summitates video palmares, viscidnlas, ramosas, ramis alternis, angulatis, strictiusculis, subfastigiatis. Folia alterna, impari-pinnata, quadri-quinquejuga, octo ad decem lineas longa, quinque lineas lata, circumscriptione obovata, laevia, glabriuscula, supra obscure viridia, subtus pallidiora. Folioia opposita, duas v. tres lineas dissita, cuneata, duas et dimidiam lineam longa, sesquilineam v. duas 
lineas lata, inferiora multo minora, superiora sensim majora, omnia apice truncata, inciso-dentata. Peliolus alatus, unam lineam latus, ala ad foliolorum insertionem interrupta. Flores in diversis stirpibus masculi a femineis sejuncti, in ramulis terminales, umbellati, umbellis subquadrifloris, basi bractea foliacea, foliis rameis omnino conformi, sed paullo minore suffultis. Floribus masculis : Caly $x$ quadripartitus, laciniis ovatis, acuminatis, parum carinatis, extus dorso marginibusque pilis rigidulis longiusculis raris conspersis. Stamina octo, primum erecta, demum cx trorsum nutantia. Filamenta filiformia. Antherae luteae, laciniis calycinis duplo fere longiores, mutua pressione tetragonae, utrinque obtusae, bi. loculares, loculis oppositis, utroque latere juxta totam longitudinem dehiscentibus, acumine filiformi, e connectivo inter loculos transeunte terminatac. Florcs feminei grani cannabini mole, sordide purpurascentes. Calycis quadripartiti laciniae ovatae, acuminatae, planae, erectac, solo apice patentes. Ovarium calyce dimidio brevius, globosum, laete viride, papilloso-muricatum, quadriloculare, loculis biovulatis, ovulo superiore adscendente, inferiore pendulo. Siylus teretinsculus, longe exsertus, calyce quadruplo longior, purpureus, apice breviter quadridentatus, dentibus patentiusculis, intus stigmatosis. Capsula Pisi majoris mole, globosa, muricato - echinata, quadrilocularis, quadrivalvis, axi centrali tctraptero. Semina in loculis bina, axeos faciebus affixa, superius adscendens, inferius pendulum, compressa, orbiculata, crista carnosa cincta.

IRIBONANTHES Endlicher Gen. plant. Süplem. $\boldsymbol{n} . \frac{1259}{1}$. Perigonium arachnoideo-lanalum, tubo basi cum ovario connato, supra ovarium producto, limbi sexpartiti laciniis patentibus. Corona faucis petaloidea, hexaphylla, foliolis perigonii laciniis oppositis, exsertis, cuneatis, trifidis, lobo medio basi antherifero. Stamina 6 ; filamenta nulla, antherae foliorum coronae lobo dorso insertae, inclusae, lineares, sagiltatae. Ovarium inferum, triloculare. Ovula in placentis e loculosum angulo centrali prominentibus plurima, amphitropa. Stylus e basi dilatato-conica cylindricus; stigna fusiforme, brevissime trilobum. Fructus ..... Herba Novae-Hollandiae austro-occidentalis; tuberibus radicalibus ovatis, subfasciculatis, caule simplici, apice lanato, oligophyllo, foliis aversis, inferne planis, apice teretibus, basi vaginantibus, lo- $^{-}$ ribus terminalibus, capitato-congestis, bracteatis.

\section{Trobonathes augtrolis Endl.}

\section{Habitat in Novae-Hollandiae austro-occidentalis colonia} King-Georges-Sound. (Hügel.)

Herba palmaris v. pedalis, simplicissima. Tubera radicalia gemina, orchidea, ovata, Pisi majoris mole, unum exsuccum, corrugatum, in caudicem hypogaeum brevissimum immediatim transiens, alterum plerumqne minus, breviter stipitatum, turgidnm, laeve. Caudcx hypogaeus vix dinidium pollicem aequans, vaginis membranaceis laxinsculis, laceratis tunicatus, ima basi fibras unipollicares, flaccidiusculas, flexuosas, compresse filiformes, quandoque medio nonnihil incrassatas, parce fibrilliferas confertim exirens. Caulis pedalis, simplicissimus, strictus, diphyllus, folio altero subradicali, altero medio cauli inserto, striatus, glaber, nonnisi infra terminale florum capitulum dense arachnoideo-lanatıs, basi vagina membranacea, laxa, nitidula, in apicem subfoliacenm, planum, obtusum, nunc brevissimum, quandoque dimidium fere pollicem 
a equantem desinente inclusus. Folia arersa, inferne plana, apice teretia, acutiuscula, giabra, laeria, obsolete striata, alterum subradicale, duos et limidium pollicem longum, latitudine maxima vix linea latius, apice teres, basi laxe vaginatim caulem amplectens, ibique submembranaceo marginatum, in vaginam integram usque ad caulis basim pertingentem decurrens, alterum medio cauli, rarius in speciminibus nanis nondum rite evolutis paulo infra florum capitulum insertum, vix pollice longins, teres, acutum, baseos dilatatae marginibus membranaceis subundulatis, caulem laxe amplectentibus, inferne in raginam dnas et dimidiam lineam vix superantem connatis. Flores in apice caulis 2-5, dense capitato-congesti, singuli basi bractẹa squamacea, fusccscente, ovata, obtusa, quatuor lineas longa, duas lineas lata, glabra, solum marginibns arachnoideo-lanata, intus trinerri, perigonio adpressa stipati. Perigonium lana arachnoidea densa, alba, hinc inde derasa vestitum, tubus tres lineas longus, cylindricus, ad tertiam longitudinis suae partem cum orario connatus, parte supra ovarium producta intus glaberrima, laevi, nitida, limbi sexpartiti laciniae oblongae, quatuor lineas longae, duas lineas latae, acutae, patentissimae, utrinque lanatae, lana minus quam in tubo densa, in pagina superiore saepius hinc inde derasa. Corona hexaphylla, summae fauci inserta, foliola petaloidea, laminae perigonii tubum intus vestienti continua, limbi laciniis opposita, exserta, erecta, glaberrima, ex sicco fusco-rubra, cuneata, duas lineas longa, latitudine maxima lineam unam metientia, paullo ultra medium trifida, laciniis lateralibus linearibus, obtusis, integerrimis $\mathbf{v}$. interdum breviter bidentatis, dente interiore nano, lacinia intermedia nunc lateralibus latiore, biloba, lobis apice inciso bidentatis, nunc abbreviata nana, inaequaliter bi-trifida. Antherac foliolornm coronae lobo intermedio supra eorundem basim medio dorso insertae, lobis lateralibus saepe conniventibus relatae, introrsae, biloculares, lineares, unam lineam longae, apice brevissine apiculatae, hasi sagittato-bilobae, loculis appositis, introrsum longitudinaliter dehiscentibus. Ovarium inferum, lineam longum, triloculare. Ovula in placentis ovatis, carnosis, e medio angulo centrali prominentibus plurima, amphitropa. Stylus sesquilinearis, e basi conico-dilatata cylindricus, glaberrimus. Stigma dimidia linea paullo brevius, fusiforme, glabrum, apice brevissime tridentatum.

35. Crumenaria erecta Reissck. C. suffuticosa perennis, caulibus erectis strictis, foliis minimis oblongo-lanceolatis amplexicauli-sessilibus, stylo trifido.

\section{Habitat in pascuis ad Rio de S. Francesco Brasiliae (Pohl.)}

Rhizoma pollicis fere crassitiem attingens, lignosum, supra ramosum, emortuis caulium fasciculis nodosum, nodis nonnonquam in massam spongiosam excrescentibus, siccum saporis parum acris. Caules in fasciculos densos collecti, pedales, fructiferi sesquipedales, fasciculis in rhizomate simplici singulis, in ramoso pluribns. Caules porro annui, interduın biennes, erecti; exteriores subinclinati, graciles, stricti, herbacei, glabcrimi, in sicco striati. Ramuli statim a basi sub angulo valde acuto prodeuntes, erecti, simplices, caule breviores ipsique conformes. Folia stipuliformia, respectu plantae minima, saepe lineam tantum longa, nune patentia, nunc ramo adscendenti adpressa, e basi sessili fere amplexicauli oblonga, rel oblongo-lanceolata, integra aut hic inde minute repando denticulata, crassiuscula, glabra, interdum margine inferiori setulis paucis stipularum ciliis similibus inspersa, obtusa rel cuspidata, subtus verruculosa, nervo parum prominulo. Stipulae dimidiae plerum- 
que folii longitudinis, lineares, obtusae, setis albicantibus ciliatae, apice barbatae. Pedunculi in apicibus ramulormm fasciculati, vel fasciculis spar:sis in caule sessilibns, supremi conferti, fere capitati, omnes filiformes, teneri, patentes vel erecti, pilis parvis muniti, flore parum longiores. Bracteae propriae nullae, sed stipulae ad apicem cauliun usque fasciculıs comitantes, ibique ad basim pedunculormm bracteae communes evadunt, licet et in pedunculis nonnunquam bracteola valde minuta tennis, acu ta conspicienda. Calyx lineae Iongitudinem aequans vel superans, campanıalatus, sub anthesi ampliatus, serius in medio contractus, deciduus, basi pilis rigidis septatis cinctus, supra glaber, in lacinias triangulari-ovata s, acutas vel obtusiusculas, dimidio ipsius breviores divisus. Laciniae intis nervo e basi calycis veniente, versus apicem incrassato, supra nervulis aliis duobus lateralibus prius extus flexis, dein conniventibus comitatio, percursae. Petala cucculiformi-convoluta, tenera, lacinias calycinas longitudine plurimum excedentia, in unguem brevem angustata. Stamiza petalis inclusa. Filamenta basi subinflexa, teretia. Antherae globosae, vel ovato-globosae antice dehiscentes. Ovarium calyci adnatum, obovatum subconvexum, perianthio decidno margine elevaio supra cinctum. Stylus trigonus, inferius crassior, superius in apicem trifidum productus. Strigmata tria, divergentia, papillosa.

OBSERTATIO. Species primo acispectu a $C$. decumbente Mart. habitu va.lde diversa, quod praecipue caulibus fasciculatis, exiguitati foliorum et inflorescentiae tribuendun. Adcuratiori observatione autem in structura stipularum et florum magnam in. venimus analogiam, et certe etiam fructus haud diversus. Verosimile autem in vastis Brasiliae regionibus plures adhuc formas intermedias latere,

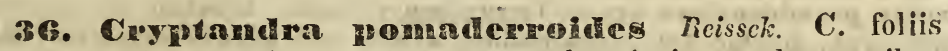
obovatis ovalibusque integerrimis supra brevissime pubescentibus sublus tomentosis, floribus terminalibus capitatis, calyce tubuloso.

\section{In Novae-Hollandiae austro-occidentalis interioribus legic} cl. Rö̈.

Fruticulus humilis (?), ramis castaneis, albido-flávescenti punctatis, annotinis tomento densissimo ferrugineo, tandem evanescente velutinis. Folia breviter petiolata, plerumque obovata, obtusissima vel leviter emarginata, integerrima, crassiuscula, juniora supra pube perbrevi, liberis oculis hund conspicua tecta, adultiora tomento anisso paginam tantum verrucis minutis obsitam, scabriusculam ostendunt. Pagina inferior cum petiolo dense tomentoso villosa, et praecipue in costis venisque prominulis pilis fiongioribus, sericeis, purpureo-fuscis inspersa, tomento marginem excedente folia quasi dense ciliata, pilis serius evanescentibus simpliciter albo tomentosa. Stipulae aliae ovato-lanceolatae, cuspillatae, aliae fere lanceolato-subulatae, spadiceae, margine subrevolutae, carinatae, dorso albo villulosae. Capitula foliis pluribus congestis plerumque cincta, densiflora, hemisphaerica. Bracteae subrotundo-vel lato-ovatae, cuspidatae, scariosae, stipulis concolores, carina villosae, calyce breviores. Calyx tubo eylindrico, lineae longitudinem fere aequante, supra in lacinias triangulari ovatas, acutas, sub anthesi erectoppatentes, intus carina subflexuosa, apice incrassata percursas divisus. Tubus extus pube simplici villosissimus, villis in laciniarum apice longioribus, intus glaber, albo flavescens. Petala minuta, subgaleata, breviter ungniculata, lirunea. Stamina petalis inclusa; filamentis brevissimis, teretibus, basi incrassatis, antherac ovatae, intus dehiscantes. Ovarium fundo calycis omnino fere adnatnm, superne glabrescens. Stylus simplex, versms apicem vix attenuatus, calycis faucem attingens, stigmate tridentato. 
OBSERVATIO. Species cum Cryptandra tubulosa Fenzl ad sectionem alteram hujus generis : calyce tubulosa pertiuet, et praeterea ovario ounino fere adnatol insignis, ex quo concludere licet etiam in fructu differentian adesse, quo autem non viso, speciem hanc generice separare haud audeo.

3\%. Pomalerrois Motre Reissek. D. foliis ovato- et elliptico-lanceolatis integris supra hirtis subtus cum ramulis petiolisque densissime- albido vel subferrugineo-tomentosis, tomento scabriusculo, floribus corymboso-cymosis, cymae ramulis angulato striatis, striis denudatis, calycibus villosis, petalorum lamina oblonga.

\section{Habital in Nova-Hollandia orientali extratropica. (Hïgel.)}

Frutex ramulis plus minusve hirsuto-tomentosis, tomento e pilis rigidiusculis, ferrugincis, tandem albescentibus formato. Folia petiolo crasso, valide tomentoso insidentia, e basi ovata vel rotundata lanceolata, suprema tantum elliptico-lanceolata, omnia integra, vix hinc inde minutissime repando-subdenticulata, acuta. Pagina superior scabra, verrucis minimis conspersa, et setulis albicantibus simplicibus, raro ramosis hirta, inferior dense albo-tomentosa, pilis longioribus ferrugineis praesertion in costis venisque prominulis, et versus marginem immixtis. Stipulae ovatae, scariosae, carina albo-tomentosae. Cyma terminalis, corymboso-paniculata, subdivaricata, ramulis di- aut trichotomis, striato angulatis, striis non penitus quidem nudis, sed tomento tantum tenui, albo involutis, costis prominentibus dense ferrugineo-villosis. Bracteac involucrantes concavae, ovatae vel subrotundae, acutae vel obtuse scariosae, flores et cymae ramulos amplectentes, bruneae, dorso linea villoso-tomentosa notatae, versus marginem subglabrae et pallidiores, in ipso margine plerumque dense ciliatae, intus glaberrimae. Flores in qualibet bractea plerumque tres, breviter pedunculati. Calyx urceolatus, externe longe albo-villosus. Petalorum lamina oblonga, subconcava. Ovarium apice albotomentosum, stylum inferne tomento reconditum, stigmatibus tribus exserens.

OBSERVATIO. Species vix cum aliis confundenda, $\boldsymbol{P}$. lanigerae Sims. et P. ferrugineae Sieb. quidem affinis; differt tanen a priori: foliis plerumque ovato lanceolatis supra verruccosis hirtisque, nec pube simplice densa et mollissima conspersis; subtus pilis longioribus tonento albo immixtis, quum in $\boldsymbol{P}$. lanigera tomentum ferrugineun simplex siue inspersis pilis longiorihus observetur; praeterea in nostra ramulorum to. mentum longius et densius, cymaeque laxiores sunt. P. ferruginea Sieb. ab hirta differt: folis supra glabris, subtus simpliciter tomentosis et floribus fere paniculatis. A. $\boldsymbol{P}$. apetala Labill. et $P$. aspera Sieb., quibus nostra respectu aliquo similis, praesentia petalorum, forma tomentoque foliorum, alisque notis facillime dignoscitur.

38. Tizyphus Garolneri Reissek. Z. foliis ovatis dentatis utrinque glaberrimis nitidis, trinerviis, aculeis solitariis rectis petiolo subaequalibus, floribus corymbosis glabris sub anthesi petiolo vix longioribus bracteolis minutis suffultis, stigmatibus subcapitatis.

Habitat in Brasilia. (Gardner.)

Arborea (?) Ramuli diffusi, subflexuosi, teretes, glabri, parum rerruculosi, epidermide cinerea tecti, qua evanida bruneo-ferruginei 
adparent, juniores albido-punctati, glabri, vel subinde setulis valde minutis, ferrugineis inspersi. Folia petiolo brevi, plerumque setulis bruneis unito, sparsa, alterna, inferiora ovalia obtusa, vel leviter emarginata, superiora ovato-acuta, aut acuminata, margine subaequaliter dentata, denticulis acutis vel obtusis, omnia glaberrima, nitida, infra trinervia, nervi validiores costaeformes, nonnunquam pilis inspersi. A basi et a lateribus nervorum nervuli alii versus marginem excurrunt, et cum venis multis prominulis rete tenerum in pagina inferiori efformant. Aculei hinc inde folia comitantes, recti, subteretes, crassitie et longitudine fere petioli. Flores in corymbos parvos, axillares collecti. Pedunculus communis petiolo brevior, et simili modo setulis ferrugineis patentibus conspersus. Rami corymborum divaricati, pedunculos simplices breves, fasciculatos, versu's apicen incrassatos, pilis valde exiguis albicantibus diaphanis hinc inde consitos gerentes, bracteolis minimis brunescentibus ovatis, obtusis, pilosiusculis mox deciduis suffuitos. Calyx tubo obconico, expanso, laciniis patentissimis, triangulari-acutis, interne linea elevata, subflexuosa percursis. Petala convoluto-conchata, in unguem lamina longiorem attenuata, reflexa. Staminum filamenta margini disci inserta, recurva. Discus pentagonus, crassus, carnosus, in medio ad receptum ovarii perforatus. Ovarium disco immersum, nec adnatum, biloculare. Styli duo infra crassiores, approximati, supra attenuati, divergentes, quilibet stigmate subcapitato instructns.

OBSERVATIO. Species descripta $Z$. Loto et $Z$. Spina Christi affinis, differt autem a priore: aculeis solitariis rectis, ramulis glabriusculis, corymbis densioribus, pedunculis sub anthesi flore vix longioribus; praeterea folia in $Z$. Gardneri magis denrata, quum in $Z$. Loto tantum subdenticulato-crenata observantur. $Z$. Spina Christi a $Z$. Gardneri differt: aculeis geminis patentibus, floribus villoso-tomentosis, foliis plerumque obtusis et subtus pubescentibus, nec prominulo reticulatis, - Etiam patria species nostra ab affinibus diversa.

35. Sulene thysandedes Fenzl. S. perennis humilis pauciflora ; foliis oblong 0 - lanceolatis ( $1 \frac{113-2}{2}$ "lg.) uninerviis utrinque glabris marginibus pube crispa molli densissime barbatis, calyce inflato amplo campanulato $(1 / 2 " \lg$.$) glanduloso - tomentoso$ quinquefido, laciniis ovatis obtusiusculis, petalorum calycem subsuperantium lamina erecta truncata subquadrata, ungue latiore ofovato cuneato-attenuato plus triplo breviore, fauce lamellis duabus auriculaeformibus subfornicatis discretis obsoletissimis coronata.

Patria: America aequatorialis occidentalis. Hab. ad radices montis ignivomi Colopaxi, reipublicae Ecuador, alt. 12000' (Francis Hall! in Herbar. Kunth.)

Species cognitarum nulli propius affinis, in sectione Betieñantha $D C$. collocanda. Habitus fere Silenes pumilio, ast parum praccocior, nec strenue uniflora, vix dense caespitans. hadix non visa. Caudex simplicissi. mus, haud lignosus, crassitie fere calami scriptorii, vaginis foliorum emarcidorum inferius tunicatus, superius rosula foliorum plurimorum densa coronatus, cauliculum crassitie fili emporetici mediocris digitalem, erectum, simplicissimum, inferius aphyllum, circa apicem foliorum bractealium paribus duobus instructum, triflorum (saltem e gemmis floralibus flore terminali solitario subjectis dijudicatum), albido-tomentosum emittens. Folia infima congesta, oblongo-lanceolata, acutiuscula, mutica, evoluta plurimum biuncialia v. parum breviora, supra basim vaginantem attenuatam reflexa, rosulatim disposita, juniora breviora, intus plurimum canaliculato-convoluta, crecto-patula, omnia utrinque glabra, uninervia, marginibus pube geniculata crispa, albida, molli, semilineam longa, a 
basi usque ad apicem aequabiliter densa elegantissime fimbriato-barbata; p raeter folia bractealia semiuncialia ac sensim breviora, lanceolata, ad a picem nervumique medium postice. villosula, caulina nulla. Flores axilla$r$ es et alares, paucissimi (terminali excepto reliqui forsan abortientes), flosc.ulo infimo axillari sessili bibracteato posthume efforescenti typum in:florescentiae compositae cymoso-paniculatae obsoletae exhibentes, mole - culycis inflato campanulati, semiunciam longi et $4^{\prime \prime \prime}$ lati, densissime glan-duloso-tomentosi, semiquinquefidi insignes, pedicello dimidio breviore insidentes, subnutantes; calycis laciniae marginibus barbatae, inferius latissime lineares, apicem versus formam ovatam obtusinsculam induentes, purpurascentes visae. Petalorum calycem vix superantium lamina erecta, subquadrata, angulis rotundata, marginibus apiceque truncato integro eroso-crenulata, in sicco flavescens, fauce lamellis duabus obsoletissimis, sinu lato discretis, auriculaeformi-fornicatis coronata, ungue latiore obovato, ad insertionem laminae utrinque in lobulum ovatum, brevissinum erectum producto, hinc facis fere obcordato, cuncato-attenuato, trinervato, plus triplo brevior. Stamina 10, subulata, petalis dimidio breviora. Styli tres cylindrici, staminibus sub anthesi aequilongi. Ovarium infra medium perfecte, apicem versus imperfecte triloculare, carpophoro distincto, crasso stipitatum. Capsula et semina matura non visa.

40. Thonasia macrocarpa Hügel msc. Th. foliis ovatis cordatis angulato-dentatis supra hirtis subtus hirsuto-tomentosis, petalis nullis, staminibus decem, alternis sterilibus, ovarii loculis biovulatis.

\section{Colitur in horto Hügeliano.}

Suffrutc $x$ in specimine culto orgyalis, canle ramisque teretibus, tomentosis, pilis stellatis internixtis hispido. Folia alterna, petiolata, petiolo pollicari, densissime hispido - tomentoso, ovata, acuta, basi cordata, duos pollices longa, sesquipollicem lata, angulato-dentata, nec sinuata, supra hirta, adultiora subglabrata, subtus dense hispido-tomentosa, trinervia. Stipulae sessiles, ohlongae, dimidiato-cordatae, tres lineas longae, Jatere exteriore prope basim subincisae. Racemi oppositifolii, bipollicares, tri-quinqueflori. Bracteae hypocalycinae trifidae, calycem aequantes. Calyx extus pilis stellatis hispidulus, pallide lilacinus, expansus diametri semipollicaris. Stamina decem, alterna ananthera, paullo longiora, conniventia, fertilium antherae atropurpureae. Ovarium dense tomentosum, stylo stamina paullo superante, ima basi hirto, apice glaberrimo, intus trílciculare. Ovula in loculis constanter gemina, collateralia, ex anguli centralis basi adscendentia. 
Nr. 5.

\section{NOVARUII STIRPIUII DECA D E S}

\section{Watae a Museo Caesareo palatino Vindobonensl.}

Nr. 5. Vindobonae, typis Sollingerianis. 1. Juni 1839.

COELOSTYLIS Torr. et A. Gray msc. Endl. Gen. plant. Supplem. $n . \frac{3363}{1}$. Calyx quinquepartitus. Corolla hypogyna, infundibuliformi-campanulata, fauce nuda, limbi quinquepartiti laciniis aestivatione valvatis. Stamina 5 , medio corollae tubo inserta, inclusa. Ovarium biloculare. Ovula in placentis septo utrinque insertis plurima, amphitropa. Stylus simplex, infra medium articulatus, articulo superiore cavo. Stigma fusiformi subclavatum, pilosum, apice glanduloso nudo, subemarginato. Capsula .....Herba floridana; caule tetragono, foliis oppositis, integerrimis, stipulis interpetiolaribus liberis, persistentibus, floribus axillaribus, subsolitariis.

Genus Loganiaceum, prima facie Loganiis sectionis Stomandra proximum, revera ob corollae aestivationem valvatam, cum fructu certissime capsulari polyspermo combinatam, distinctissimum, propriam in ordine multiformi tribum sibi vindicans. Fructus pauca rudimenta in specimine unico supersunt, quae capsularem ejus naturam tamen evincunt, et suspicionem movent, valvas angusto margine introflexas post dehiscentiam mox delabi, placentas vero juxta axim unitas diutilis persistere, quo charactere Geniostomati arctius accederet novum hocce genus, Euloganieas in Strychnearum nostro subordine repraesentans, a Spigeliaceis, quibus stylo articulato accedit, ovarii structura alienum.

4. Coelostylis Ioganioides Torr, et A. Gray msc. Endl. Iconographia Gen. plant. t. 101.

Habitat in Florida occidentali, prope Tampa Bay. (Dr. Burrows et Mr. Alden in Herb. Torrey.)

Specimen, quod video unicum, est ramus v. fortassis caulis, sesquipede paullo longior, simplicissimus, tenellus, tetragonus, glaberrimus, ad angulos, e foliorum basi decurrente prominulos, sub lente valde augente subtilissime serrulatus, tactu laevis, parte inferiore foliorum delapsu audus, stipulis persistentibus internodia circiter pollicaria indicantibus, 
supra medium opposite foliosus, foliorum paribus internodiis dimidio pollice paullo brevioribus sejunctis, versus apicem tandem approximatis. Folia opposita, ovalia v. elliptica, obtusa, basi angustata, in petiolum brevissimum attenuata, quatuor ad novem lineas longa, tres v. quatuor lineas lata, subcoriacea, margine subrevoluta, integerrima, glabra, supra ex sicco saturate viridia, subtus pallida, subglaucescentia, trinervia, nervis pagina superiore impressis, inferiore prominentibus, medio validiore, secundarios paucos alternos, apice confluentes, angulo acuto exserente, lateralibus simplicibus intra marginem desinentibus. Stipulae interpetiolares lanceolatae, acutae, dimidia linea paullo longiores, cum ima petiolorum basi persistentes. Flores in axillis foliorum superiorum solitarii, alterni, in supremo foliorum pari gemini, quorum unus praecocior subsessilis, alter basi longius pedicellatus. Pedicellus unius fructus rudimentis onustus, in axilla folii tres lineas longus, floris alterius in apice rami condum evoluti sesquilinea longior, floris expansi dimidia linea brevior, omnes basi bractea minima squamaeformi, bracteolisque duabus, altera pedicello supra medium inserta, altera calyci admota instructi. Calyx fere ad basim quinquepartitus, laciniis lineari-lanceolatis, acutis, uninerviis, sub lente margine subtilissime serrulatis, sesquilinea paullo longioribus, erectiusculis, fructiferis reflexis. Corolla hypogyna, infundibuliformis, quatuor lineas longa, tubo inferne calycis longitudine angusto, superne sensim ampliato, limbi quinquepartiti laciniis lanceolatis, acutis, aestivatione marginibus subincrassatis valvatim commissis, sub anthesi patentibus, longitudinaliter venosis, cum tubo fauceque glaberrimis. Stamina 5 , medio corollae tubo inserta, ejustem laciniis alterna, inclnsa, aequalia; filamenta teretiuscula, antherae oblongae, basi subbifidae, erectae, apice obtusae, biloculares, loculis appositis, introrsum juxta totam longitudinem dehiscentibus. Ovarium ovatum, glaberrimum, dimidio calyce brevius, superne in stylum angustatum, intus biloculare, placentis hemisphaericis, linea dorsali medio dissepimento insertis. Ovula plurima, dense compacta, amphitropa. Stylus cylindricus, corollae faucem aequans, gla.berrimus, infra medium articulatus, articulo inferiore ovario concolore, solido, superiore cavo, pellucido. Stigma terminale, incrassatum, fusiformi subclavatum, pilis brevissinis flavescentibus hirtum, juxta longitudinem bisulcum, apice attenuato brevissimo nudum, papillam glandulosam, nitidam, emarginatam exserens. Fructus fragmentum exhibet calycem reflexum, valvarum delapsarum imam basim, et placentae centralis rudimentum.

APHANOPETALUM Endlicher Gen. plant. n. 4650. Calyx tubo subnullo, ibero, limbi explanati, quadripartiti lobis membranaceis, venosis, subinaequalibus. Corollae petala 4 , inter calycis lacinias inserta, lineari-lanceolata, minima, saepissime nulla. Stamina octo, imo calyci inserta; filamenta filiformi-subulata, antherae biloculares, basifixae, subsagitlatae, ecaudatae. Ovarium liberum, quadriloculare, loculis ovulo unico, ex apice anguli centralis pendulo, uno alterove effoeto. Styli 4 , in unicum quadripartibilem cohaerentes; stigmata brevia, acuta, stellatim patentia. Fructus.... - Arbores Novae-Hollandiae orientalis; ramulis verruculosis, foliis oppositis, breve petiolatis, ovatis, nitidis, serratis, stipulis interpetiolaribus caducis, paniculis axillaribus lateralibusque, pedicellis medio setaceo-bibracteolatis. 


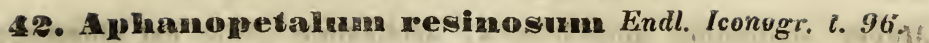

Habitat in Nova-Hollandia orientali extratropict. (Hert. Bauer, A. Cunningham. Hügel.)

Ramuli teretes, pennae anserinae crassitie, cortice rabro-fusco, verruculis plurimis exasperato, glaberrimo, nitido, versus summitates subresinoso vestiti. Folia opposita, internodiis inter paria bipollicaribus, breve petiolata, ovato-elliptiea, duos v-tres pollices longa, pollicem v. sesquipollicem lata, basi saepius subcuneata, apice angustata, acuta v. obtusiuscula, remote et obtuse serrata, coriacea, glaberrima, supra resinoso nitida, utrinque reticulato-nervosa, venosa, petiolo vix duas lineas: longo, subtirs convexo, supra canaliculato, in vaginam siiputarem brevissimam, caducain, cicatricem annularem circa nodum nonnihil fincrassatum reTiquentem commissi. Paniculae axillares, rarius laterales, simplices v. compositae, falio breviores. Peclicelli rhachisque generalis tetragono-filiformis. Bracteae ad basim pedicellorum squamiformes, lineam longae. Bracteolae mediis pedicellis insertae, setaceae, deciduae. Calyx tubi libero, limbi explanati quadripartiti laciniis 5 lineas Iongis, duas circiter lineas latis, obtusis, membranaceis, venosis, glabris, plernumque una altera paullo longiure. Petala lineari-lanceolata, brevissima, in paucissimis floribus omnia praesentia, in plurimis nulla. Stamine vix dimidiis calycis laciniis aequilonga, stylo breviora. Ovarii lacali ra. rissime omnes perfecti, plernmque unims v. dao nani, efoeti.

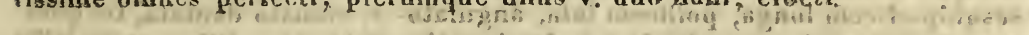

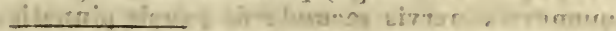

XEROPETALUII Delile. Calyx quinquepartitus, persistens, nudus. Corollae petala quinque, imo calyci inserta, inaequilatera, aestivatione convoluta, tanden scariosa, persiscentia. Stamina 20 , cum petalis inserta, basi brevissime monadelpha, quirque sterilia ligulaeformi-subclavata tribus fertilibnts interjecta; lilamenta filiformia, antherae terminales, linéres, biloculares, lacere juxia totam longitudinem deloiscentes. Ovarium sessile, densé villosum, tri-quinqueloculare. Avula in loculis: $2^{2} 3$ collateralia, ex anguli centralis basi adscendentia, anatropa. Stylus terninnitis, cylindricus, tri-guirquefidus v. partitus; stigmatities acutis, revolutis. Capsula coriacea, hirsuta, tri-quinquelacularis, loculicide tri-quinquevalvis, valvis medio septa ab axi seminifera-nerviformi persistente soluta gerentibus. Semina in loculis solitaria, adscendentia, ovata, subangulates, testa coriaceco, ad chalazan rpicalem lutam incrassata, rhaphe inirorsa, sotubili. Eimbryo intra albumen carnoso-mucilaginosum orthotropus; colyledonibus foliaceis, bipartitis, suliconvalutis, radicula brevi, infera. bores africanae, floresculiat tempore saepe aphyllae; folios al ternis, petiolatis, integris, basi cordatis, dentatis vo crenatis, stipulis decidnis, Rorum paniculis lateralibas, pediceltis carymbosis v. unbellatis, bracteolatis, bracteolis deciduis, interdum alabastro approximatis, nec tamen involucellum verun formantibus...........

Genus a clarissimo Delile CCenlurie de plantes d'A frique: du Voyage it Mrroé, recueillies pur M. Cailliaur. "Paris', 1826. 8 . 7. 84.7 constitntum, et ah codem Tiliaccis adnmeintiin, cui pratfer species duas africanas ineditas tertiam capongemal nugup inter

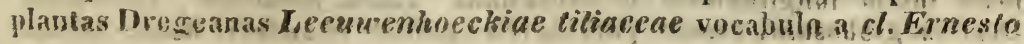


Meyer evulgatam adscribimus, arctissime affine est Dombeyae et Melhaniae, generibus accuratius circumscribendis et repurgandis.

43. Xeropetalum multiforum Endl. $\mathbf{X}$. foliis supra scabris subtus tomentosis, petiolis pedunculisque corymbosis calycibusque glaberrimis, stylo tri-quinquepartito.

In Africae regionibus Shangub et Camamil legit cl. Theodorus Kotschy. (n. 656. et 685.)

Arbor haud celsa, florescentiae tempore fere aphylla. Rami digitum minimum crassi, basi torosi, superne teretes, laeves, cortice sordide cinereo, crasso, tenaci vestiti, ramulos alternos, patentiusculos, paullo tenuiores exserentes, pollicis unius intervallis foliorum delapsorum cicatricibus oblongo-semiovatis, spirali serie circumeuntibus notati. Gemmae supra cicatrices foliorum delapsorum multisquamosae, in superiore ramulorumque parte paucae, mere foliiferae, in inferiore foliiferae, vo nonnullae inflorescentiae ramis foliis nonnullis munitis, interdum mixtae. $R a$ muli foliiferi fasciculati, in speciminibus a me examinatis plerumque in quavis gemma unicus, reliquis adhuc brevissimis, vixdum folia explicantibus, rite evolutus, pollicaris v. paullo longior. Folia alterna, longe petiolata, ovata, basi cordata, apice obtuso v. acuminato-obtuso, maxima sesquipollicem longa, pollicem lata, angulato- $v$. sinuato-dentata, tri-quinquenervia, nervis secundariis paucis pinnatis, apice bipartitis, supra pilis stellatis raris, in adultioribus verisimiliter evanidis scabriuscula, subtus confertioribus, quidquam longioribus cinereo-subtomentosa. Petioli quatuor ad sex lineas longi, glaberrimi, laevissimi, semiteretes, dorso convexi, facie canaliculati, basi dilatati, gemmam villosam in axilla foventes. Stipulac petiolorum basim utrinque stipantes subcoriaceae, lanceolatae, acutae, duas lineas longae, apice barbatae, caeterum glabrae. Ramuli floriferi ex inferiore ramorum parte orti, fasciculati, tri-sexpollicares, ramosi, glaberrimi, laeves, inferne teretes, superne plus minus compressi, nunc omnino aphylli, nunc ex uno alterove ramulo folia is ramulorum foliiferorum conformia, sed minora explicantes, basi simplices et apice di-trichotome ramosi, paniculati, v. statim a basi ramosi; pedicelli corymbosi, tres ad sex lineas longitudine metientes, filiformi-subcompressi, glaberrimi, praeter bracteam minutam, bracteolis conformibus $2-4$, alternis, interdum alabastro admotis, linearibus, acutis v. obtusis, carinatis, apice pilosis, dimidia linea brevioribus, v. linea paullo longioribus stipati. Alabastra ovata, basi obtusissima, apice subacuta, glaberrima, viridia, prope basim purpurascentia. Caly $x$ tres et dimidiam lineam longus, quinquepartitus, laciniis lanceolatis, acutis, lineam latis, obsolete trinerviis, glaberrimis, basi fere ad unius lineae longitudinem connatis, aestivatione valvatis, sub anthesi supra basin arcte reflexis. Corollae petala 5, perigyna, imo calyci inserta, ejusdem laciniis alterna, aestivatione convoluta, sub anthesi erecto-patentia, oblongo-cuneata, sex lineas longa, apice tres lineas lata, oblique subtruncata, integerrima v. obsolete lerosa, inaequilatera, primum membranacea, pallide rosea, nitida, pellucida, mox seariosa, rigidiuscula, venis flabellatis, basi simplicibus, apice ramosis, reticulatim anastomosantibus, primum tenellis, mox incrassatis percursa, persistentia. Stamina perigyna, cum petalis inserta, persistentia, quindecim fertilia, alterna breviora, sterilia quinque inter tria fertilia sita, fertilibus longiora, calycis foliolis opposita, clavato-ligulata, apice sub granulosa, omnia brevissime monadelpha. Staminum fertilium filamenta filiformia, glabra, lineam longa v. paullo breviora. Antherae terminales, biloculares, loculis linearibus, subcartilagineis, connectivo angustissimo in apiculum obtusiusculum brevem excurrente ntrinque adnatis, latere juxta totam longitudinem dehiscentibus. Pollen globosum, echinatum Ovarium globosum, sessile, pilis rigidis, pallide stramineis dense hirtum, 
tri-quinqueloculare, loculo uno alterove saepissime minori sterili. Ovula in loculis $2-3$, in angulo centrali paullo supra basim collateralia, adscendentia, anatropa, rhaphe introrsa. Stylus una linea paullo longior, pilosus, profunde tri-quinquepartitus, laciniis linearibus, obtusis, intus canaliculatis, primum conniventim erectis, mox revolutis. Fructus globosus, Pisi majoris mole, petalis staminibusque persistentibus stipatus, stylo superatus, dense hirtus, tri-quinquelocularis, epicarpio coriaceo secedente, endocarpio tenuissime membranaceo, loculicide tri-quinquevalvi, valvis medio septa a columella tenuissima nerviformi secedentia gerentibus. Semina in loculis abortu solitaria, e columellae basi adscendentia, testa coriacea, ad chalazam terminalem latam incrassata, rhaphe introrsa, solubili. Endopleura carnosa. Albumen mucilaginosum. Embryo intra albumen orthotropus, viridis; cotyledonibus bipartitis, lobis divaricatis, obtusis, subconvolutis, radicula brevi, crassiuscula, infera.

OBSERVATIO. Xeropetalum quinquesetum Del. J.c. a nostro floribus multo majoribus, et inflorescentia diversum videtur.

44. Xeropetalumn mainens Endl. X. foliis supra scabris subtus tomentosis, petiolis pedunculisque corymbosis glaberrimis, calycibus basi hirtis, stylo tri-quinquepartito. .

\section{Cum praecedente legit cl. Theodorus Kotschy. (N. 525.)}

Species a praecedente floribus minoribus et calyce basi hirto prima facie distincta. Ramulos video nonnisi florentes teneros, semipedales, paniculatim ramosos, ramis strictis, glaberrimis, laevibus, corymboso-multifloros. Folia ut in praecedente specie, paullo majora, obtuse dentata, pilis densioribus vestita. Flores minores. Alabastra ovata, basi hirta, apice barbata. Calyx reflexus, basi hirtus. Petala paullo magis inaequilatera, apice magis truncata et quasi emarginata. Stamina fertilia subaequilonga, sterilia fertilibus triente longiora. Ovarium hirtum, tri-quinqueloculare. Stylus tri-quinquepartitus, laciniis revolutis.

45. Xeropecalum tiliaceum Endl. X. foliis supra vix scabris subtus glabris, petiolis pedunculis bifidis bifloris calycibusque iomentosis.

\section{Leeuventoeckia liliacea Ern. Meyer msc.}

\section{In Capite Bonae Spei legit cl. Drège.}

Rami teretcs, pennae scriptoriae crassitie, cortice rubicundo, lenticellis crebris foliorumque delapsorum cicatricibus notato. Ramuli sparsi bi-quadripollicares, patentes, inferne foliorum annotinorum cicatricibus torosi, apice foliosi. Folia alterna, longe petiolata, ovato-oblonga, obtusa, basi subcorlata, sesquipollicem longa, unum pollicem lata, rotundato-crenata, nervis primariis tribus, secundariis pinnatis, venis reticulatis, supra scabriuscula, subtus glabra. Petioli octo lineas longi, dorso convexi, facie subcanaliculati, dense rinereo tomentosi. Stipulae in speciminibus observatis jan delapsae, sed earum cicatrices ad hasin petio. lorum nonnihil incrassatau transversim oblongae. Pedunculi in axillis foliorum solitarii, sesquipollice paullo longiores, ad mediun divaricato-bifili, ramis unilloris, tomento eodem quo petioli induti. Calycis quinquepartiti laciniae lancenlatae, acuminatac, quatuor lineas longae, 
lineam latae, cxtus dense tomentosae, intus glabrae, arcte reflexae. $P e$ tala subscariosa, ex sicco flava, obovata, inaequilatera, subtruncata, quatuor et dimidiam lineam longa, quatuor lineas late. Stamina congenerum, filamentis paullo longius monadelphis, sterilibus fertilia longitudine dimidio superantibus. Ovarium hirtum, quinqueloculare, loculis triovulatis. Stylus filiformis, petala longitudine aequans, apice breviter quinquefidus, laciniis acutis, revolutis. F'ructus desideratur.

RUSSEGGERA Endlicher Gen. plant. Suppl. n. $\frac{4072}{1}$. Calyx ait basim quinquepartitus, laciniis lateralibus paullo latioribus. Corolla hypogyna, ringens, labio superiore erecto, subfornicalo, emarginato, inferioris tripartiti, patentis laciniis subaequalibus, obtusis. Stamina 4, corollae tubo inserta, inclusa, didynama, antherae biloculares, loculis insertione inaequalibus, obtusis, margine ciliolatis. Ovarium biloculare, loculis uniovulatis. Stylus simplex; stigma obtusum. Capsula chartacea, rostrata, bilocularis, disperma, septo ad parietes incrassato, ad axinu sulco interrupto rostroque incrassato cartilagineis, loculicide bivalvis, valris mox bipartitis. Semina compressa, retinaculis late cymbiformibus obtusis subtenta, testa humectata in vasa spiralia soluta. _. Fruticulus depressus; caudiculis lignosis, fasciculatim ramosis, ramis squamis coriaceis imbricatis, foliis fasciculatis, lineari-lanceolatis, acuminatis, submucronatis, pilis mollibus candicantibus, pedunculis axillaribus solitariis, unifloris, bibracteolatis.

Genus Acanthaceum, nulli cognitorum arctius affine, habitu et characteribns distinctissimum, dixi in honorem Josephi Russegger, viri inter nostrates itineribus per Asiam et Africam longe celeberrimi, cujus ductu Theodorus Kotschy regiones botanicis autea inaccessas feliciter penetravit, et cujus industriae, non tantum metallorum venis in remotis illis plagis indagandis, sed scientiis naturalibus ¿quoquo modo provehendis intentae, praeter alias ipsam hanc stirpen in acceptis referimus.

4. Russeggera collima Endl. Iconogr. Gen, pl. t. 9 t.

In collibus Gebbel Accara juxta fluvium Tumad legit cl. Rusagger.

Caudiculi pollicares sesquipollicaresque, simplices v. unoalterove ramulo aucti, pennae anserinae crassitie, lignosi, cortice tenaci, sordide bruneo, lacero vestiti, apice in ramulos plurimos semipollicares, fasciculatos, dense eapitatim congestos divisi, ramuli squamis coriaceo-snbscariosis vestiti. Squamae sordide bruneae, ovatae v. oblongae, acutae v. obtususculae, uninerves, obsolete longitudinaliter striatae, inferiores tres $\mathbf{v}$. quatuor lineas longae, tres lineas latae, superiores magis elongatae, pallidiores, summae lineari-lanceolatae, scariosae, sex lineas longae, duas lineas latae, flores solitarios v. densos foliorum fasciculos in axillis foventes. Folic fasciculata, lineari-lancedata, acuminata, submucronata, apicibus subrecurva, sex lineas longa, unam lineam lata, subtrinervia, glauco-virid.d, pilis albis, sallihus, candicantibns, patentibus, juxta marginem subrectrorsis, utrinque imprimis subtus villosa. J'edunculi in squamarum axillis colitarii, Urevisimi, basi antice bravtea, apice ntrin- 
yre bracteolis geminis stipati. Bractea 4 lineas, bracteolaeque sex lineas longitudine metientes, vix lineam latitudine sua superantes, lanceolato. acuminatae, demum scariosae, uninerves, apice pilis mollibus retrorsis hispidae, erectae, calyci adpressae. Calyx fere ad basim quinquepartitus; foliola lanceolato-acuminata, erecta, basi incrassata subcarinata, trinervia, apice patentiuscula, pilis albis mollibus candicantia, demum subscariosa, omnia aequilonga, sex lineas metientia, inaequilata, duo lateralia exte. riora duas lineas, duo antica et posticum unam linam lata. Corolla quinque lineas longa, ringens, ex sicco flava. Tubus limbo quidquam brevior, quinquenervis, cylindricus, sursnm sensim ampliatus, ad faucem nonnihil constrictus, extus glaber, intus basi infra staminum insertionem pilorum nitentiun fasciculis retrorsis hispidus. Limbi bilabiati labium superius erectum, obovatum, obtusum, breviter emarginatum, subfornicatum, longitudinaliter venosum, inter venas maculis subquadratis, in sicco nigricantibus, in planta viva verisimiliter purpureis pictum, juxta venas pilis retrorsis hispidulum, intus prope faucen linea brevi, pilis retrorsis nitentibus hispida, staminis postici deficientis locum indicante notatum. Labium inferius tripartitum, lobis labium superius aequantibus, inter se aequilongis, intermedio nonnihil latiore, omnibus patentibus, obtusis, trinerviis, ad nervos et juxta margines pilis patentibus raris conspersis. Stamina 4, didynama, corollae tubo paullo infra medium inserta, dno postica inter corollae labium superius et inferioris lobos laterales inserta, antherarum apices breviter e fauce exserentia, duo antica inter labii inferioris lobum medium et laterales sita breviora, faucem aequantia. Filamenta filiformia, teretiuscula, glabra. Antherae biloculares, loculi oblongi, obtusi, insertione inaequales, altero sursum, altero deorsum longius prominente, longitudinaliter dehiscentes, valvulis subcartilagineis, ad margines breviter ciliolatis. Ovarium ovatum, brevissime substipitatum, a latere compressum, vix lineam longum, apice acuto incrassatum, biloculare, septo juxta ovarii parietes crassissimo, versus centrum cuneatim angustato. Ovula in loculis solitaria, e squamula brevissima septi basim juxta axim in uno loculo hine, in altero illinc marginante erecta. Stylus terminalis, filiformis, glaber, staminibus majoribus paullo longior. Stigma terminale, truncatum, subemarginatum. Capsula calyce parum aucto inclusa, ovata, compressa, rostrata, quatuor lineas longa, duas lineas lata, bilocularis, septo cartilagineo ad parietem incrassato, lineam fere lato, versus axim sulco interruptum angustato, in rostrum aequilatum, lineam longum continuo, valvarum parietibus tennissine chartaceis, loculicide subelastice bivalvis, valvis mox septicide bifidis. Semina in loculis solitaria, ovata, compressa, basi subcordata, apice oltusa, duas lineas longa, e retinaculis cartilagincis, late cymbiformibus, semiseptorum imo margini alternatim lata basi insertis, obtusis, medio foramine, cui embryonis radicula admota, pertusis erecta, sicca integumento fusco rubro, ad speciem cartilagineo, ad margines et dorso juxta lineau mediam pallidiore, apice fnlvo, quasi lacero, humectato in vasa spiralia innumera, nitida, longitudine sua seminis diametrum excedentia cum impetu soluta, vasis patentibus, juxta margines adscendentibus, juxta dorsi lineam mediam biseriatim retrorsis. Embryo exalbuninosus, seminis integumento interiore adhaerente tunicatus; coiyledonibus cordatis, dorso convexis, facie planis, radicula tereti, versus capsulae centrum incurva, racdium retinaculi foramen spectante.

PLÖSSLEA Endl. Calyx quinquefidus. Corollae petala 5, disco extus inserta, aequalia, ungue nudo. Stamina 10 , cum petalis inserta; filamenta subulata, antherae longiludinaliter dehiscentes. Discus completus, integerrimus, ovarii basim cingens. Ovarium 
criloculare. Ovula in loculis gemina, angulo centrali inserta, oblique contigua, peltatim amphitropa, micropyle supera. Stylus terminalis, crassus; stigma depresse subcapitatum, obsolete trilobum. Capsula clavata, trilocularis, trivalvis, valvis cum septis alternantibus, columnan tripteram, alis septantem, faciebus seminiferam nudantibus. Semina in loculis solitaria, triangularia, basi ec apice in cuspidem producta, umbilico ventrali, testa subossca, dorso membrana arillari lacerula tecta. Nucleus testa multo minor, integumento tenuissimo. Embryonis exalbuminosi, leviter arcuati cotyledones foliaceae, trilobae, convolutae, radicula supera. - Arbor Africae tropicae; foliis ignotis, sub florum anthesi verosimiliter nullis, paniculis in apice ramorum confertis, multifloris.

Genus Sapindaceum, nulli contribulium arctius affine, staminibus cum petalis disco extus insertis, ejusdem disci basi cum calyce cohaesione, unde organorum floralium insertio quasi perigyna, fructus fabrica et seminis structura distinctissimum, et in ordine anomalum, benemeritis viri modestissimi honoribus pro virili consulturus dicavi Simoni Plössl nostrati, inter artifices opticos ingenio, dexteritate et industria nulli secundo, cujus microscopiis egregia arte constructis plurimi botanicorum, non sine maximo scientiae nostrae emolumento, laeti utuntur.

\section{4\%. PIdisslea floribunda Endl.}

Crescit in Africae tropicae orientalis regione Fa\%oglou, ubi florentem et fructiferam, sed aphyllam legic Theodorus Kotschy. (n. 455.)

Rami floriferi et fructiferi quos video omnino aphylli, alii digiti minimi crassitie, paniculis brevioribus, alii indicis circumferentia, paniculis longioribus laxioribusque terminati, omnes ligno molli, albido, cortice pallide stramineo v. rubro-fusco, crassiusculo, tenaci, rugosissimo, rimoso, foliorum delapsorum cicatricibus late triangularibus confertim sparsis toroso, hinc inde, imprimis prope apicem rami exsudationibus gummosis, ad petiolorum cicatrices saepius in guttas validas induratis conspurcato. Paniculae e gemma ramum terminante, cujus squamae cicatricibus transversim oblongis indicantur, quinque ad duodecim, omnes prima facie ex eodem plano ortae, simplices, ramorum teneriorum tres pollices longae, validiorum pedem etiam longitudine sua superantes, strictiusculae v. exteriores laxiuscule patentes, nonnunquam fere divaricatae. Panicularum axis primarius penna corvina paullo tenerior, praeter basim nonnihil latiorem, dorso convexam, facie subcomplanata obsolete canaliculatam teres, ramulique alterni, inferiores bi-tripollicares, superiores vix pollicem longitudine sua superantes, sordide virides, aut in nonnullis speciminibus rubescentes, pube conferta, brevi, rigidula, sordide fuscescente $v$. cinerascente vestiti, hinc inde guttulis resinosis notati, basi bractea brevissima, saepe fere obsoleta instructi. Pedicelli alterni, sesquilineam longi, glaberrimi, sursum nonnihil incrassati, ante anthesim nutantes, sub anthesi horizontaliter patentes, basi bractea vix dimidiam lineam longa, paullo supra basim bracteolis geminis, minutissimis, antrorsum sublateralibus stipati. Calyx glaberrimus, ferme cupularis, lineam longus, explanatus sesquilineam metiens, quinquefidus, lobis late triangularibus, "brevibus, obtusis, aestivatione anguste imbricatis. Discus carnosus, annularis, integerrimus, ima basi calyci inferne adnatus, ovarii basim laxiuscule cingens. Corollae petala 5 , disci basi extus inserta, ealycis lasiniis elterna, inter se aequalia, ovato-lanceo- 
lata, acuta, aestivatione marginibus sese imbricatim tegentia, sab anthesi patentia, duas et dimidiam lineam longa, una linea paullo latiora, obsolete uninervia, reticulatim venosa, ungue nudo, paullo obscurius colorato, caeterum glaberrima, integerrima, quantum in sicco video rosea, plus minusve pallida, plurimorum florum in nostris speciminibus omnino decolora. Stamina decem, disci basi extus cum petalis inserta, iisdem per vices alterna et opposita, brevia; filamenta complanato-subulata, dimidia linea paullo longiora, discum triente superantia, eidem adpressa, inter se aequalia, $v$. postica in nonnullis floribus paullo longiora, - sub anthesi stricta v. laxiuscule patentia, antheris delapsis diu persistenlia. Antherae ovato-oblongae, obtusae, biloculares, luteae, flamentis parum breviores, basi emarginata insertae, subversatiles et facile deciduae, staminum anticorum in plurimis floribus quidquam minores, omnes loculis suboppositis, in alabastro marginibus involutis subbilocellatis, basi et apice obtusis, superne contiguis, inferne subdivergentibus, juxta to tam longitudinem dehiscentibus. Pollinis lutei granula globosa, triedra. Ovarium brevissime stipitatum, subglobosum, superne in stylum nicum, crassum, triquetrum, cavitate triente longiorem, stamina paullo superantem continue transiens, intus triloculare, rarissime quadriloculare, loculo uno tunc efocto v. saltim multo minore, extus undique glabrum. Stigma in apice styli subconvexum, papillosum, ambitu obsolete trilobum. Ovula in loculis gemina, angulo centrali peltatim inserta, oblique superposita, ita ut prima fronte collateralia videantur, alterum inferius multoties minus, cito obliteratum, superioris inferne rotundati parte superiore longius producta, sursum micropyle aperta. Capsula clavata, obtuse triquetra, ima basi calycis reliquiis et disco persistente indurato, nihilum aucto stipata, trilocularis v. rarissime quadrilocularis, loculo uno tunc minore, efoeto $v$. semen inane fovente, octo v. novem lineas longa, ima basi faciebus planis, vix lineam latis, a basi ad quatuor linearum altitudinem in trium linearum latitudinem sensim dilatatis, inde mox tres et dinidiam lineam latis, convexis et semine turgidulis, mox iterum angustatis, apice obtuso, vix sesquilineam latitudine sua aequante. Valvulae tres, aut ra. rissime quatuor, a columna triptera, rarissime imperfecte tetraptera, alis cum valvis alternantibus septa constituenie, faciebus seminifera, maturitate primum basi et apice, mox undique solutae, rigide coriaceae $\mathbf{v}$. fere lignosae, extus in sicco sordide violaceae, subrugosae, intus sordide stramineae, fere planae, nonnisi infra apicem obtusum, ubi ex illa*um regione semen situm est turgidulae, ejusque pressione intus excavatae, extus convexiusculae. Columna centralis tenuiter lignosa, triptera, apice in stylum brevissimum, vix dimidiam lineam longum, teretem, tenuem, continum et persistentem abruptim desinens, alis dissepimenta valvarum margines contingentia constituentibus, latitudine maxima bilinearibus, deorsum sursumque sensim angustatis, ex axi vasis paucis percursa, vix crassiore, angulo obtuso continuo productis, facies seminiferas, obtusissime concavas, valvis oppositas praebentibus, puncto insertionis seminis, sestertiae lineae distantia infra apicem sito, ovuli secundi rudimento interdum immediatim sublateraliter infra illud conspicuo, saepissime nullo. Semen triquetrum, tres lineas longum, sestertiam lineam latum, dorso subrotundatum, apice in acumen, lineam longum, fere ad apicem columnae centralis pertingens, eique adpressissimum productum, marginibus acutum, ibique et dorso membrana laniellari lacerula (arillo exsiccato?) tectum, facie laeve, vix convexiusculum, apice in acumen dorsale continuum, baseos truncatae, in dorsum convexe continuatae medio in acumen subulatum, rigidum, dimidian lineam vix superans desinens, Testa fere ossea, pallide straminea, dorso rugosa, facie laevis, ibique paullo infra medium umbilicum brevem, linearem, subimpressum, obscurius coloratum exhibens, micropyle punctiformi, imınediatim supra umbílici extremitatem supcrioren sita. Nuclcus testae cavitate, intus laevigata multo minor, parvae ejusdem protuberantiae (umbilico) peltatim insortus, compressus, mesubrana tenui, transparente vestitus. Albumen 
nullum. Embryo viridis, leviter arcuatus. Cotyledones late ovatae, trilobae, lobis acutiusculis, intermedio paullo longiore, lateralibus bifidis, laciniis exterioribus nonnihil brevioribus, alterius, seminis dorsum respicientis, cotyledonem interiorem marginibus amplexantibus et involventibus. Radicula brevis, subcylindrica, nonnihil versus seminis faciem incurva, fructus verticem spectans.

SEMONVILLEA Gay. Calyx usque ad basim5-particus, sepalis ovalibus oblongisve, medin herbaceis carnosulis, margine membranaceis. Petala 5 v. nulla, lamina subrotunda, integerrima, in unguem angustum rursus coarctata. Stamina 7 hypogyna; eorunb 5 petalis, 2 sepalis anteposita; filamentis e basi ovata, maryine, fimbriata subulatis. Antherae versatiles. Ovarium 2-, abortu subinde 1-loculare, 2-ovulatum, lenticulari-compressum; ovulis placentae basilari dorso carpophylli semiadnatae affixis, campylotropis; stylis 2 filiformibus, intus stigmatosis, dissepimento situ contrariis. Fructus dicarpellaris, carpidiis crustaceis orjiformibus, faciebus commissuralibus contiguis planis $v$. concaviusculis, margine circumalatis $v$. exalatis, dorso gibbo-convexis, vugoso-tuberculatis, medio in cuspidem brevem horiwontalem productis. Semen reni forme, plano-convexum, verticale, testa membranacea. Albumen farinaceum. Embryo periphericus cyclicus; cotyledonibus semiteretibus, radicula infera. - Herbae senegalenses et capenses, annuae, subsimplices sive ramosae, erectae vel diffusae; caulibus superne in cymas corymbiformes $\mathbf{v}$. semel aut pluries iteratas elongato-racemiformes solutis. Folia carnosula, linearia, alterna, exstipulata. Flores in racemis solitarii v. gemini, subsessiles, remoti.

OBS. Genus a cl. Gay in Bulletin sc. nat. Sept. 1829. Vol. XVIII. p. 412. constitutum, Limeo (Dicarpeae Presl, Gaudiniae Gay) proximum, carpidiorum marginibus plurimum circumalatis, loculorum spuriorum defectu, et situ stylorum faciei commissurati contrariis, nec parallelis insigniter diversum.

48. Semonvillea fenestrata Fenzl. S. glaberrima, raamosa ; ramis virgatis, in cymas axillares et terminales semel v. pluries iteratas, elongato-raceniformes solutis ; foliis linearibus carnosis planis et teretiusculis; floribus ejusdem cymae apetalis et corollatis, remotis, sepalis mucronulatis, petalis calycem subsuperantibus; carpidiis alatis cum exalatis varie alternantibus, alis orbicularibus basi apiceque retusis pellucidis, nervo rigido viridi peripherico cum aliis e disco concolori, obsoletius recte v. oblique cuspidato, radiatim emanantibus arcuatim anastomosante cinctis, facie symmetrice ideo fenestratis.

Patria. Cap. b. spei. Hab. prope Lekkeræing in Namaqua parva Hottentottorum, inter flumina Koussie et Garip comprehen sa $\left(28^{\prime \prime} 30^{\prime}-29^{\prime} 40^{\prime}\right.$ l. austr.) terrae inferioris occidentalis, all. 1500_2000' (Drège Plant. cap. n. 3157.) Flor. Sept. 
Herba verosimillime annua, erecta $v$. adscendenti-diffusa, glaberrima, \%viridi-glauca, ramosa, ramis ! crassitie pennae corvinae v. fili emporetici mediocris, simplicibus et asymetrice alternatim ramulosis, virgatis, semi-bipedalibus visis. Folia carnosa, linearia, obtusa, alterna, remota, erecto-patentia v. flaccile patula, nec amplexicaulia, nec decurrentia; inferiora $2^{\prime \prime}$ saepius longa, planiuscula, $1^{\prime \prime}$ ut plurimum lata, versus basim magis attenuata; juniora et superiora $1^{\prime \prime}$ potissimum longa ac $1 / 2{ }^{\prime \prime}$ crassa, teretiuscula. Cymae axillaribus saepe auctae, aphyllae, bracteolatae, semel, 2_4ter-iterato-dichotomae, cum perlunculo, longitudine vario, 2_6unciales, demuin racemiformes, remotiflorae. Bracteolae subulatae imoque setaceae, pedicellos calyce 3-plo breviores aequantes v: subsuperantes, membranaceae, herbaceo-carinatae. Calyx in alabastro oblongus, demum ovoideus v. semiglobosus, $1 \frac{1}{2}-21 / 2^{\prime \prime \prime}$ longus, sepalis ovalibus oblongisve mucronulatis, saepe inaequalibus, petaloideo-membranaceis, facie enerviis, medio herbaceis, carnosulis, profunde viridibus. Corolla saepius nulla v. 5-petala, alba, regularis, calycem potissimum triente superans, petalorum lamina subrotunda v. latissime ovato-subrotunda, integerrima, flabellato-venosa, in unguiculum anguste lineatem, glaberrimum, ipsa duplo breviorem rursus coarctata. Stamina 7 , aequilonga, calyce breviora, 5 sepalis alterna, 2 in aestivatione interioribus (sepalis) opposita, hypogyna; filamentis planiusculis, intus obsolete carinatis, e basi lata ovato-triangulari subulatis, inferius per fimbrias crispatas in cupulae speciem connexis. Antherae oblongo-lineares, loculis appositis parallelis subliberis, connectivo brevissimo lineari glandula eformi-incras sato croceo inhaerentes, versatiles. Ovarium lenticulari-compressum, bi loculare, biovulatum, abortu partiali subinde uniloculare uniovulatum, ovulis placentae basilari, dorso cuivis carpophyli adnatae, oblique adscendenti, apice in funiculum umbilicalem obsoletissimum liberum productae affixis, campylotropis. Styli duo filiformes, erecti, longitudinaliter intus stigmatosi, loculis oppositi. Fructus dicarpellaris, siccus, subcartilagi neo-crustaceus, calycem parum superans, carpidiis indehiscentibus, in toro obsoletissime conico parallele equitantibus, orbiformibus, faciebus commissuralibus (florum ejusdem racemi) nunc circumalatis planis, nunc exalatis concaviusculis, contiguis, demum solutis liberis, delabentibus, dorso gibboconvexis, ruguloso-tuberculatis, medio in cuspidem conicum brevissimum horizontalem rectum v. sursum deorsumve curvatum productis, basi emaiginata foraminulo, transitu contextus cellularis fecundationem facilitantis inserviente, instructa; ala marginem faciei commissuralis cujusvis carpidii cingens, discum latitudine adaequans, orbicularis, apice et basi retusa, pellucida, rigida, haud plicata, nervis 9 -13 viridibus radiantibus indivisis $v$. bifidis, cum peripherico concolori arcuatim anastomo santibus in fenestras subrotundas, obovatas, rotundato-trapezoideas $v$. triangulares elegantissimas divisa. Semen plano-convexum, reniforme, loculi cavitatem implens, verticale, testa membranacea testacea. Emhryo" periphericus cyclicus; cotyledonibus semiteretibus incumbentibus albidis, radicula infera.

49. Pittosporoun Fitgelianmme Putterlick. $\mathbf{P}$. foliis oblongo-lanceolatis apice acutis margine integerrimo revolutis, supra glabris laevibus nitidis, subtus pallidioribus fulvo-tomentosis, coriaceis, pedunculis in apice ramulorum binis ternisve, singulis e gemma prodeuntibus unifloris, floribus violaceo-purpureis, capsulis bivalvibus crassis polyspermis.

Habilat in Nova-Hollandia orientali extratropica. (Hiigel.) 
Fruticis uti videtur mediocris, summitates ramorum solummodo ante oculos habeo vix spithamam longas, teretes, pennae corvinae crassitie, apice in ramulos binos ternosve discedentes, cortice rubro-fusco, inferne glubriusculo, in junioribus pilis fulvis tomentoso vestitas. Folia alterna, superne congesta, petiolata: petiolo vix pollicis trientem longo, semitereti, in junioribus fulvo-tomentoso, erecto-patente; lamina horizontali, ad duos usque pollices longa, latitudine maxima sextam longitudinis partem vix superante, supra luteo-viridi. Pedunculi ad pollicem longi, crassiusculi, fulvo-tomentosi, ima basi bracteis 7_10 imbricatis stipati. Calycis foliola ovato-lanceolata, duas lineas longitudine superantia, extus pilis fulvis obsita. Corollae petala calycis foliolis alterna, oblongo-obovata, ad semipollicem long 8 , apice rotundata. Stamina calyce dimidio longiora, filamentis planis subulatis, antheris elongato-ovoideis luteis. Ovarium subsessile, ellipsoideum, fulvo-villosum, uniloculatum, multiovulatum. Stylus longitudine ovarii. Ovula marginibus placentarum in parietibus utrinque prominentium biseriatim affixa, horizontalia, anatropa. Capsula bivalvis, valvis crassis, coriaceis, intus purpureo-fuscis, verniceo-nitidis. Semina $7-9$, succo hyalino, spisso, glutinoso, amaroaromatico, resinoso in globum compacta, elongato-ovoidea $v$. reniformia, angulata, fusco purpurascentia, glaberrima, nitida.

OBSERVATIO. Species P. bicolori Hook. proxima, loco natali, florum situ, mag: nitudine et colore distincta.

50. Pittosporum densinorum Putterlick. P. foliis ellipticis cuspidatis, margine integerrimo subundulatis, tenue coriaceis opacis, utrinque in nervo mediano petioloque pilis brevibus strictis rufo-fuscis apice glanduliferis hirtis, pedunculis terminalibus ramosis in corymbum densiflorum dispositis cum pedicellis calycibusque glanduloso-hirtis, floribus fulvis.

In dumetis montium Indiae orientalis subtropicae florentem legit Hügel.

Arbusculae pius quam humanae altitudinis ramos video bipedales, teretes, pennae anserinae tenerioris crassitie, strictos, cortice pallide fusco, inferne cicatricibus hinc inde notato vestitos, superne foliosos, apiceque in ramulos 2_4, pilis brevibus, apice glanduliferis, rufo-fuscis hirtos discedentes. Folia alterna, petiolo circiter semipollicari, semitereti, erectopatente, in laninam explanata horizontalem, a longitudine pollicis ad quinque et dimidium usque extensam, in medietate tres octonas longitudinis latam, flavo-virentem. Pedunculi e gemma terminali prodeuntes, singuli basi bractea stipati. Calycis foliola ovata, vix lineam longa, apice acuta, subfusca, dorso hirta, marginibus pallidioribus ciliata. Corollae petala obovato-lanceolata; quadrilinearia, lineam lata, apice acuto, in alabastro subviolacea. Stamina tubo corollae quidquam longiora. Ovarium subsessile, ovoideum, fulvo-villosum, intus e semiseptis in axi fere con. tiguis incomplete biloculatum, loculis tri-quadriovulatis. Stylus ovario fere longior. Ovula in marginibus semiseptorum utrinque biseriata, horizontalia v. subadscendentia, anatropa. 
Nr. 6.

\section{NOVARUII STIRPIUII}

\section{DECADES}

\section{Editae a Mruseo Caesareo palatino Vindobonensi.}

Nr. 6. Vindobonae, typis Sollingerianis. 20. Junii 1839.

CROSSOPTERYX Fen:l-Endl. gen. plant. suppl. n. $\frac{3279}{1}$. Inflorescentia corymboso-paniculata, aphylla. Flores ...... Capsula cartilagineo-crustacea, calycis tubo adnato venulosorugulosa, ecostata, globulosa, faciebus latioribus sulco longitudinali laevi exarata, areola terminali, lata, calycis laciniis haud coronata, convexa, obsoletissime apiculata, nuda, bilocularis, loculicide dehiscens, valvulis medio septiferis, vix apice fissis. Placentae loculos omnino implentes, hemiellipsoideae, sarcomatosae, dissepimenti medio peltatim affixae, marginibus facieque interna liberis, demum subcartilagineo-induratae, solutae. Semina cujusvis loculi definita 7 , quinque placenlae foveolis dorsalibus, tumide marginatis, duo faciei comissurali semiimmersa, peltata, adscendentim imbricala, compressa, oblonga, testa reticulari-cellulosa, adpressissima, in alam mullipartito-laceram, laciniis sinubus rotundatis basi confluentibus, supra medium rigide setaceo-fimbriatis, undique marginantem oblongam, haud emarginatam producta. Embryo in axi albuminis parci, carnosi orthotropus; cotyledonibus planis ovalibus; radicula infera, brevi, tereliuscula, crassa. - Frutex Africae boreali-orientalis tropicae; ramulis foliisque utrinque hirtis, demum glabris, oppositis, late ovalibus rotundatis ellipticisque acuminatis, chartaceis, nec coriaceis, penninerviis, subtus laxe venulosis; stipulis liberis, adpressis, e basi lata ovata cuspidatis, pubescentibus, deciduis.

Genus inter Cinchonaceas genuinas primum ac hucusque unicum (exclusis Danaidis speciebus mauritianis) ex Africa tropica relatum, licet deficientibns proh dolor floribus, jam placentarum fabrica seminumque conformatione ac numero definito optime stabiliendum, prope Stevensiam collocandum, cui capsulae indole ac placentione inter contribules proximum, inflorescentia Hymenopogoni, Cosmibucnae et Calycophyllo prae reliquis accedells. 


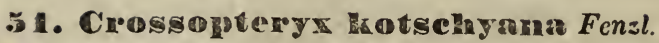 \\ 10}

In Africae boreali orientalis tropicae provincia Schangul legit cl. Kotschy (n. 532).

Frutex incertae altitudinis, cujus ramulos foliiferos, et inflorescentias sejunctas solum video, palmares ac pedales, crassitie pennae corvinae ad illam fili emporetici mediocris, obtuse quadrilateros, cortice fusco, superius cinerascente, pube horizontali brevissima, densa, hirta vestitos, decussatos. Folia cum petiolo teretiusculo tres ad sex lineas longa, bisexuncialia, opposita, basi articulata, demum decidua, minora plurimunı ovalia, obtusa, 13 lineas ad 2 pollices lata, majora elliptica, $2 \frac{1}{2}$ pollices haud superantia, potissimm angustiora, chartacea, nec coriacea, integerrima, penninervia, juniora supra hirta, infra laxe venulosa, pube concolori densiore, granulis atris sessilibus, copiosissimis, sub lente solum conspicuis permixta, subtomentosa, seniora supra laevissima, inferius ad costas pubescentia. Stipulae intrafoliaceae, inter se liberae, demum deciduae, inembranaceae, e basi lata, ovata in cuspidem rectum aequi-v. sesquilongiorem, subulatum, adpressum productae, pubescentes, fuscae, petiolo dinidio v. paruon ultra breviores. Flores non visi, in paniculis diametri $1 / 2-2-u n c i a l i s$, tri-tetrachotome ulterius divisis corymbose conferti, singulis paniculis ramulos florigeros simplicissimos, enodes, per paria internodiis, 2-, 11/2, 1-uncialibus depnumque brevioribus sejunctos, singuli paris aequilongos, rigide patentes terminantibus, per universales pąriter exactissime corymbiformes, diametro 3-7-unciales, digitales, palmares et spithameas, verosimillime in frutice axillares dispositis. Bracteolae deciduae ..... Pedicelli cum ramis ac ramulis inflorescentiae pube brevi horizontali densissima cinerea velati, demum $2-3^{\prime \prime \prime}$ longi, in speciminibus exsiccatis plurimum cernui visi. Capsula calycis tubo membranaceo, firmissime adnato, exsucco, transversim ruguloso, obsoletissime decemnervio, glabro vestita, globulosa, in ellipsoideum inferius vergens, 4-5 lineas Ionga, magnitudine fructus. Cerasi avium, e dúobus faciebus compressiuscula, simulque sulco longitudinali laevi utrinque-medio exarata, areola magna, ovali, nuda, convexa, in angulum obtusissimum, medio obsolete apiculatum, demumque transverse fissum compressiuscula coronata, cartilagineo-crustacea, bilocularis, sero in valvas hemiellipsoideas, apice laevissime fissas, ultra medium loculicidoseptifrage dehiscens; valvulis dissepimentum superius regulariter, infra medium, lamina placentifera vasornm fasciculo centrali indurato firmata incolume "rélicta, irregulariter rīptum medio sustinentibus. Placenta cujusvis locouli hemiellipsoidea, sarcomatosa, purpurea, totam cavitatem adimplens, facie convexa foveolis, tumide marginatis, tribus superioribus ac duabus inferioribus, facie plana dissepimenti completi, superius magis incrassati ac vi facile in laminas duas fissilis medio per papillam minimam peltatim affixa, simulque foveolis duabus instructa, demum subcartilagineo exsiccata, soluta. Semina definita 7 , placentae foveolis semiimmersa, peltata, adscendentim imbricata, oblonga, facie dorsali plana, ventrali convexinscula, medio elevato-nmbilicata, ferruginea, ala concolori, diametrum ejus breviorem haud superantem, pariter oblongam v. ellipticam hand emarginatam, in lacinias inaequales, sinubus rotundatis basi angustiore saepissime varie pertusa confluentes, supra medium et circa apicem latiorem elegantissime ac rigide setaceo-fimbriatas fissam cincta, cum ala $2-3$ lineas longa; testa reticulata, adpressissima, ejusque prolongationes inarginales ad lentem fibrilloso-cellulares. Embryo in axi albuminis carnosi parci orthotropus; cotyledonibus planis, ovalibus, subfoliaceis; ralicula brevi, teretiuscula, crassa, basim loculi spectante. 
oBSERVATIO. Fructus immaturi pressione fortiori multo facilius septicide quam loculicide in valvulas findendi, aqua vero bulliente tractati calyceque adhaerente orbati, iterumque exsiccati, propria vi elastica ad suturam dorsalem maturorum adinstar dissiliunt, quod ideo probe notandum, cum fere omnes in vertice areolae fissurani mi nimam dissepinenti laminas septicide separantem exhibueriut.

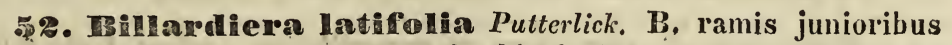
villosis, foliis inferioribus ovato-suborbicularibus, superioribus ovatis, integerrimis v. obsolete crenulatis villosulis, pedunculis calyce brevioribus calycibusque hirsulis, baccis ellipsoidea-cylindricis villosis.

\section{Ferd. Bauer Ic. $t .515$.}

\section{In Nova-Hollandia orientali prope Nelv-Castle legic Ferd.} Bauer 5. Aprili 1804.

Fruticis, ut videtur humilis, rami praestant sesquipollicares, teretes, pennae corvinae crassitiem vix superantes, superne sensim attenuati, stricti vel in inferiore parte hinc inde flexi, in sese circa axim torti, cortice juxta gyros longitudinaliter angulato-striato, rufo-fusco, glabro, nitido, in parte inferiore foliorum delapsorum cicatricibus toruloso, superne dense vilioso-incano vestiti. Ramuli teneriores alterni, intervallo circiter bipollicari dissiti, erecto-patentes, strictiusculi, villosi, suyerno tomentoso-incani. Folia solitaria, serie spirali ramornm ambitum cir. cumeunte alterna, internodiis dimidio pollice panllo brevioribus sejuncta, petiolo brevi, duas lineas vix metiente, semitereti, supra canalicnlato, infra convexo inserta, circumscriptione variantia, inferiora ovatosuborbiculata, superiora imprimis in ramis junioribus ovata, ad pollicem circiter longa, latitudine tres quartas partes longitudinis haud superantia, apice obtusa, basi abruptim in petiolum angustata, marginibus integerrimis vel hincinde obsolete crenulatis revoluta, patentia, flavo-virentia, subtus imprimis juniora in petiolo et nervo mediano villosula, adulta glabrata, in axillis gemmas vel fasciculos juniorum breves gerentia. Pedunculus duas circiter lineas longus, teres, gracilis, sub anthesi nutans, hirsutus. Calycis foliola peduncnlo linea longiora, e basi ovata subulata, subfusco-lutea, marginibus membranaceis pallidiora, extus in dorso convexiusculo marginibusque hirsuta, intus concava, glabra, patentia, apice reflexa. Corollae petala calyce plus duplo longiora, obivato-oblonga, unguibus angustioribus conniventia, laminis in maxima latitudine sesqui.

- lineam latis patentia, apice acuto recurva, citrina. Stamina ad duas tertias petalorum partes attingentia; filamentis complanato-subulatis, luteoalbidis. Antherae subgloboso-compressae, apice obtusae, baseos emarginatae dorso affixae, erectae, biloculatae, loculis appositis, dorsa connectivo adnatis, superne contiguis, inferne subdivergentibus. Ovarium ellipsoideo-cylindricum, fulvo-villosum, superne in stylum dimidio ipsius breviorem, stigmate dilatato, in centro elevato, in ambitu marginato, obsolete bilobo, papilloso coronatum staminaque vix superantem continue transiens. Ovula ovoidea, micropyle prope umbilicum extrorsum juxta funiculi insertionem aperta. Bicca cylindrico-ellipsoidea, utrinque obtusa, stylo persistente superata, densissine fulvo-villosa. Semina magnitudinem seminum Papaveris seminiferi vix superantia, badia, corrugatoangulata, sub testa tenera intus inembrana teneriore transparente obvestita, albumen albidum, carnoso-corneum, embryonis vestigium nondum offerens continentia. 
53. Hillarolierge gromelinora Putterlick. B. ramulis hispilulis, foliis oblongo-linearibus integerrimis, junioribus imprimis subtus sericeo-hirsutiusculis, pedunculis flore brevioribus calycibusque hirsutis, baccis cylindricis villoso-hirtis, floribus magnis aureis.

\section{In Nova-Hollandia orientali legit Ferd. Bauer.}

Media inter $B$. scandentem et angustifoliam, prima facie foliis ot floribus majoribus saturatioribusque distinguenda. Ramos volubiles video tripollicares, teretes, pennae corvinae crassitie, tortuosos, sat dure lignosos, cortice secundum gyros longitudinaliter rugoso-striato, fusco-badio, laevi, in parte inferiore lenticellis elevatis epidermidem rumpentibus, dilutioribus, foliorumque delapsorum cicatricibns exasperato vestitos. $R a-$ muli ex axillis foliorum aut jam delapsorum ant hinc inde superstitum juxta totam ramorum longitudinem alternatim prodeuntes, ad duos circiter pollices dissiti, bi-tripollicares, teneriores, patentes, juniores praecipne apicem versus dense hirsuti. Folia solitaria, serie ranulorum ambitum spiraliter circumcurrente alternatim dissita; infima in ramulis minima, bracteaeformia, propinquiora, patentia; intermedia sensim majora, longius distantia, patentissima; suprema maxima, conferta, erectopatentia; omnia basi angustiore semitereti nodulose incrassata petiolum mentiente subsessilia, lanceolato-linearia, longitudine paucarum linearum ad duos pollices cum dimidio longae, in latitudine maxima vix tres lineas superantia, plana, apice acuto, marginibus in siccis subrepando-revoluta, in pagina superiore saturate luteo-viridia, nonnisi ad nervum medium impressum pilis raris adpressis luteo-incanis obsita, subtus pallidiora, pilisque consimilibus ad nervum medium prominulum confertioribus sericeo-hirsutiuscula, adulta glabrata. Pedunculus circiter sesquipollicem longus, teres, gracilis, pendulus, e luteo canescenti-hirsutus, basi hinc inde folio uno alterove minimo bracteaeformi stipatus. Calycis foliola orato-subulata, acuta, longitudine pedunculi, subfusco-lutea, secus illis prioris speciei conformia. Corollae petala calyce plus duplo longiora, obovato-oblonga, in alabastro primum oviformi subconico apice acuto marginibus imbricatim sese tegentia, convoluta, sub anthesi in tubum calyce duplo longiorem, inferne unguibus angustioribus marginibus contiguis ad calycis usque longitudinem ampliorem, superne e laminis pro parte conniventibus marginibusque sese invicem amplectentibus angustiorem conniventia, suprema laminarum parte ad dnas lineas lata patentia, apice acutiusculo recurva, coloris ex sicco aurei, nervulis subfuscis praeprimis in ungue percursa. Stamina calyce vix longiora, filamentis subulatis, albidis. Antherae oblongae, obtusae, baseos emarginatae dorso affixae. Ovarium cylindrico-ellipsoideum, densissime fulvo-villosum, in stylum ipsius dimidio breviorem, stamina vix superantem, stigmate semigloboso obtuse bilobo terminatum desinens. Ovula ovoidea v. cordiformia. Fructus nonnisi immaturus in promptu, baccatus, densissime fulvo-villosus, in loculis semina plurima badia, corrugata occultans.

54. Dawiega alternifolia Endl.in Annal.Wien.Mus, II. 199. D. foliis alternis oblongis apice subspinescentibus, involucri subtriflori bracteis scarioso-membranaceis ovalibus planis.

Halital in Novae-Hollandiae auslro-occidentalis colonia KingGeorges-Sound. (Hügel.) 
Ramus pedalis, angulatus, striatus, glaber. Folia vera in ramulis squamaeformia, lineari-lanceolata, adpressa, dimidiam lineam longa, remote alterna, folia phyllodinea in ramis alterna, inferiora tres v. quatuor pollicis partes dissita, in superiore rami parte magis approximata, haseos torsione subverticalia, oblonga, duos pollices longa, tres $v$. tres et dimiảiam lineam lata, coriacea, basi angustata, ima basi utrinque denticulo setaceo, uncinatim recurvo aucta, nervo medio lateralibusque marginantibus utrinque prominulis, apice in spinam brevissimam coeuntibus, secundariis pinnatis, intra marginem reticulo venoso combinatis. Pedunculi in foliorum phyllodineorum axillis solitarii v. in apice rami oppositifolii, folio paullo breviores, compressi, angulati, obsolete puberuli, squamis coriaceis acutis, lineam longis, adpressis, remote alternis muniti, apice tri- v. interdum quadriflori. Pedicelli duas lineas longi, ebracteolati. Bracteae pedicellorum numero, scarioso-membranaceae, fuscescentes, glabrae, ovales, planae, pollice paullo breviores, uninerves, reticulato-venosae, apice brevissime mucronatae. Calyx campanulatus, hilabiatus, parce pilosus, labio superiore truncato, inferiore trifido. Corollae vexillum longe unguiculatum, trilineare, alae vexillo fere aequilongae, carina subfornicata, obtusa, hrevior. Stamina libera. Legumen coriaceum, glaberrimum, sexlineare, dorso recto, ventre convexo triangulare. Semen maturum unicum, umbilico strophiolato.

55. Daviesia ternata Endl. l. c. D. foliis ternatis oblongis apice subspinescentibus, involucri triflori bracteis scarioso-membranaceis obovatis planis.

Habitat in Novae-Hollandiae austro-occidentalis colonia KingGeorges-Sound. (Hügel.)

Species praecedenti valde similis, sed folia phyllodinea constanter ternata, bipollicaria, crassius coriacea et apice acutiora, pedunculi axillares solitarii, dimidiam folii longitudinem paullo superantes, squamis bilinearibus, acutis, patentiusculis, confertioribus vestiti, involucri bracteae obovatae, octo lineas longae, pedicelli calycesque magis pilosi.

56. Haviesia Dppositifolia Endl. l. c. D. foliis oppositis ternatisque ellipticis submucronatis marginatis involucri triflori bracteis coriaceis complicatis.

\section{Habitat in Novae-Hollandiae austro-occidentalis colonia King-} Georges-Sound. (Hügel.)

Rami angulato - tetragoni, glabri. Folia phyllodinea subdecnssatim opposita v. nonnulla ternata, paribus per internodia quinque linearum remotis, coriacea, elliptica, pollicem v. sesquipollicem longa, quatuor lineas lata, basi angustata ibique saepissime torta, uninervia, callosomarginata, mucrone brevissimo terminata, parce venosa, glaberrima. Pedunculi in foliorum axillis solitarii, eodem paullo breviores v. quidquam longiores, flexnosi v. strictiusculi, squamis paucis, acutis, adpressis, remote alternis muniti, apice ut praes:edentes species triflori. Bractcoc iuvolucri crassiusculae, coriaceae, $3 / 4$ pollicis longae, medio complisatar, 
Juae exteriores marginibus hinc valvatin approximatis, illine remotis, tertiam interiorem saepius planiusculam includentes, obsolete venosae, glaberrimae, extus rubiginoso-fuscescentes, intus albidae. Pedicelli biliseares, ebracteolati calycesque glaberrimi. Flores ut in speciebus pracedentibus.

OBSERVATIO. Daviesiae species $h$. $l$. descriptae, $\boldsymbol{h}$ congeneribus bractearum insolita maguitudine distinctissimae, affinite naturali proxime accedunt Daviesiae um. bellulatae $\mathrm{Sm}$.

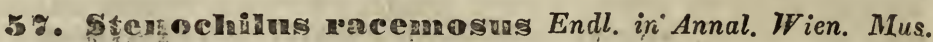
1I. 202. St. glaberrimus, foliis oblongo-lanceolatis basi angustatis integerrimis, pedunculis folia superantibus flexuosis in apice ramorum subracemosis.

In Novac-Hollandiae austro-occidentalis interioribus, itinere 100 mill. ab oriente urbis New-York, mense Decembri 1836 legic cl. Rö̈.

Ramuli pennae corvirae crassitie, teretes, glaberrimi, cortice rufo, nitido. Folia confertim alterna, oblongo-lanceolata, basi angustata, acutinscula, pollicem longa, duas lineas lata, integerrima, glaberrima, avenia. Flores in axillis supremorum foliorum, paullo reliquis breviorum solitarii, longiuscule pedunculati, racenum terminalem mentientes. Pedunculi ebracteolati, teretiusculi, superne subincrassati, foliis sextante v. quadrante longiores, flexuoso-declinati. Calyx herbaceus, ad hasim quinquepartitus, laxiusculus, laciniis ovato-acuminatis, duas lineas longis, sesquilinearn latis, margine obsoletissime membranaceis, omnibus acqualibus, basi imbricantibns. Corolla octo lineas longa, ex sicco aurantiaca, subclavato-tubulosa, tubo ima basi globose constricto, superne ampliato, limbo tubo paullo longiore, bilabiato. Labium superius rectun, dorso convexum, apice breviter quadrifidum, laciniis acutis, sesquilinearibus, duabus interinediis paullo altius connatis, Jateralibus margine vix reflexis, explanatum quatuor lineas latum, intus venosum, pilis raris albis conepersum. Labium inferius superiori aequilongum, lineare, acutum, sesquilineam latum, subdeflexum. Stamina 4 , didynama, supra corollae hasim globosam inserta, breviter exserta; filamenta e basi complanata paullulum dilatata filiformia, antherae reniformi-bilobae, loculis apice confluentibus. Ovarium ovatum, glaberrimum, calycis longitudine, quadriloculare, ioculis uniovulatis. Stylus filiformis, apice uncinato-incurvus, corollae longitudine; stigma minute capitatum.

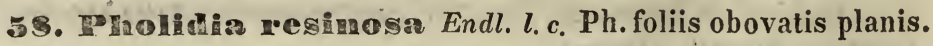

In Novae-Hollandiae austro-occidentalis interioribus, inter Swan-River et King-Georges-Sound, mense Decembri 1835 legit cl. Roë.

Ramuli fili emporetici crassitie, obsolete tetragoni, furfure stellato albido densissine obtecti, tuberculis resinosis subglobosis crebris, epidermidem lacerantibus conspersi. Folia approximatim alterna, obovata, quatuor lineas longa, sesquilineam vo duas lineas lata, plana, basi angustata, apice obtusa $v$. in acumen brevissimum patentiusculum contracta, crassiuscula, plana, glandulis immersis subrugosa, utrinque pilis 
stellatis canescentia. Pedunculi axillares solitarii, hrevissimi, vix dimidia linea longiores. Calyx profunde quinquefidus, basi angulatus, tres lineas longus, laciniis lineari-lanceolatis, acutis, squamulis furfuraceis minutissimis conspersis. Corolla infundibuliformis, sex et dimidiam lineam longa, tubo cylindrico, calycem vix superante, fauce ampliata, hinc gibba, apice subcontracta, limbo vix dimidiam lineam superante bilabiato, labio superiore bilobo, recurvo, inferiore trilobo aequali patulo, extus praeter imam tubi partem pilis stellatis furfuraceis conspersa, intus praeter lineam barbatam antice decurrentem glabra. Slamina tubo inserta, quatnor didynama, inclusa; filamenta basi barbata, antherae biloculares, loculis divaricatis, barbatis, apice confluentibus. Ovarium ovatoconicum, dense tomentosum, quadriloculare, loculis uniovulatis. Stylus filiformis, glaberrimus; stigma minute capitatum, vix emargiratum.

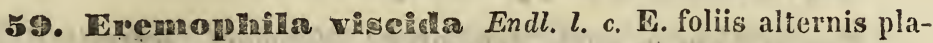
nis elliptico-lanceolatis integerrimis, pedunculis axillaribus solitariis v. congestis.

\section{Crescit in Novae-Hollandiae austro-occidenialis interioribus.} (Rö̈.)

Ramuli pedales, teretes, cortice rubro-fusco, viscido, nitente. Folia alterna, dimidium pollicem dissita, elliptico-lanceolata, sesquipollicem v. dnos pollices longa, quatuor v. quinque lineas lata, utrinque basi in petiolum brevissimum angustata, uninervia, obsolete venosa, utraque pagina nitida, viscida, marginibus integerrima. Pedunculi in axillis foliorum solitarii, gemini $v$. terni, uniflori, ebracteati, quatuor $\mathbf{v}$. quinque lineas longi, compressi, sursum dilatati, utrinque carinati, glabri. Calyx quadrilinearis, quinquepartitus, laciniae obovatae, obtusae, subaequales, fructiferae auctae, scariosae, reticulato-venosae, quinque lineas longae, quatuor lineas latae, duac interiores pallo angustiores. Corollae tubus amplas, quinque lineas longus, quatuor lineas latus, limbus trilinearis, bilabiatus, labia subaequilonga, superius fornicatum, breviter emarginatum, inferius trilobum, lobis obtusis, intus striis purpureis interruptis pictis. Siumina quatuor, didynama, paullo supra corollae basim inserta, longe exserta; filamenta filiformia, glaberrima, antherae reniformi-bilobae, loculis apice confluentibus, glaberrimis. Ovarium ovatum, compressiusculum, villosum. Ovula in loculis solitaria, pendula, anatropa, Stylus filiformis, glaberrimus; stigma minutissine capitatum, indivisum. Drupa subcompressa, bicarinata, pubescens, duas et dimidiam lineam longa, sesquilineam lata, quadrilocularis. Semina in loculis solitaria, inversa.

PLINTHUS Feñl. Calyx tubulosus semiquinquefidus, laciniis erectis, subinaequalibus, intus coloratis. Petala nulla. Stamina 5, imo calyci, nec ad sinus, libere inserta, laciniis alterna; filamentis exserlis. Antherarum loculi lineares, discreti, infra apicem coadunati. Ovarium triloculare, loculis 1-ovulatis, ovulis ex apice columellae centralis pendulis. Siylus 3-partilus, cruribus stigmatosis teretibus, erectis. Capsula calyce libero inclusa, oroidea, inferius membranacea, superius sublignosa, dense papillosa, 
apice rolundala, nec depressa, 3-locularis, loculicide 8-valvis, loculis monospermis. Semina pendula, pyriformia, nitida, seriatostrialo-sulcata. Embryo uncinatus; radicula supera. - Fruticulus capensis, habitu Kolleriarum quarundam, squarrosoramosus, humifusus, imbricato-foliatus, pube malpighiaceo-biacuminata coniplanata adpressa sericeus. Folia minima, ovato-triquetra, opposita et alterna, exstipulata. Flores 2-1-bracteolati, in axillis foliorum absconditi, sessiles, alterni.

Obs. Genus Galeniae proximum, staminibus solitariis subhypogyne insertis et capsulae conformatione diversum, Aizoideis, Portulacearum tribui secundae, adsociandun. ( $C f$. Annal. Wien. Mus. Vol. 2. inedit.)

\section{Plinthus eryptocarpus Fenzl.}

Patria. Cap. b. spei. Hab. prope Rietpoort, terretiorii Nieuvoveld dicti, plagae superioris occidentalis, alt. 3000_3500' (Drège pl. Cap. no. 8024?) Fl. Nov.

Suffrutex humilis, pube plana adpressa medifixa malpighiaceo-biacuminata sericea dense velatus, squarroso-ramosus, ima basi torulosus, polycephalus, ranis uncialibus et digitalibus, rigidis, crassitie fili emporetici tenuioris, diffusis, erectis et adscendentibus, tota longitudine ramulis simplicissimis, $2-5$ "' longis, oppositis alternisque. erecto-patentibus, subbiserialiter per internodia 1-6" longa remotis, arctissime imbricatofoliatis obsessis. Folia ad ramulorum innovationes disposita majora, sul-opposita et alterna, ovata, profunde canaliculato-triquetra, acuta, rigide patenti-recurva, fragilia, $2-3^{\prime \prime \prime}$ longa, basi $1^{\prime \prime \prime}$ lata ; ramulorum arctissime imbricata, obsolete 3 -5fariam disposita, ovato-triquetra, crassiuscula, dorso rotundata, acutiuscula, $3 / 4-11 / 2^{\prime \prime \prime}$ longa, in axillis modo flores, modo juniorum glomerulos depauperatos foventia. Flores $1 / 2-2 / 3^{\prime \prime \prime}$ longi, 1-2bracteolati, foliolis ramulorum absconditi, axillares, sessiles. Bracteolae foliis homomorphae, flores subaequantes. Calycis laciniae intus flavae, extus pube supra dicta dense velatae, anthesi patentes, serius clausae. Stamina 5, calycis tubo subhypogyne inserta, laciniisque alterna, parum exserta, capillaria. Semina minutie capsulae spectata magna, $1 / 3$ " diametro najori lata, pyriformia, compressiuscula, faciebus purpureofusca, dorso nigricantia, nitidula, seriato-sulcato-striata. Embryo radicula productiore uncinato-annularis. 
Nr. 7.

\section{NOVARUII STIRPIUI}

\section{DECADES}

\section{Editae a Mruseo Caesareo palatino Vindobonengí.}

Nr. 7. Vindobonae, typis Sollingerianis. 30. Juni 1839.

FRIEDRICHSTHALIA Fenzl. - Endl. Gen. plant. Suppl. n. $\frac{3779}{1}$. Calyx quinquefidus, serius particus, demum vesiculosoincrescens, amplissimus, laciniarum maximarum faciebus latéralibus valvatim replicatis prismatico-quinquealatus: Corolla hypogyna, hypocraterimorpha, fauce pervia gibbis quinque, laciniis alternis(!), planiusculis angustata, tubo amplissimo brevi, inferius tereti, superius faciebus intrusis pentagono, laciniis mucronato-cuspidatis, in alabastro per aestivationem plicativam contortis, margine undulatis. Antherae quinque, medio tubo adnatae, sessiles, sagittato-subulatae, longe acuminatae, in conum conniventes, acuminibus spiraliter contortae, dorso villoso-tomentosae. Ovarium quadrilobum. Stylus subulatus. Stigma simplex, punctiforme. Nux unica, tribus reliquis (semper?) hebetatis, naxima, orbicularis, tomentosa, margine papilloso-denticulato inflexo, umbilico ventrali latissimo, concavo, styli basi suborbiculari oblique incumbens. Semen orbiculare, concavo-convexum, testa membranacea viridi, venosa, villo arachnoideo tenul velata. Embryo exalbuminosus, cotyledonibus foliaceis, radicula minima, tereti, supera. - Herba Africae boreali-orientalis tropicae, perennis, foliis alternis et oppositis sessilibus, striguloso-leprosis, floribus speciosis albidis, fauce flavis, gibbis purpureis (?) pictis, longe pedicellatis, demum pendulis, racemoso-paniculatis.

Genus inter Borrágineas tribus Cynoglossearum Trichodesmati proxime affine, characteribus carpicis Omphalodi et Cynoglosso aliquo modo accedens.

Dixi in honorem amicissimi Emanuelis de Friedrichsthal, botanices cultoris indefessi, larga stirpium rariorum messi e Graecia et Macedonia feliciter relata, Americae aequinoctialis plagas nunc explorantis. 


\section{G1. Friedrichsthalia physaloides Fenzl.}

\section{In Africae boreali-orientalis tropicae provincia Schangub} regni Faะঞokel mense Januario legit Kotschy (n. 577.)

Herba perennis, glauca, erecta, 1-2pedalis visa, caulibus e rhizomate crasso sublignescente, ut videtur, tortuoso enascentibus, strictis, angulatis, ima basi hispidis, reliqua parte laevissinis, pruinosis, plurimum crassitie calami scriptorii, medulla spongiosa farctis, simplicissimis, in racemos validos, elongatos, alternos, per paniculam patula in dispositos demum solutis. Folia sessilia, semiamplexicanlia, inferiora saepius opposita, nec connata, nec decurrentia, reliqua alterna, per internodia seminncialia et 2 - 4 pollices longa varie remota, ramormm non effoetorum foliola pauca, ab aliquibus lineis ad unciam dimidian longa, in axillis plerumque foventia, infima et suprema minora, lanceolata, $1-1 \frac{1}{2}$ uncialia, media majora, ovato-lanceolata, acuta, plurimum 11/2- $2 \frac{1}{2}$ pollices longa, fere cuncta basi subcordata, erecto-patula, subchartacea, supra uni-, subtus penninersia, utrinque (densiusque plurimum superne) tuberculis callosis, planis, albidis, inermibus sive seta brevissima, farcta, patentiuscula terminatis, orbicularibus, punctiformibus, subinde (imprimis subtus) varie confluentibus, plurimis consita, facic quasi lepidotoleprosa, margine integerrimo tuberculis similibus scabra. Racemi digitales et spithamei, simplicissimi $v$. inferius uno alterove minore aucti, asymetrice alterni, folio bracteali basi haud suffulti, hinc e simplici axeos florigeri centralis divisione, nec gemmarum axillarium in ramos explicatione orti, 3-16fori, foliolis linearibus ac lineari-subulatis, 2_5 lineas longis, sparsis obsessi, paniculam amplam pyramidatam vo ovoideam efformantes Pedicelli basi plurimum ebracteati, fliformes, apice leprosi, turbinati, caeteruin laevissimi, post anthesim ulterius increscentes, demum 1_2 pollices longi, subsecundi, cernui. Flores speciosi. Calyx in alabastro ovoideus prismaticus, acute pentagonus, ad trientem 5 fidus, lemum laciniarum marginibus, in angulosiv. potius alas valvation replicatis, mediantibus tuberculis setigeris leviter coalitis, secedentibus usque ad basim 5-partitus, anthesi 5-8 lineas Jongus, ulterius increscens demuin 1 - $1 \frac{1}{2}$ uncialis, in morem Nicandrae physaloidis late quinquealatus, vesiculoso inflatus, laciniis ovatis, sinubus reflexis quasi latissime cordatis, acuninatis, conniventibus, facie uninerviis, glabris, basi dense le. prosis, reliqua parte plus minusve punctato-tubercnlatis, primum rore glauco suffusis, demum flavescentibus. Corolla hypocrateriformis, calycem parum superans, tubo amplo lacinias orbiçlares, in alabastro per aestivationem plicativam acute pentagono-pyramidate convolutas, anthesi horizontaliter expansas, undulatas, medio in cuspidem mucroniformem tortam productas, albidas, in sicco flavas aequante, fauce pervia, gibbis 5 , intus leviter protrisis, oblongis, apice rotundatis, medio sulco longitudinali percursis, glabris, purpureis (?), in medium tubum deorsum prodnctis, laeiniis alternis, parum angustata, flava. Antherae quinque, medio tubo ad gibborum basim insertảe, sessiles, sagittato-subulatae, longe acuminatae, in conum longe exsertum, postice lanuginoso- albo tomentosum, apice glaberrimum conniventes, acuminibus spiraliter contortis, loculis appositis, longitudinaliter intus dehiscentilus. Ovarium quadrilobum, 4 ovulatum, ovulis duorum loculorum contiguorum parieti communi infra apicen lateraliter affixis, pendulis, anatropis, micropyle supera. Stylus inter ovarii lobos transiens subulatus, primum erectus, demum marcescens, flaccide ac varie curvatus, stigmate terminali simplicissimo, punctiformi. Nux unica, reliquis (florum 18 examinatorum) omnilius, post anthesim statim hebetatis, vesicnlarum varie compressarum, inaequalium, forma persistentibns, chartacea, ovali-subrotunda, semiuncialis, dense incano-tomentosa, con- 
vexiuscule depressa, marginibus inflexis papilloso-denticulatá, facie ventrali latissima, concava, nitida, styli basi planiusculae ovali-subrotundae oblique incumbens. Semen nuci conforme, concavo-convexum, testa viridi, venulosa. tomento arachnoidec tenero fatiscente obducta. Embryo exalbumınosus; cotyledonibus maximis foliaceis orbicularibus, radicula supera tereti, minima.

Nuces Trichodesmatis zeylanicae et africanae crustaceas, racie dorsali convexa glaberrimas nitidas, ventrali styli baseos quadrangurars foveis profundis inmersas in anguium compressas ruguioso-iacunose exsculptas, vidco.

HALGANIA Gaudichaud ad Freyc. 448. Endlicher Gen. plant. Suppl. $n . \frac{3749}{1}$. Calyx campanulatus, quinquefidus. Corolla hypogynä, rotata, quinquepartita. Stamina 5 , imae corollae inserta, ejusdem laciniis alterna; filamenta brevissima, libera, antherae introrsae, biloculares, ovato-oblongae, basi emarginata insertae, in acumen membranaceum lineare productae, in conum quinquepartibilem cohaerentes, loculis apice rima brevi dehiscentibus. Ovarium sessile, biloculare, loculis indivisis $v$. semisepto ex axi producto incomplete bilocellatis. Ovula in loculis gemina, collateralia, in medio dissepimento semianatropa, micropyle supera. Stylus filiformis, simplex; stigma capitatum, minimum. Capsula coriacea, subcylindrica, bilocularis, bipartibilis, loculo altero minore efoeto, fertili monospermo. Semen pendulum. Embryo ..... - Frutices Novae-Hollandiae austro-occidentalis; foliis sparsis, ellipticis v. linearibus integerrimis, v. cuneatis dentatis apice trilobis, estipulatis, corymbis terminalibas v. lateralibus ebracteatis. floribus caeruleis.

62. Iralgania anagalloides Endl. in Annal.Wien. Mus. II. 204. H. pilosa, foliis sparsis cuneatis utrinque unidentatis v. integerrimis apice trilobis, lobis obtusis muticis vo mucronatis, corymbis lateralibus simplicibus.

In Nova-Hollandia austro-occidentali, inter Swan-River el King-Georges-Sound legit cl. Roë.

Species Halganiae littorali Gaudich. op. cit. t.59. quantum ex icone judicare licet, valde affinis, sed omnium partium minutie, corymbis lateralibus, et fortassis etiam fructu satis distincta. Ramulum video quadripollicarem, lignosum, ramulis plurimis, strictiusculis, teretibus, cortice fusco-rugoso, pilis rigidis adpressis cinerascente vestito auctum. Folia sessiliz, sparsa, in ramulis junioribus conferta, cuneata, crassiuscula, sesquilineam longa, apice tres quartas partes lineae latitudine sua haud superantia, basi angustata, medio utrinque, imprimis inferiora, denticulo brevi obtuso aucta, v. saepins integerrima, apice triloba, lobis brevibus, obtusis, muticis $v$. in mucronem brevissimum, rigidum, candicantem, deciduum desinentibus, substantia crassiuscula, facie subcanaliculata, utrinque pilis rigidis, raris, adpressis, sub lente candicantibus cinerascentia. Corymbi laterales, simplices, tri-quadriflori, semipollicem v. pollicem longi, pedunculo stricto, pedicellisque filiformibus ebracteatis, pilis adpressis cinerascentibus, floriferis strictis, fructiferis apice nutantibus. 
Calyx tubo anguste campanulato-cylindrico, dimidiam lineam Iongo, et limbi quinquepartiti laciniis angustissime lineari-lanceolatis, acutis, adpresse cinereo-pilosis, fructiferi tubas parnmper anpliatus. Corolla hypogyna, decidua, rotato quinquepartita, tubo brevissimo, lineae quadrantem vix aequante, limbi pulcherrime caerulei lobis obovato-oblongis, obtusis, sesquilineam longis, dimidiam latis, aestivatione contortim imbricatis, sub anthesi patentibus. Stamina 5, imo corollae tubo inserta, ejusdem lobis alterna; filamenta brevissima, vix tubo corollae longiora, subulato_complanata, antherae introrsae, biloculares, ovato-oblongae, basi emarginata insertae, apice in acumen membranaceum lineare planum productae, in conum duas lineas et qnadrantem longum, apice tubulosum, quinquedentatum, stylo pervium, quinquepartibilem cohaerentes; loculi appositi, lineam longi, flavicantes, subcartilaginei, superne caernlescentes, utrinque imprimis dorso pubescentes, intus prope apicem rimula brevi, poriformi, demum elongata aperti; acumina antherarum unam lineam et quadrantem longitudine aequantia, glaberrima, nitide caerulea. Discus v. glandulae nullae. Ovarium liberum, ovatum, minimum, biloculare. Ovula in loculis gemina, collateralia, medio dissepimento inserta, utrinque cavitatis longitudine aequaliter producta, parte superiore apice micropyle majuscula aperta, inferiore hinc rhaphe procurrente notata, chalaza punctiformi terminata. Stylus filiformis, glaberrimus, caerulescens, extra antherarum tubum ad quadrantis lineje longitudinem prominens; stigma nudo oculo haud conspiciendum, sub lente minutissime capitatum, glaberrimum Capsula coriaceo-subcartilaginea, sesquilineam longa, inaequilatere subcylindrica, teres, ima basi incrassata, ad tertiam longitndinis partem calycis tubo immersa, ejusdem laciniis patentissimis cincta, fusca, apice obtusa, areola minima ex insertione styli notata, abortu unilocularis, monosperma, loculo altero penitus obliterato v. multo minore, a fertili separabili. Semen loculum implens, subcylindricum; testa tenuissime membranacea, chalaza nigricante basim loculi respiciente, extremitate supera attenuata, subtruncata. Albumen (?) carnosum.

63. Halgania integerrima Endl. l. c. H. glabriuscula, foliis lineari-ellipticis integerrimis, corymbis lateralibus simplicibus.

In Novae-Hollandiae austro-occidentalis interioribus, legit cl. Rö̈.

Ramuli quam in praecedente specie teneriores, glabriusculi, pilis rarissimis adpressis conspersi. Folia sparsa, intervallis unius lineae sejuncta, lineari-elliptica, $2 \frac{1}{2} 2^{\prime \prime \prime}$ longa, $1 / 2^{\prime \prime \prime}$ lata, utrinque angustata, crassiuscula, parcissime adpresse pilosa, apice obtuso subcallosa, nervo uno facie impresso, dorso obsoleto. Corymbi laterales semipollicem longi, bi-quadriflori, pedunculo pedicellis filiformibus breviore. Flores plane ut in specie praecedenti, partibus omnibus vix quidquam majoribus. 
64. HaIgania lavandalacea Endl. $\boldsymbol{l}$. c. H. dense tomentosa, foliis ellipticis subtus trinerviis integerrimis, nervis lateralibus marginantibus, corymbis subterminalibus simplicibus depauperatis.

In Novae-Hollandiae austro-occidentalis interioribus, inter King-Georges-Sound et Swan-River legit cl. Rö̈.

Fruticulus ut videtur humilis, statura tamen multo quam specierum supra descriptarum validiore, deusissime cano-tomentosus. Ramum video lignosum, semipedalem, subtetragonum, superne teretem, penna corvina paullo crassiorem, tomento denso cinerascente tectum. Folia sparsa, internodiis trium v. quatuor linearum sejuncta, sessilia, elliptica, utrinque obtusa, dimidium pollicem longa, sesquilineam v. duas lineas lata, supra plana, subtus nervis tribus valde prominentibus, medio duobusque lateralibus marginantibus, folii marginem revolutum mentientibus notata. Ramuli axillares, semipollicares pollicaresque, strictiusculi, foliis multo minoribus, $2 \frac{1}{2}{ }^{\prime \prime \prime}$ longis, $1 / 2$ latis, confertioribus, albo-tomentosis, ceterum aeque trinerviis vestiti. Corymbi in apice ramulorum bi-triflori, v. abortu uniflori, pednnculo brevi pedicellisque $3^{\prime \prime \prime}$ circiter longis, ut et calyce pube glandulosa capitata ferrugineo-nigricantibus. Calyx $2 \frac{1}{2}$ lin. longus, tubo subcampanulato, brevi, limbi quinquepartiti laciniis linearilanceolatis, acutis. Corolla ut in praecedentibus speciebus, multo major, limbi laciniis tres lineas longitudine superantibus. Antherarum tubus tres lineas longus.

DIPLOCHONIUM Fen:l. Calyx ultra medium 5-filus, tubo infundibuliformi, laciniis petaloideo-membranaceis coloralis apice fornicato-mucronatis, facie sinulus acute prominentibus deflexis coronato. Petala nulla. Stamina definite plurima (40_60), summae calycis fauci linea circulari tumidula aequabiliter nec per phalanges inserta, plura tamen inter lacinias congesta, inaequalia. Antherae didymae, ellipsoideae, versatiles. Ovarium liferum, calycis tubo semiimmersum, biloculare, loculis $\infty$ ovulatis, vvulis amphitropis, tola longitudine columellae centrali funiculis distinctis affixis. Styli 2, elongati, filiformes, tota facie interna stigmatosi, basi subhori\%ontales, ad angulum fere rectum infracti, crure altero longiore adscendente. Capsula ellipsoideo-oblonga, bilocularis, ad calycis tubi faucem transversim dehiscens, operculo obtuse conico chartaceo, segmenti inferioris epicarpio ab endocarpio, aeque tenerrime membranaceo, marginibus introflexis axim placentariam sustinente, perfectissime soluto, persistent:. Semina subreniformia, nitidula, laevia, atra, hilo extremilati radiculari proximo, lato, transersso, foraminiformi instructa. Embryo uncinatus, fere cyclicus. - Herba capensis, succulenta, papulosa, dichotome ramosa. Folia opposita, carnosa, integerrima, plana, obovata et elliptica, marginibus revolutis imoque faciebus retrorsunı conduplicatis. Flores in ramorum dichotomiis sessiles alares, supremi axillares. 
Genus Portulacearum ordinis, Sesuvio proximum, ovario biloculari et singulari fabrica segmenti inferioris capsulae, (unde etiam nomen: infundibuli duplicali) nec non seminibus hilo foraminiformi lato insignitis, diversum. (Cf. Anna?. Wiener Mus. V. II. inedit.)

\section{Diplochonium sesuwioides Fenzl.}

Patria. Cap. b. spei. Hab. in rupestribus ad Gaprium fluvium lateris coloniae occidentalis alt. 500' (Drège pl. cap. no. 2938!)

Radix et inferiores stirpis partes mihi ignotae, ramo florigero semipalmari tantum viso. Herba habitu Portulacae oleraceae v. Sesuvii Portulacastri, verosimillime prostrata, dichotome squarroso-ramosa, suc:culenta, glabra, papulis sive granulis alhidis undique adspersa, ramis teretiusculis, crassitic pennae corvinae. Folia opposita, carnosa, plana, facic enervia, obovata, elliptica et spathulata, margine revolnta, faciebus subinde retrorsum conduplicata, hinc acutiuscula, hinc obtusa, in petiolum basi membranaceo-dilatatum attenuata, patentissima, infima uncialia, superiora sensim breviora. Flores in ramorum dichotomiis solitarii, remoti, supremi confertiores et dichotomia ramulorum imperfecta axillares redditi, sessiles, magnitudine Sesuvii Portulacastri, in sicco e roseo in flavum vergentes visi. Calyx infundibuliformis, patentissimus, semiuncialis et parum longior, limbo ad duas trientes 5 -fido, laciniis ovatis petaloideo-scariosis, medio herbaceis, apice cucculato in mucronem carnosum filiformem recurviusculum $1^{\prime \prime \prime}$ longum productis, tubo obconico, sinubus laciniarum in angulos acutos prominentes deflexos protrusis summo margine pentagono. Petala nulla. Stamina numero varia 40 - 70 , linea circulari tumidula extimae calycis faci aequabiliter inserta, pluribus tamen, licet non per phalanges distinctis, saltem circa sinus confertioribus. Filamenta setacea inaequilonga, calycis laciniis triente breviora, aurea. Antherae ellipsoideae, versatiles. Ovarium calycis tubo semiimmersum 2-loculare, loculis $\infty$-ovulatis. Styli 2 , staminibus longiores, filiformes, tota longitudine intus stigmatosi, lbasi ad angulum fere rectum infracti, crure breviore horizontali. Capsula ellipsoidea 2locularis, medio circumscisse dehiscens, axi placentario persistente, operculo obtuse conico subchartaceo, segmento inferiore calycis tubo immerso membranaceo, separatione spontanea epicarpii ab endocarpio, in dissepimenta introflexo, in infundibula dua semet excipientia imaque basi solum subcohaerentia secedente. Semina plurima, subreniformia, $1 / 2^{\prime \prime \prime}$ fere lata, laevia, nitidula, atra, hilo amplo transverso foraminiformi. Testa crustacea. Embryo radicula longius producta magis uncinatus quam cyclicus, albumini parco circumpositus.

66. Conospermenn Hitigelii $R$. Brown msc. 1836. C. ifoliis lineari-filiformibus subcanaliculatis obtusiusculis pedunculisque elongatis scapiformibus, spica terminali densa, rhachi bracteisque serice-pubescentibus, perigonii glaberrimi limbo tubo multo breviore.

\section{Crescit in vicinia coloniae Swan-River. (Hïgel.)}

Radix pennae anserinac crassitie, simplex, parum tortuosa. Caulis abbreviatus, vix pollicaris. Folia congesta, ima basi dilatata imbricantia, lasinscule patentia, lineari-filiformia, tres ad quatuor pollices longa, sub- 
compressa, laevia, glaberrima, obsolete canaliculata, apice obtusiuscula. Pedunculi e summis foliorum alis solitarii, prima facie terminales, sesquipedales bipedalesque, simplicissimi, stricti, compressiusculi, striati, glaberrini. Spica terminalis, simplicissina, densa, ante anthesim ovata, pollicaris, demum elongata. Rhachis pube sericea, demum evanescente. Bracteae menbranaceae, late ovatae, cuspidatae, duas lineas longae, basi totidem lineas latae, basi sericeae, margine baseos puberulae, apice coloratae. Perigonium glabrum, tubo tres lineas longo, fauce constricta, limbi sesquilinearis labio superiore latiore, fornicato, inferioris lobis tribus in unicum, apice trifidum cohaerentibus. Stamina basi laciniarum inserta, posticum antherae loculis duobus perfectis, lateralium altero multo minore, antici ambobus minimis, efoetis. Ovarium dense hirtum, uniloculare, ovulo unico, erécto. Stylus perigonii longitudine, e basi tenuissime capillari sursum crassior, flexuosus, medio subincrassatus; stigma obtusum, suboblique terminale. Nux obconica, depressiuscula, apice densissime sericeo-papposa.

6\%. Comospermum Ianiforum Endl, in Annal. Wiener. Mus. II. 208. C. foliis oblongo-lanceolatis acutis trinerviis tenuissime pubescentibus basi tortis verticalibus, pedunculis axillaribus elongatis ramosis, spicis alternis perigoniisque subglobosis densissime albo-lanatis.

\section{In interioribus Novae-Hollandiae occidentalis, ab oriente} urbis Neu-York, mense Decembri 1836 legit cl. Rö̈.

hamulum habeo pedalem, strictum, pennae corvinae crassitio, cortice rubro, striato, pube sericea, rara vestito. Folia alterna, $8-4$ lin. dissita, oblongo-lanceolata, sesquipollice parum breviora, duas et dimidiam lineam lata, crassiuscula, coriacea, basi in petiolnm brevissimum, marginatum angustata, ibique torta, inde vertiralia, strictiuscula, apice acuta, in mucronem brevem, çollosum, nigricanteı, deciduum desinentia, utrinque tenuissime sericco-pubescentia, trinervia, nervis utraque pagina prominulis, medio validiore, lateralibus margini approximatis, nervis secundariis alternis acutangulis, inter nervos reticulato-venosis. Pedunculi e summis foliorum alis stricti, tri-quadripollicares, apice alternatim ramosi, ramulique spicas breves, densas, paucifloras gerentes, dense lanato-tomentosi. Bracteae sub ramulis e lata basi cuspidatae, sesquilineares, flore breviores, omnes apice glabratae. Perigonium subglobosum, diametro bilineari, extus densissime albolanatun, siccitate intus atropurpureum, tubo antice inflato, limbo tubi longitudine, bilabiato, labio superiore latiore, fornicato, obtusiusculo, inferioris tripartiti laciniis lana cohaerentibus, acutinsculis. Stamina basi laciniarum inserta, postici anthera biloculari perfecta, lateralium dimidiatis, loculo altero minimo efoeto, anticum anantherum, bifidum, laciniis acutis, sinu lato disjunctis. Ovarium obconicum, uniovulatum, apice pilorum corona vestitum, pilis complanatis rigidulis. Stylus basi capillaris, superne aequaliter incrassatus, inflexus; stigma prope apicem laterale, stamini sterili haud cohaerens. 
68. Conospermum Stoechadis Endl. . c. C. foliis semitereti-filiformibus apice callosis, pedunculis axillaribus elongatis apice corymbosis, spiculis alternis confertis perigoniisque dense lanatis. Hügel.

In vicinia coloniae King-Georges-Sound legic Caralus L. B.

Folia alterna, dimidium pollicem dissita, pedem longa, semiteretifiliformia, glaberrima, laxiuscule erecto-patentia, callo pyramidali acuto terminata, dorso convexo inultistriata, facie plana obsolete bisulea, basi ima subdilatata. Pedunculi axillares, foliis multo lungiores, compressi striati, inferne tenuissime tomentosi, snperne corymbosi, densissime albo-lanati. Spiculae alternae, confertae, primum subcylindricae, tandem paullo elongatae, densiflorae, pollicem v. sesquipollicem longae. Bractea e basi lata, ovata, lanata in cuspidem acutum, glabratum repente angustata. Perigonium duas lineas longum, limbo dimidia linea vix longiore, extus lana densa alba obsitum, intus in planta sicca atropurpureum, tubo subcylindrico, labio superiore obtusiusculo, inferioris tripartiti laciniis acutis. Stamina congenerum, antheris diutine in synantherium cohaerentibus, lateralium loculo altero plane obsoleto, sterile anticum hifidum, laciniis filiformibus. Ovarium obconicum, basi pilosum, cetera glabrum, apice truncato pilorum corona dimidiam styli longitudinem aequante cinctnm. Stylus e basi capillari flexuosa superne incrassatus, medio inflatus, staminibus nunquam cohaerens.

69. Marianthus exubeseens Putterlick. M. foliis ellipticis integerrimis, corymbis axillaribus subtrifloris folio brevioribus, calycis foliolis obtusiusculis ciliatis, petalis purpurascentibus.

\section{In Novae-Hollandiae austro-occidentaliz Colonia Swan-River} legit cl. Rö̈.

Fructicis ramos video pedales, teretes, penna corvina paullo crassiores, sursum sensin tenuiores, lignosos, dextrorsum volubiles et in sese circa axim tortos, cortice juxta gyros longitudinaliter rugoso-striato, rufo, e cuticula soluta hinc inde cinerascente, glaberrimo vestitos. $R a-$ muli in axillis foliorum plerumque bini, e gemmis superpositis prodeuntes, teneriores, patentiusculi, basi squamulis 5 _ 7 imbricatim sese tegentibus et persistentibus stipati, magnitudine inaequales; superiore utplurimum in longum crescente ramisque conformi ; inferiore florifero brevissimo, vix ultra lineam metiente, superne foliis duobus alternantibus in ambitu triente distantibus, uno inferiore breviore altero superiore longiore instructo, cortice pallide virente pube rara albida vestito. Folia solitaria, in ambitu ramorum alternatim dissita, petiolata, e petiolo patente semitereti supra canaliculato, infra convexo, basi nonnihil incrassata semilunari inserto, "3_5" longo, in laminam fere horizontalem, ellipticam, circiter duas pollices longam, semipollicem maxime latam, subcoriaceam continua, margine integerrimo revoluta, apice obtuso brevissime apiculata, medio nervo in utraque facie elevato, apicem versus sensim attenuato, nervisque lateraibus fere oppositis ex angulo sursum acuto prodeuntibus, inter se parallelis, interpositis venulis creberrimis reticulatim inter se anastoinosantibus, praecipue marginem versus arcuatim connexis percursa, juniora in petiolo subtusque pube rara longiuscula albida conspersa, adulta 
glaberrima, pallide fíavo-virentia. Pedunculus solitarius, teres, ad pollicem longus, filiformis, ochroleucus vel rubicundus, albido pubeseens, superne trichotomus. Pedicelli alternatim sibi invicem superpositi, teretes, vix quatuor lineas longi, filiformes, albido pubescentes, singuli basi bractea squamaeformi, obtusiuscula, lineam permetiente, panllo superius bracteolis minimis duabus, tribus vel quinque, modo confertis modo remotioribus, albido-pubescentibus caducissimis stipati, apice paullulum in tornm solidum, diametri linearis, centro elevatum ampliati, uniflori, inaequales, infimo longissimo primum florente, supremo minimo. Calycis hypogyni foliola quinque, cum bracteolis alternantia, ima basi in annulum cum toro margine connatum coalita, aestivatione imbricata, oblongo-obovata, lineae unius et dimidiae longitudinem aequantia, semilineam lata, acutiuscula, glabra, ex flavo purpurea, margine pallidiore ciliata. Corollae petala 5 , hypogyna, calycis foliolis alterna, aestivatione marginibus imbricatim sese tegentia, spathulata, ad pollicem usque longa, unguibns angustioribns, laminas triplo plus longitudine superantibus, erectis, in tubum conniventia, laminis in latitudine maxima ultra duas lineas permetientibus, sub anthesi patentia, apice acuto reflexa, extus in sicco purpurascentia, in unguilus intus pallidiora. Stamina 5, hypogyna, cum petalis alterna isque breviora, tubum vix superantia, inter se subaequalia, filamentis e basi plana latiore purpura diluta tincta superne filiformibus, candidis, erectis, post anthesim extrorsum flexis. Antherae terminales, ovatae, obtusae, lineam longae, baseos emarginatae dorso filamentis affixae, biloculares, loculis longitudinaliter adpositis, superne contiguis, inferne subdivergentibus, in connectivo adnatis, flavicantibus, introrsum juxta totam longitudinem dehiscentibus. Pollinis globuli subtriedri, lnteoli. Ovarium supra torum stipite brevi crasso basi glandulifero elevatum, elongato ellipsoideum, utrinque compressiusculum, glaberrimum, snperne in stylum teretem filiformem, ovario ter quaterve longiorem continue transiens, stigmate simplici truncato, fovoolis duabus papillosis instructo terminatum; intus septo longitudinali e semiseptis in axi contiguis formato dimidiatnm, biloculare, loculis multiovulatis. Ovula in marginibus carinae duplicatae, juxta axim dissepimenti in loculos prominentis utrinque biseriata, funicnlo brevissimo extrorsum curvato affixa, horizontalia, subglobosa; micropyle ad latus quoque externum prope nmbilicum aperta. Fructus immaturus hucdum visus, e receptaculo floris superstite stipite brevi suffultus, capsularis, stylo persistente superatus. Capsula elongato-ellipsoidea, utrinque compressinscula, parietibus juxta sulcum longitudinalem utrinque torulosa, marginibus obtuse angulatis sutura in tota longitudine decurrente duplicatis, secus glaberrima, membranacea, subfusca, intus septo longitudinali cum sulcis parietalibus continuo bilocularis, loculis polyspermis. Semina 12-18 in quovis loculo, plura abortiva, in marginibus carinae duplicatae, juxta axim dissepimenti funiculo brevişimo biseriatim suspensa, subglobosa, vix diuidiam lineam in diametro metientia, in facie semen collatere spectante quid quam gibla, rufa, glaberrina, laevia. Integumentum seminum e tenui cellularum strato formatum, rufo fuscum, intus membrana teneriore undique adhacrente, in regione chalazae internae placentulan cellularem vasis biramosis pertextam includente vestita, interius sacculum nembranaceum tenerrinum transparentem corrugatum incurvum, in una parte cum placentula, in altera cum micropyles puncto interno cohaerentem, in cavo secus inani includit.

\%. Mariantinus Moribundus Putterlick. M. foliis !lateellipticis utrinque acuminatis integerrimis, corymbis terminalibus breve pedunculatis multifloris, calycis foliolis acuminatis glaberrimis, petalis flavis. 
In Novae-Hollandiae austro-occidentalis colonia ad Sinum Regis Georgii legil Carolus L. B. Hügel.

Species elegans, cujus ramulum video unicum floriferum, sesquipede paullo longiorem, teretem, pennae corvinae crassitudine, dextrorsuı volubilem, cortice subrubello, glabro, laevi, nitido vestitum, intus sat dure et tenaciter lignosum. Folia solitaria, serie spirali alternatim in anlitu locata, interstitis circiter triente circumferentiae in latum et ultra tres pollices in altum dissita, superiora sensim propinquiora, omnia petiolata, e petiolo vix trilineari, semitereti, infra convexo, supra canaliculato, basi nonnihil incrassata semilunari inserto, genmulamque in avilla fovente glaberrimo, erecto-patente, in laninam explanata fere horizontalem, late ellipticam, hinc in petiolum, illine in cuspidem productam, tygs pollices longam, latitudine in inferioribus tres quartas partes, in supremis vix trienteı longitudinis attingentem, marginibus integerrimis subtilissime revolntam, subcoriaceam, flavo virentem, subtus pallidiorem, utrinque glaberrinam, laevem. Nervus petiolo continuus unicus validior medianus, in pagina superiore impressus, infra prominulus, apicem versu sensim attenuatus, fuscatus, emissis e latere utrinque nervis subtilioribus fere pinnatin decurrentibus, nervulosque reticulatim inter se anastomosantes exerentibus atque margine versus arcuatim confluentibus, mox iteram ex arcu nervulos proxime intra margineın in arcus congredientes emittentibus, tune ad apicem percurrens in apiculim brevissinum laminamque superantem desinens. Pedunculus in apice ramuli terminalis solitarius, vix pollicem longus, teres, crassiusculus, glaberrimus, superne alternation ramosus. Pedicelli e ramulis alternatim prodeuntes ternati, semipollice paullo longiores, teretes, strictiusculi, undique patentes uniflori, longitudine inaequales, infimi et extimi longiores, superiores et interiores sensim breviores corymbum constituentes, singuli basi bractea lanceolato-subulata, linea paullo longiore, glaberrima stipati, superne nonnihil in torum centro elevatum soliduı, partes florales gerentem incrassati. Flores hermaphroditi in alabastro oviformes, apice conico acnto, supremo centrali rachin continuante primo florente, mox in ramulo proximo inter ternos medio e. s. p. Calycis foliola quinque, hypogyna, lanceolato-subulata, ad quatuor circiter lineas longa, apice acuto patentia, margine integerrima, glalora, e flavo-purpurascentia. Corollae petala quinque, hypogyna, calycis foliolis alterna, aestivatione marginibus inbricatim sese tegentia, subspathulata, ad decem circiter lineas longa, unguibus dimidium fere longitudinis consumentibns et vix quadrantem lineae latis in tubum calyce dimidio longiorem conniventia, laminis planis in latitudine maxima tres lineas superantibus sub anthesi patentia, apice acuto reflexa, coloris in sicco saturate vitellini. Stamina quinque, hypogyna, petalis alterna, calycis foliolis Iongitudine aequalia, sub anthesi ovario adpressa, serius patentia. Filamenta subulata inaequaliter quadriquetra, aciebus prominulis sub lente minutissime serratis, candida, apice antherifera. Antherae oviformes, obtusae, baseos emarginatae dorso medio affixae, erectae, luteae, biloculatae, loculis appositis, introrsum longitudinaliter dehiscentibus. Pollinis globula triedra, luteola. Ovarium in centro tori subsessile, compresso-cylindricum, glaberrimum, superne in stylum conico-cylindricum, brevem, filiformem, stigmate subcapitato obsolete bilobo terminatum, staminaque vix superantem continuo transiens, intus biloculatum, loculis pluriovulatis. Ovula in dissepimenti angulo centrali juxta longitudinem biseriatim affixa, bina fere collateralia, horizontalia, anatropa; micropyle proxime, umbilicum septum spectans; rhaphe juxta faciem internam alteri ovulo obversam obsoleta, subito in chalazam paullulum elevatam [transiens. 


\title{
NOVARUII STIRPIUII
}

\section{DECADES}

\section{Editae a Murseo Caesareo palatino vindobonensi.}

\author{
Nr. 8. Vindobonae, typis Sollingerianis. 10. Julii 1839.
}

CESATIA Endlicher Gen. plant. n. 4357. Calycis tubus compressus, subdidymus, limbus brevissime quinquedentatus. Petala ovalo-lanceolata, acula, integra, apice recto. Fructus a latere plano-compressus, lasi subcordatus, apice emarginatus, mericalpiis suberosis, evitlatis, quinquejugis, jugo dorsali intermediisque obsoletis, lateralibus marginalibus productis, commissura bisulca. Semen sectione transversali ellipticum. - Herba NovaeHollandiae austro-occidentalis; foliis superioribus tripartitis, lobis linearibus, acutis, umbellis lateralibus terminalibusque simplicibus, quinquefloris, pedicellis involucri pentaphylli foliola lineari-lanceolata, integra paullo superantibus.

Genus dicatum honoribus Vincentii L.B. Cesati, Mediolanensis, qui nuper de Ambrosinia docte disseruit, et Umbelliferarum ordinem difficillimum egregiis studiis illustrat.

\%1. Cesatian orinata Endlicher in Annal. Wiener Mus. II. 200.

In Novae-Hollandiae austro-occidentalis interioribus legit cl. Rö̈.

Herbae, nt videtur humilis, nonnisi summitates quadripollicares prae occulis habeo, unde nec de statura nec de facie plantae, nec de foliis inferioribusquidquam tradere licet. Caulis summitates teretes, glabrae. Folia alterna, tripartita, lobi lineari-lanceolati, acuti, quatuor v. quinque lineas longi, dimidiam lineam lati, sub lente parce pilosi, petiolo quatuor lineas longo, basi dilatato. Umbellae simplices, laterales termi. nalesque, quinqueftorae, pedunculo pollicari. Involucri pentaphylli foliola lineari-lanceolata, sesquilineam longa, quadrantem lineae lata, acutissima, margine obsoletissime pilosa. Pedicelli involucri foliolis oppositi, floriferi iisdem paullo breviores, fructiferi sextante longiores, patentiusculi, teretiusculi, glabri. Calycis tubus compressus, subdidymus, viridis, glaber, jugis lateralibus marginalibus manifestis, contiguis, limbus brevissime quinquedentatus. Petala ovato-lanceolata, acuta, subcari- 
nata, integra, apice recta, carina violacea, marginibus membranaceis, albidis. Stamina petalis paullo breviora, flava. Styli 2 , filiformes, basi vix incrassati, divaricato-patentes. Fructus a latere plano-compressus, duas lineas longus, totidem lineas latus, basi subcordatus, apicis emarginati lobis arcte contiguis, stylos erectos velantibus. Mericarpia saepissime inaequalia, alterius dorso nempe versus apicem sensim deliquescente, tenuissime suberosa, sordide violacea, quinquejuga, jugo dorsali intermediisque duobus obsoletis, fructu maturo nonnisi epicarpio ibi obscurius colorato manifestis, lateralibus marginalibus in alas suberosas, angustas, crassiusculas, obtusas productis, alis inaequalibus, altera dimidio latiore fere dimidiam lineam aequante, mericarpii oppositi alam angustiorem amplectente, altera angustiore a mericarpii oppositi ala latiore amplexa. Commissura angusta, medio linea longitudinali convexa notata, ob juga marginalia eidem contigua bisulca, Carpophorum setaceum, lineam longum, indivisum, persistens. Semen sectione transversali ellipticump s angulo interiore acutiore.

MALLOPHORA Endlicher Gen. plant. Suppl. $\frac{3694}{1}$. Calyx quadripartitus, persistens, laciniis linearibus. Corolla hypogyna, infundibuliformi-tubulosa, tubo recto, aequali, calycem superante, limbi quadrifidi laciniis aequalibus, oblusis, erectiusculis. Stamina 4, corollae fauci inserta, breviter exserta, aequalia. Ovarium quadriloculare, loculis uniovulatis. Stylus filiformi-subulatus, exsertus; stigma obtusum. Drupa sicca, globosa, fragilis, quadrilocularis, axi hiante. Semina in loculis solitaria, erecta. - Frutices Novae-Hollandiae austro-occidentalis, dense lanatotomentosi; foliis oppositis, subsessilibus, rigidis, capitulis terminalibus solitariis v. corymbosis, floribus bracteatis, bracteis exterioribus involucrantibus, corollis albis.

2. Mrallophora globiflora Endl. in Annal. Wien. Mus. II. 206. M. capitulis solitariis.

\section{In Novae-Hollandiae austro-occidentalis interioribus, legit} cl. Roë.

Rami teretes, pennae corvinae crassitie, ramulique alterni et oppositi, subfastigiati, apice dichotomi, tomento stellari adpresso, sordide ferrugineo obtecti. Folia opposita, internodiis inter paria trium circiter linearum, juniora in vetustorum axillis juxta ramulos brevissimos sae. pe subfasciculata, elliptica, utrinque obtusa, duas et dimidiam lineam longa, unam lineam lata, petiolo brevissimo subsessilia, supra pallide viridia, tomento cinereo raro conspersa, marginibus revolutis rugosa, subtus tomento densissimo cinerea, nervo medio prominulo obtecto. Pedunculi in ramulis terminales, solitarii, semipollicares, tomento pallide ferrugineo conspersi, monocephali, Pedicelli sesquilineares, seni-quideni subcorymbosi, in capitulum approximati, lana densa, candidissima, patente vestiti, singuli bractea lineari, densissime albo-lanata stipati, extimorum quatuor v. quinque bracteis oblongo-lanceolatis, obtusis, sesquilinearibus, ferrugineo-tomentosis, involucrum constituentibus. Calycis quadripartiti laciniae exacte lineares, obtusae, lineam longae, intus virides, extus lana densa candida, in pedicellum decurrente vestitae, in glo- 
bum diametri bilinearis conniventes. Corolla hypogyna, tubo recto, aequali, intus sparsim puberulo, calycem paullo superante, limbi quadridifidi laciniae aequales, obtusae, erectiusculae. Stamina 4, corollae paullo infra faucem inserta, aequalia, breviter exserta; filamenta filiformia, glaberrima, antherae ovatae, utrinque obtusae, biloculares. Ovarium subglobosum, pilosum, quadriloculare. Ovula in loculis solitaria, adscendentia. Stylus terminalis, subulatus, basi pilosus, exsertus; stigma obtusum. Drupa parva, globosa, tomentosa, styli delapsi cicatricula umbilicata, calyce connivente recondita, fragilima, quadrilocularis, dissepimento ad $_{i}$ axim fiónte. Sesthena in loculis solitaria, erècta.

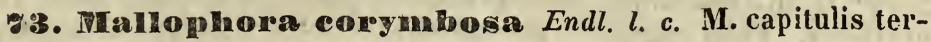
minalibus corymbosis.

\section{Crescic in Novae-Hollandiae austro-occidentalis interiori- bus (Roë).}

Differt a praecedente specie: ramis ramulisque densissime lanatotomentosis; foliis oppositis, quatuor lineas longis, unam lineam latis, utrinque densissime lanatis, marginibus revolutis rugosissimis, glabratis, pedunculi terminalis, pollicaris lana longa, fuscescente, patente, capiculis corymbosis, paucifloris, involucri bracteis calycibusque lana laxiore vestitis, floribus paullo majoribus.

CONOMIT RA Fenzl. - Endl. Gen. plant. Suppl. n. $\frac{3447}{1}$ Calyx quinquepartitus. Corolla campanulata, profundissime quinquepartita, tubo brevissimo tereti-cylindrico, laciniis linearibus calyce longioribus, utrinque glabcrrimis, fauce coronata forniculis subulato-filiformibus, brevissimis, sinubus insertis, basi triangulari dilatata in angulum intus prominula, decurrentibus. Corona staminea nulla. Columna staminea brevissima, corollae tubo parum exserta. Antherae appendice membranacea, triangulari-subulata brevissima terminatae. Pollinia compressa, reniformia, apice attenuato affixa, pendula. Stigma cylindrico-conicum, calyce longe exsertum, apice obtusum, integerrimum. Folliculi solitarii, erec$t i$, tenue cylindrici, versus apicem attenuati, longissimi, glaberrimi, striati. Semina plurima, oblonga, convexo-concava, ad umbilicum coma longissima coronata. - Herba Africue borealiorientalis tropicae, annua, erecta, ramosa, glabriuscula; foliis oppositis linearibus, acutis, uninerviis, aveniis, margine revolutis, herbaceis; Roribus interpetiolaribus, solitariis v. binis, brevissime pedicellatis, minimis, viridi-flavescentibus.

obServatio. Genus Parapodio E. Meyer proximum, corollae appendiculis corniculiformibus subulatis, nec squamaeformibus rotundatis, stigmate tereti-conico, nec pyramidali quinquesulcato, foliis membra. naceis aveniis, nec costatis, ac fortassis fructu (Parapodii pro tempore ignoto) diversum. 


\section{Comonkitra limearis Fenzl.}

\section{Habitat in. Africae boreali-orientalis provincia Cordofan, unde retulit cl. Theodorus Kotschy (n. 35.)}

Herba annua, erecta, glabriuscula, digitalis ac spithamea visa. Radix simpliciuscula, crassitie fili emporetici mediocris, testacea. Caulis ejusdem crassitiei strictus, basi tantummodo $\mathrm{v}$. ad mediam fere altitudinem ramosus, reliqua parte simplicissinus, a ramo ad ramum parum flexuosus, in sicco striatus, pallide virens, ad lentem pube minutissima densius leviusve consitus. Rami duo infimi oppositi, reliqui alterni, internodiis 3-6"' lg. discreti, simplicissini, erecto-patentes, fastigiati, canle senper breviores. Folia opposita, per iriternodia 3-10"' longa, sejuncta, primum erecta, demum flaccide patentia, brevissime petiolata, plana, membranacea, carnosula, linearia, utrinque attenuata, acutiuscula, margine parum revoluta, unum ad duos et dimidium pollicem longa, semilineam ad duas lineas lata, subtus glabra, uninervia, avenia, supra pube minutissima rara adspersa, v. omnino nuda. Flores solitarii $\mathbf{v}$, duo tresve in umbellulas interpetiolares sessiles conferti, uno tantum persistente fructifero. Pedicelli sub anthesi calycem aequantes, tenuissime filiformes, fructiferi incrassati, teretes, erecto-incurviusculi, sesquilineam longi, glaberrimi. Calyx viridi-flavescens, corollae concolor, lineam v. parum ultra longus, usque ad basinn fere quinquepartitus, subcampanulatus, laciniis ovato-lanceolatis, acutiusculis v. obtusis, apice conniventibus, glabris, enerviis. Corolla hypogyna, campanulata, profundissime quinquepartita, tubo brevissimo, laciniis linearibus obtusis, supra medium tortis, calycem duplo superantibus, flaccide patulis, utrinque glaberrimis, fauce coronata appendiculis quinque corniculiformibus, incurviusculis, setaceis, albis, sinubus insertis, basi triangulari in angulum intus prominulis, utrinque decurrentibus, foveolas corollae laciniis subjectas efformantibus. Corona staminea nulla. Stamina corollae tubo supra basim inserta, in tubum brevissimum pentagonum connata. Antherae erectae, introrsae, biloculares, loculis adnatis, parallelis, connectivo inmediatin supra loculos in laminam membranaceam, antherarmm fere longitudine, late triangularem, acuminatam producto. Pollinia reniformia, compressiuscula, pendula, extremitate angustiore retinaculis brevissimis, teretiusculis, clavatis, cum antheris alternantibus, stigmatis basi adhaerentibus, geminatim affixa. Ovarium sessile, conicum, e duobus carpidiis follicularibus, plurimum inaequalibus, arcte adpressis compositum, placentis ad suturam ventralem decurrentibus, multiovulatis. Stigma cylindrico-conicun, calyce longe exsertum, corollae lacinias saepe subaequans, esulcatum, apice rotundatum, integerrimum, demum spiraliter contortum, marcescens. Fructus folliculares, solitarii, stricte erecti, ad folliculum unicum, altero omnino abortivo, restricti, duos ad tres et dimidium pollicem longi, subcylindrici, sesquilineam lati, versus apicem subemarginatum attenuati, multistriati, glaberrimi, membranacei, albi, ad suturam ventralem longitudinaliter dehiscentes. Placenta tenuissime menbranacea, solubilis, fatiscens. Semina plurima, imbricata, pendula, oblongn linearia, extremitate inferiore parum latiore rotundata, superiore hilari angustiore coma pilorum simplicissimorum longissima nivea coronata, dorso convexa, facie ventrali concava, raphe decurrente subcarinata, marginibus integerrimis, subincurvis, membranaceo-extenuata, utrinque transversim rugulosa, grisea, $3^{\prime \prime \prime}$ et parum ultra saepe longa, lineam fere lata. Embryo in axi albuminis carnosi orthotropus; cotyledonibus planis, radicula brevi supera. 
9. Werticordib compta Endl. in Annal. Wien. Mus. II.194. V. lobis calycinis multifidis, laciniis lineari-subulatis plumoso-multipartitis, petalis staminibusque sterilibus fimbriatis, stylo incluso imberbi, foliis obovato-triquetris a latere compressis dorso laevibus, pedunculis subcorymbosis, bracteolis distinctis muticis.

In Novae-Hollandiae austro-occidentalis interioribus, ad orientem urbis New-York legit cl. Rö̈.

Affinis Verticordiae Hügelii, a qua foliorum forma, qua magis ad $V$. insignem accedit, facillime distinguitur. Ramuli stricti, teretiusculi, graciles, glabri, cortice cinerascente, rugoso vestiti. Folia opposita, garibus in inferiore ramorum parte internodiis trium linearum sejunctis, triquetra, a lateribus compressissima, duas et dimidiam lineam longa, dorso acute convexa, laevia, marginibus plana, unam lineam lata, facie angustissima, hasi excavata inserta, apice obtusiusculo mucronem brevissimum, oculo nudo vix conspiciendum gerentia, punctis glandulosis nigris, immersis notata, glaberrima. Pedunculi e summorum foliorum axillis filiformes, glaberrimi, quatuor lineas longi, corymbum terminalem formantes. Bracteolae membranaceae, concavae, pedunculi apici nonnihil incrassato opposite insertae, in alabastro marginibus arctissime applicitis cohaerentes, apice obtusissimae, demum patentes et mox deciduae, basi brevissima, subcupuliformi persistente. Calycis tubus obconicus, angulatus, basi patentim pilosus, superne glaber, limbi quinquepartiti lobi palmatin multifidi, laciniae basi purpurascentes, apice albae, plumoso-multipartitae. Petala 5, ovata, sesquilinean longa, fimbriata, uninervia, nervo saepius in aristam excurrente. Stamina basi brevissime monadelpha, fertilia decem, tereti filiformia, petala aequantia, cum totidem sterilibus, subpetaloideis, paullo longioribus, fimbriato laceris alternantia. Stylus staminibus brevior, imberbis; stigma capitatum.

\% G. Verticordia Toęi Endl. l.c. V. Iobis calycinis trifidis, laciniis tripartitis, lobulis lineari-subulatis plumoso-multipartitis, petalis fimbriatis, staminibus sterilibus indivisis, stylo subexserto imberbi, foliis imbricatis triquetris, pedunculis corymbosis, bracteolis distinctis muticis.

\section{In Novae-Hollandiae austro-occidentalis interioribus legit} cl. Roë.

Fruticulus semipedalis, divaricato-ramosissimus, glaber. Folia conferta, acute triquetra, lineam longa, dimidiam lineam lata, punćtata. Pedunculi glaberrimi, pollicares. Calycis tubus obconicus. 
\%. Verticordia picta Endl. l. c. V. lobis calycinis multifidis, laciniis lineari-subulatis plumoso-multipartitis, petalis ; integerrimis, staminibus sterilibus indivisis v. trifidis nudis, stylo incluso obsolete barbato, 'foliis subsemiteretibus mucronatis, pedunculis subcorymbosis, bracteolis distinctis dorso infra apicem mucronatis.

\section{In Novae-Hollandiae austro-occidentatis interioribus le-} git cl. Rö̈.

Fruticulus gracilis, ramosus, ramis filiformibus, cinereis, glaliris. Folia conferta, semitereti-filiformia, inferiora tres et dimidiam lineam longa, summa dimidio breviora, dorso convexo punctig glandulosis subaspera, facie plana, in mucronem brevem, uncinato-recurvum desinentia. Pedunculi e summorum foliorum axillis subcorymbosi, filiformes, glaberrimi, folio dimidio longiores. Alabastra subglobosa, bracteolis arcte cohaerentibus inclusa. Bracteolue membranaceae, concavae, dorso infra apicem mucronem brevissimum exserentes, purpurascentes, glaberrimae, glanduloso-punctatae, sub anthesi valvatim dehiscentes, mox ipsa basi solutae, deciduae. Calycis tubus late turbinatus, ima basi cum ovario connatus, supra ovariuin productus, limbi quinquefidi lobi membranacei, palmatim multipartiti, laciniae lineari-subulatae, circiter tres lineas longae, pulmoso-multipartitae, albae. Petala 5, late obovata, ungue brevissimo laminae carnosae calycis tubum intus vestienti inserta, pallide rosea, maculis purpureis picta. Stamina cum petalis inserta, in tubum membranaceum, vix usque ad mediam sui longitudinem vigintifidum connata, laciniae planae, e basi lata subulatae, alternae antheras oblongas, biloculares, dorso affixas gerentes, alternae anantherae, paullo angustiores, integrae $v$, apice trifidae, lacinulis acutis. Ovarium imo calycis tubo adnatum, vertice complanato disco epigyno, in laminam calycis tubum vestieritem continuato, tectum, uniloculare. Ovula 10, laminae discoideae, e basi loculi excentrice peltatae margini inserta, anatropa. Stylus staminibus brevior, subclavatus, infra stigma terminale capitatum obsilete larbatus.

9. Verticondia chrysantha Endl. l. c. V. lobis calycinis multifidis, laciniis lineari-subulatis plumoso-multipartitis, petalis fimbriato-multifidis, staminibus sterilibus cuneatis apice trifidis nudis, stylo incluso imberbi, foliis teretibus mucronatis, pedunculis subcorymbosis, bracteolis distinctis muticis persistentibus.

\section{In Novae-Hollandiae austro-occidentalis interioribus legit} cl. Rö̈.

Fruticulus divaricato-ramosus, ramulis tenellis, teretiusculis, subfastigiatis, cinereis, glabris. Folia opposita, in inferiore ramulorum parte per paria remota, duas et dimidiam lineam longitudine vix superantia, patentiuscula, versus eorundem apices conferta, adpressa, quinque lineas longa, omnia filiformi- v. subclavato-teretia, in mucronem brevissimum cartilagineum, subrecurvom desinentia, punctis glandulosis, immersis consita, glaberrima. Pedunculi in summorum foliorum axillis solitarii, corymbos parvos formantes, folio vix dimidio longiores, obsolete angu- 
lati, glaberrimi, virides. Bracteolae 2 , membranaceae, late concavae, muticae, pedunculi summo a pici basi subcallosa insertae, primum cohaerentes, sub anthesi patentes, altera exterior interiorem basi marginibus amplectens, delapso calycis limbo adhuc persistentes. Calycis tubus obconicus, brevis, supra ovarium brevissime productus, nitidus, glaber, limbus quinquepartitus, lobi palmato-multipartiti, lutei, laciniis subulato-linearibus, in lacinulas plurimas pinnato-partitis. Petala 5 , laminae carnosae calycis tubum intus vestienti inserta, ovata, lata basi sessilia, calycis limbo concolora et paullo breviora, subcartilaginea, in lacinias filiformes profunde fimbriato-partita. Stamina cum petalis inserta, decem sterilia, petaloitlea, cuneata, apice trifida, lobis deltoideis, acutis, lateralibus multo minoribus, petalis triente breviora, lutea; totidem fertilia, sterilibus alterna, ima basi iisdem cohaerentia, filiformi-subulata, inter se a equalia, sterilibus vix longiora, inflexa, antherae introrsae, biloculares, connectivum totum dorsum occupans, apice in glandulam sub aqua pellucidam tumens, loculi antice adnati, oppositi, basi turgidiores, longitudinaliter dehiscentes. Ovarium inferum, uniloculare. Ovula cavitatis basi carnosae excentrice inserta, gemina v. interdum terna, anatropa. Stylus staminibus brevior, imberbis. Sligma minute capitatum.

OBSERVATIO. Verticordia nitida (Clrysorrhoë nitida Lindl. in Bot.Mag. Com. pan. II. 357.) a nostra differt: ramalis atro-viridibus, pedunculis longioribus, petalis serratis, staminibus sterilibus subulatis, apice glandulosis.

9.7. Werticoredia grandifiora Endl. . c. V. lobis calycinis multifidis, laciniis lineari-subulatis plumose-multipartitis, petalis fimbriato-multifidis, staminibus sterilibus complanato-subulatis trifidis nudis, stylo incluso imberbi, foliis lineari-triquetris mucronatis, summis distiche imbricatis, pedunculis corymbosis, bracteolis distinctis muticis persistentibus.

In Novae-Hollandiae austro-occidentalis interioribus, centum milliarium itinere ad aquilonem ab urbe New-York legic cl. Roë.

Ramuli teretes, cortice cinereo, sub lente setulis minimis exasperato, phyllopodiis valde prominentibus toroso. Folia inferiora opposita, paribus internodio trium linearum sejunctis, in axillis juniorum fasciculos breves gerentia, ramulorum suprema distiche imbricata, omnia lineari-triquetra, inferiora quatuor lineas longa, summa duas et dimidiam lineam longitudine vix superantia, dorso convexo marginibusque acuta, laevia, glaberrima, facie plana, apice in mucronem brevissimum, rectum desinentia, punctis nigris, immersis conspersa. Pedunculi e summorum foliorum axillis solitarii, corymbum formantes, semipollicares, e basi filiformi sursum incrassati, subcompressi, glaberrimi, fuscescentes. Bracteolae 2, coriaceae, late ovatae, duas lineas longae, tres lineas latae, obtusissimae, concavae, dorso muticae, glabrae, fuscae, pedunculi apici basi callosa insertae, primum cohaerentes, sub anthesi ad mediam circiter longitudinem patentes, inferne altera exterior interiorem arcto amplexu retinens, ita ut prima fronte connatae videantur', sed revera distinctae, persistentes. Calycis tubus turbinatus, basi ad longitudinem dimidiae lineae supra ovarium productus; limbus quinquelobus, luteus, lobi tres lineas longitudine aequantes, paullo angustiores, in lacinias plurimas, lineari-subulatas, plumoso-multipartitas palmatifissae, patentes, laciniis pulcherrime luteis, apice interdum virescentibus. Petala laminae carnosae, calycis tubum intus vestienti inserta, ovata, calycis 
limbo paullo brevióra, basi fuscescentia, cartilaginea, in lacinias plurinias lutescentes, apice interdum virescentes palmatifissa. Staminu cum petalis inserta, iisdem alterna, sterilia decem complanato-subulata, acuminata, trifida, laciniis acuminatis, lateralibus paullo brevioribus, angustioribus, divaricatis, fertilia filiformi-subulata; antherae biloculares, locnli lutei, connectivo dorsali crassiusculo, fusco antice alnati, basi turgidiores. Ovarium inferum, disco epigyno, in laminam staminiferam, calycis tubum intus vestientem producto, obtectum, uniloculare. Ovula paca, e basi erecta, anatropa. Siylus staminibus brevior, imberbis. Stigma terminale, minute capitatum, glabrum.

80. Anthocereis anisantlas Endl. A. viscoso-pubescens, ramis ramulisque spinescentibus, foliis in ramulorum axillis fasciculatis oblongo-cuneatis obtusis integerrimis, corollae laciniis tribus posticis brevioribus.

\section{In Novae-Hollandiae austro-occidentalis interioribus,}

cum Halgania anagalloide legit cl. Roë.

Summitates video semipedales, lignosas, pube viscosa, glauco-viridi consitas, patentim ramosas, ramis ramulisque in spinas transformatis. Ramuli teretiusculi, bi-quadripollicares, subflexuosi, spinis alternis, quatuor $\mathbf{v}$. quinque linearuon intervallo remotis, patentiusculis, quatnor v. quinque, lineas longis, in mucronem rigidum desinentibus. Foliorum ramos v. spinas fulcientium nonnisi paucissima supersunt, plurima in ramorum, pauca in spinarum axillis fasciculata, oblongo-cnneata, tres lineas longa, unam lineam lata, apice rotundata, versus basim angustata, integerrima, substantia crassiuscula, subtus uninervia, dense viscosopubescentia. Pedunculi e foliorum fasciculis subsolitarii, filiformes, circiter tres lineas longi, viscoso-pubescentes, et ut videtur ebracteolati. Calycis tubus subcampanulatus, una linea paullo brevior, quinquenervis, nervi virescentes, glanduloso-pubescentes, in limbi quinquepartiti lacinias excurrentes, interstitia et limbi sinus submembranacei, laciniae linearilanceolotae, acutae, lineam longae, duae posticae paullo longiores. Corolla infundibuliformi-campanulata, ut videtur flava, tubus duas lineas longus, ima basi subglobose coarctatus, superne ampliatus, intus glaberrimus, striatus; limbi quinquepartiti laciniae anguste lineares, acutae, patentes, duae anticae duas lineas longae, tres posticae sesquilinea haud longiores, paullo reliquis angustiores, duae laterales inter se aequales, intermedia aliquantulum brevior. Stamina corollae tubo supra basim ejusdem globoso-contractam inserta, quatuor didynama, fancem aequantia, duo postica paullo longiora. Fulamenta e basi nonnihil dilatata filiformia, glaberrima; antherce ovatae, biloculares. Ovarium ovatum, biloculare, multiovulatum.. Stylus filiformis, glaberrimus, antherarum altitudine; stigma minutissime capitatum. Capsula ovata, acuminata, calycis tubo aucto dimidio longior, hilocularis, hivalvis, valvaruın apice mox bifidarum margines leviter introflexi, a placenta centrali iisdem parallele compressa soluti. Placenta valvis paullobrevior, scrobiculata. Semina plurima, papaveracea, testa fuscescente, scrobiculata. 
Nr. 9.

\section{NOVARUMITIRPIUI}

\section{DECADES}

\section{Editae a Mluseo Caesareo palatino Vindobonengi.}

Nr. 9. Vindobonae, typis Sollingerianis. 20. Julii 1839.

POLYZONE Endl. Flores supra receptaculum planum, involucratum sessiles, singuli bracteolis geminis, membranaceis, carinatis, distinctis. Calyx tubo obconico, basi cum ovario connato, «onis cartilagineis multiannulato, limbo brevissime quinquefido. Corollae petala 5 , calycis limbo inserta, membranacea, ovata, erecta. Stamina 20 , cum petalis inserta, filamenta brevissima, complanato subulata, alterna ananthera, fertilibus conformia; antherae biloculares, subglobosae. Ovarium inferum, uniloculare. Ovula 2 , basilaria, erecta, extrorsum anatropa. Stylus compressus, apice attenuatus, imberbis; stigma terminale, subcapitalum. Fructus..... - Frutex Novae-Hollandiae austro-occidentalis, ramulis alternis verticillatisque; foliis confertis, acerosotriquetris, capitulis terminalibus, sessilibus, involucri foliolis subcoriaceis, pluriseriatis, coloratis.

Genus Genethyllidi DC. proximum.

81. Polyzone purpurea Endl, in Annal. Wien. Mus, II. 191.

In Novae-Hollandiae austro-occidentalis interioribus, inter Swan-River et King-Georges-Sound (Roë.)

Frutex ut videtur humilis, ramulis oppositis verticillatisque, glabris, inferne foliorum delapsorum cicatriculis torulosis, superne dense foliosis. Folia inbricata, acerosa, subclavato-triquetra, sesquilineam Ionga, dorso acute convexa, facie plana, apice rubentia, obtusa v. mucrone brevissimo calloso terminata, laevia, glaberrima, dorso juxta angulum utrinque punctorum nigricantium seriebus notata. Capitula in apicibus ramulorum solitaria, sessilia, depresse hemisphaerica, diametro semipollicari. Involucri foliola quadri-quinqueseriata, laxiuscule imbricata, extima subherbacea, interiora sensim majora, membranacco-subcoriacea, ovata, obtusiuscula, quatuor lineas longa, duas et dimidiam lineam lata, uninervia, basi decolora, superne pulcherrime purpurea, glaberrima, inte. 
gerrima. Receptaculum subtus convexiusculum, supra planum, minutissime areolatnm, areolis convexis, glaberrimum. Flores in singulis capitulis quindecim ad viginti, sessiles, singuli bracteolis membranaceis geuinis, oppositis, imo calyci insertis, nariculari-carinatis, acutis, uni. nerviis, duas circiter lineas longis, primum marginilus sese amplectentibus inclusi, deinde patentiusculis stipati. Calycis tubus obconicus, basi cum ovario connatus, obsolete pentagonus, unam lineam longus, zonis subcartilagineis, annularibus, senis-octonis, sursum laxe imbricatis, hinc inde interruptis, margine angustissimo, libero, sub lente valde augente incisis vestitus, limbus supra ovarium paullo ampliatus, in tubum dimidia linea paullo longiorem productus, breviter quinquefidus, lobis erectis, obtusis. Petala quinque, submembranacea, laminae calycis tubum intus vestienti, inter ejusdem lobos inserta, ovata, obtusiuscula, basi lata sessilia, uninervia, subcarinata, erecto-conniventia, marginibus sub lente minutissime oerrulata, candida, dimidiam linean longa. Stamina cuin petalis inserta, decem, perfecta cum totidem anantheris, paullo brevioribus alterna; filamenta complanato-subulata, petalis breviora, antherae hiloculares, subglobosae, dorso insertae. Ovarium inferum, vertice complanatum, uniloculare. Ovula 2 , basilaria, erecta, extrorsum anatropa. Stylus subcentralis, ligulato-compressus, basi dilatatus, apice filiformis, sigmoideo-incurvus, breviter exsertus, glaberrimus; stigma terminale, minutissime capitatum, nudum.

THRYPTONENE Endl. Bracteolae 2 distinctae, caducissimae. Calyx tubo cum ovario connato, supra ovarium haud produclo, limbi quinquepartiti laciniis petaloideis, indivisis. Corollae pelalas, calycis limbo inserta, orbiculata. Stamina 10, cum petalis inserta, sterilia nulla; filamenta filiformi-sululala, antherae biloculares, loculis globosis, discretis, e connectivo glandula terminato pendulis. Ovarium inferum, uniloculare. Orula 2 , basilaria, erecta, anatropa. Stylus brevissimus, imberbis; stigma capilatum. Capsula obconica, apice truncata, incomplete bivalvis, abortu monosperma. Semen erectum, exalbuminosum. _ Hruticulus NovaeHollandiae austro-occidentalis ; foliis oppositis, semiteretibus, erectis, mucronatis, pedunculis axillaribus, solitariis, oppositis, unifloris, folio multo brevioribus.

82. 'Thryptomene australis Endl. c. $l$.

In Novae-Hollandiae austro-occidentalis intcrioribus, ad orientem ab urbe New-York legıt cl. Roë.

Rami tetragoni, tenues, glaberrimi, cinerei. Ramuli alterni et oppositi, stricti, semipedales. Folia decussatim "pposita, internodiis trilinearibus sejuncta, subsemiteretia, duas lineas longitudine paullo superantia, erecta, apice patentia, facie plana, dorso convexiuscula, glandulis immersis crebris notata, basi attennata subcompressa, snperne incrassata, apice acuta, in mucronem brevem, rigidum, subcartilagineum, recurvum desinentia. Flores juxta apices ramulorum, in axillis foliorum approximatorum solitarie oppositi, brevissime pedunculati, spicas breves foliosas, v. saepius capitula parva, laxiuscula referentia. Pedunculus brevissinus, vix quintam partem longitudinis folii superans, obsolete angulatus. Bracteolae duae, summo pedunculo insertae, oppositae, lineam 
longae, carinatae, acutae, aequales, fuscescentes, marginibus membranaceis altera alteram amplectens, exterior caducissima, interior plerumque usque ad anthesin, nec tamen diutius persistens. Calycis tubus obconico-teres, lineam longus, glaberrimus, longitudinaliter rugosus, cum ovario connatus, nec ultra illud productus, sllpra ovarium in limbum scarioso-petaloideum, albidum, quinquepartitum expansus, laciniae orbiculatae, lineam longae, paullo latiores, integerrimae, deciduae. Petala 5, laminae carnosae, decoloris, ovarii verticem coronantis margini inserta, calycis laciniis alterna, iisdem aequilonga et conformia, in sicco pallide rosea, patentia, decidua, Stamina 10 , cum petalis inserta, iisdem paullo breviora, omnia fertilia, absque sterilium rudimentis; filamenta filiformi-subulata, antherae biloculares, loculi globosi, e connectivo in glandulam parvain producto penduli, introrsum sulco longitudinali dehiscentes. Ovarium inferum, uniloculare. Ovula gemina, e basi erecta, extrorsum anatropa, horum unum jam in alabastro altero multo minus. Stylus terminalis simplex, glaberrimus, staminibus brevior; stigma capitatum, nudum. Capsula sublignosa, obconica, calycis limbo genitalibusque deciduis vertice plana, incomplete bivalvis. Semen unicum, erectum, testa membranacea, tenui, hinc venosa. Embryo massa homogenea, lactea.

83. Pileanthus peduncularis Endl. l. c. P. pedunculis filiformibus elongatis, filamentis omnibus simplicibus.

\section{In Novae-Hollandiae austro-occidentalis interioribus, inter} Swan-River et King-Georges-Sound legit cl. Roë.

Rami pedales, lignosi, pennae corvinae crassitie, teretiusculi, cortice cinereo, rugoso, glabro. Ramuli alterni et oppositi, juniores brevissimi, foliis dense imbricatis, fasciculum sistentibus velati, adultiores longiusculi, graciles, basi denulati v. foliorum pare uno aliove instructi, apice foliorum confertorum fasciculum brevem gerentia. Folia opposita, in apicibus ramulorum densissime imbricata, clavato suhsemiteretia, obtusa, dorso convexiuscula, facie plana, obsoletissime glanduloso punctata, crassiuscula, glaberrima, vix unam lineam longa. Pedunculi in inferioribus ramulorum terminalium axillis solitarii, uniflori, elongati, racemuın spurie terminalem constitnentes, filiformes, dimidium pollicem $r$. tres quartas pollicis partes longitudine aequantes, supra nonnilil incrassati, glaberrimi, visciduli. Alabastrum pyriforme, tres lineas longum. Bracteolae 2, membranaceae, summo pedicello insertae, marginibus connatae in involucrum undique clausum, superne utrinque mucrone dorsali brevissino instructum, glabrum, viscidulum, pallide citrinum, ad bracteolarum situras utrinque linea rubra notatum, sub anthesi medio circumscissum, calyptra decidua, basi persistente bilineari, campanulata, integerrima, calycis tubum laxiuscule includente. Calycis tubus obconicus, duas lineas longus, fere usque ad mediam longitudinem ovario adnatus, parte supra ovarium producta ampliatus, densissime sericeus, limbus glaberrimus, scarioso-petaloideus, citrinus, decem partitus, lobis obovatis, obtusis, linea paullo longioribus, vix latioribus, margine sub lente obsolete serrulatis, patentissimis v. tandem reflexis. Petala 5, hrud scariosa, ex sicco purpurea, laminae carnosulae ovarii verticem et calycis tubum vestienti inserta, obovato - orbiculata, glaberrima, margine obsolete ciliato - lacera, calycis limbo sextante circiter breviora; vix patentia, caducissima. Stamina 20 , laminae carnosae calycis tubum vestienti continua et concolora, omnia fertilia; filamenta complanato-subulata, petalis breviora, indivisa, decidua, contherae pallide violaceae, ovato-sub 
globosae, dorso supra basim affixae, introrsae, biloculares, loculi appositi, arcte connati, longitudinaliter dehiscentes. Ovarium cum tubi calycini basi connatum, vertice planum, uniloculare. Ovula 3-4, e basi erecta, anatropa Stylus terminalis, filiformis, basi paullo crassior, stamina nonnihil superans, apice subuncinatus, glaberrimus; stigma minute capitatum, nudum.

84. Chamaelaucium virgatuma Endl. $l$. c. Ch. ramis virgatis, foliis oppositis remotis lineari-semiteretibus, pedunculis terminalibus congestis folio brevioribus, bracteolis persistentibus, stigmate barbato.

In Novae-Hollandiae austro-occidentalis interioribus, ad orientem ab urbe New-York legit cl. Rö̈.

Ramulum video bipedalem, virgaeformem, basi penna columbina haud crassiorem, superne sensim attenuatum, apice foliorum fasciculo definitum, inferne ramulos aliquot oppositos, simplicissimos, bi-quadripollicares, strictiusculos, purpurascentes, apice floriferos proferentem, hebetate tetragonum, glaberrimum, epidermide fusca, nitida, lacere secedente vestitum. Folia opposita, paribus internodio octo circiter linearum sejunctis, phyllopodiis prominulis inserta, lineari-semiteretia, glaberrima, internodiis paullo longiora, obtusiuscula, basi nonnihil angustata, dorso convexa, glanduloso-subexaspirata, facie plana, lineae quadrantem lata, strictiuscula. Pedunculi in apice ramulorum inter ultimum par foliorum tres v. quatuor congesti, sesquilineam longi. Bracteolae summo pedunculo insertae, membranaceae, florem primum inclndentes, sub anthesi usque ad tres quartas longitudinis partes patentes, persistentes, ovatae, obtusae, concavae, inaequales, altera interior major, tres lineas longa, quatuor lineas lata, ab altera exteriore paullo minore basi marginibus amplexa, utraque dorso infra apicem in mucronem brevem, rigidiusculum producta, glaberrima, fusca, interioris margines tenerrime membranacei, demum plerumque laceri. Calycis tubus campanulatus, duas et dimidiam lineam Iongus, ima basi cum ovario connatus, Ionge supra illnd productus, glaberrimus, obsolete striatus, herbaceo-viridis; limbi quinquepartiti lobi ovati, obtusi, lineam longi, paullo latiores, integerrimi, glaberrimi, margine nonnihil teneriore rubentes. Petala quinque, summo calycis tubo, ope laminae eundem vestientis inserta, ovata, obtusa, sesquilineam longa, una linea paullo latiora, alba, glaberrima, sub lente minutissime ciliata. Stamina cum petalis inserta, iisdem multo breviora, decem fertilia cum totidem sterilibus ligulaeformibus, integerrimis alternantia; fertilium filamenta lineari-subulata, alterna paullo breviora, antherae biloculares, loculi connectivi glandulaeformis, flavi basi antice adnati. Ovarium cum calycis basi connatum, vix unius lineae longitudine In aequans, vertice planum, uniloculare. Stylus calycis tubo brevior, compressus; stigmate subterminali, barbato. De ovulorum basilarium numero dubius haereo.

85. Kochia salsoloides $F e n z l$. K. fruticosa, diffuse ramosissima, ramis virgatis, cortice cinerascente laevissimo, striato; foliis crasse-filiformibus ( 6 ultimis $2^{\prime \prime \prime}$ longis ac $1 / 2-3 / 4^{\prime \prime}$ latis), obtusis, muticis, patentibus, cum ramulis junioribus incano-pubescentibus, vetulis calvis ; floribus axillaribus, sessilibus, 2 - 7 glo- 
meratis longitudine ramorum remotius confertiusve spicatis, folio bracteali longiore suffultis; perigonii alis transversis obovatosubrotundis, fusco-flabellato-nervosis, tubo puberulo sesquilongioribus.

Patria: Cap. b. Spei. Hab : in Carroa magna ad Jakhalsfontein, alt. $2100^{\prime}$, ec in tervitorio contermino orientali, Koup dicto, prope Beaufort, alt. $2500^{\prime}$ (Drège! collect. p?. cap. n. 8022, $a, c, e, f$.

Fruticis diffusi, procumbentis, ramos video bipedales, crassitie penae corvinae, annotinos cinereos, striatos, juniores dilute testaceos, filiformes, unciales, digitales ac spithameos, conferte foliolatos, tota longitudine florum giomerulis axillaribus onustos, pube canescenti, interdum dilutissime ferruginea, demum fngaci villosulos. Folia teretiuscula, filiformia, mutica, Suaedae frutescentis similia, juniora in apice ramulorwu congesta, pube adpressa incana villosula sive hirsuta, vetula denudata, saepe flaccide reflexa. Perigonium urceolatum, hemisphaericum, extus pubescens $v$. villosulum, quinquefidum, laciniis infra apicem inflexum membranaceum, fimbriarum in alas obovatas, subrotundas, scariosas, transversas, margine undulatas, nervis fuscis copiosissimis flabellatis elegantissime pictas, $1 \frac{1}{2}$ lineam plurimum latas excrescentibus. Stylus bipartitus; stigmatibus setaceis, patentissimis. Seminis horizontalis testa membranacea. Embryo cyclicus, circa albumen parcum curvatus, viridis.

OBSERVATIO. Hochiae prostratae proxima, caudice frutescente, ramis majoribus cinereis, glaberrimis, foliis multo crassioribus, pube omnium partium fugaciore, et perigonii alis majoribus, nervis fuscis flabellato-lineatis diversa.

UNGINADIA Endl. Calyx quinquepartitus. Corollae petala 5, hypogyna, inaequalia, unguibus apice cristatis, erectis, laminis obovatis patentibus. Torus postice laminam erectam, genitalium basim amplectentem exserens. Stamina 9, basi cum tori lamina et ovarii stipite connata; filamenta filiformia, exserta, adscendentia, antherae biloculares. Ovarium stipitatum, triloculare. Ovula in loculis gemina, anguli centralis medio collateraliter inserta, adscendentia. Stylus brevissimus, conicus; stigma simplex. Fructus.... - Arbor boreali-americana; foliis alternis, impari-pinnatis, trijugis, racemis lateralibus corymbosis.

Genus inter Hippocastaneas distinctissimum, anno 1834 dicavi memoriae Davidis ab Ungnad, Rudolphi II. Imperatoris apud Turcas oratoris, qui semina Aesculi Hippocastani Constantinopoli anno 1576 primus Vindobonam ad Corolum Chusium misit. Vid. Clusii Hist. stirp. Pannonic. 3 et 5.

86. Ungnadia speciosa Endlicher Atakta bot, $t .36$.

\section{Habitat in Americae borealis provincia Texas. (Drummond.)}

Arbor magna. Folia alterna!, impari-pinnata, trijuga. Petiolus communis sexpollicaris, pennae corvinae crassitie, facie profunde canaliculatus, dorso convexus, laevis, glaberrimus, inferne crassior, sursum sensim attenuatus, basi obtuse triangulari insertus. Foliola exacte opposita, paribus duorum pollicum intervallo remotis, brevissine petiolulata, ova- 
to-lanceolata, obtuse acuminata, quatuor pollices longa, duos et dimidium pollicem lata, basi rotundata, membranacea, penninervia, obsolete crenulata, supra glaberrima, novella subtus albo-tomentosa, adulta nonnisi ad nerros venasque pilis sericeis subferrugineis parce conspersa, terminale impar petiolo continuum, basi cuneato-angustatum. Ramuli floriferi quos prae oculis habeo, cygneae pennae crassitie, foliorum delapsorum cicatricibns obtuse triangularibus torosi, cortice fusco-rubro, lenticellis discoloribus, raris, ovalibus consperso, gemma multisquamosa obtusa terminati, e gemmarum, immediatim supra foliorum delapsorum cicatribus sitarum, hibernaculis racemos corymbosos plures, pollicares quadripollicaresque, bi-sexfloros, simul com foliis novellis explicantes. Pedicelli fere filiformes, pilis sericeis testaceis hirti, infra medium articulati, ibique densius pilosi, laxe patentes. Calycis usque ad basim, toro plano antrorsum declivi adnatam, quinquepartiti laciniae lanceolatae, acuminatae, herbaceae, venosae, extus imprimis basi pilosae, tres circiter lineas longae, lineam latae, planae, postica nonnihil profundius distincta, erecta, laterales cum anticis vix quidquam majoribus paullo altius cohaerentes, ob basim declivem antrorsum patentiusculae. Corollae petala 5 , toro plano inter calycis lacinias inserta, duo postica erectiuscula, lateralia cum antico paullo minora, omnia unguiculata, ungues carnosuli subcuncati, dense albo-tomentosi, sesquilineam longi, apice crista e filis in omnem sensum patentibus, teretiusculis, carnosis, flexuosis, apice saepissime glandula globosa terminatis ornata, lamina rubra, obovata, obtusa, subtilissime crenata, tenera, venosa, dorse glandulis resinosis conspersa. Lamina carnosa e medio toro exserta, calycis laciniae posticae opposita, erecta, obtusa v. emarginata, marginibus stamina et ovarii stipitem amplectens, partimque eorundem basibus adnata. Stamina 9 , inaequilonga, hornm duo postica, juxta laminae apicem exserta, petalis duolus posticis respondentia, reliquis longiora, duo utrinque calycis laciniis lateralibus petalisque lateralibus opposita ab eadem lamina amplexa, duobus ante calycis lacinias anticas sitis, laminae extremis marginibus contiguis subaequilonga, nonum petalo antico oppositum, a lamina liberum, ima basi staminibus contiguis et gynophoro cohaerens, omnia longe exserta, antrorsum declinata et adscendentia; filamentis filiformibus, sursum attenuatis, glaberrimis, ex sicco violaceis, antheris bilocularibus, ovatis, obtusis, pilosiusculis, longitudinaliter dehiscentibus. In nonnullis floribus ovario brevius stipitato donatis, antherae fortassis efoetae, polline certe in speciminibus examinatis destitutae. Ovarium centrale stipite plns minns longo, nunc laminae posticae longitudinem vix aequante, nunc superante, inferne cum staminibus et eadem connato sustentum, ovato-subglobosum, dense piloso-setosum, triloculare. Ovula in loculis gemina, anguli centralis medio collateraliter inserta, adscendentia. Stylus brevissimus, subconicus, obtusus, obsolete trisulcus. Fructus haud suppetit.

8\%. Jacksonia foribunda Endl. in Annal. Wien. Mus, II. 197. J. ramulis phyllodineis oblongis basi cuneatis sinuato-dentatis glaberrimis utrinque venosis, floribus racemosis, calycibus sericeis corolla longioribus, leguminibus stipitatis.

In Novae-Hollandiae austro-occidentalis interioribus, inter King-Georges Sound et Swan-River. (Roë.)

Species distinctissima, inter congeneres facile pulcherrima, $J$. dilatatae Benth. proxime affinis. Frutex, si e speciminibus recte judico, caespitosus, ramis sesquipedalibus bipedalibusque, e basi procumbente adscendentibus, pennae anserinae crassitie, sulcato-striatis, basi subglabris, 
inferne teretibus, superne densius pubescentibus, sensim compressiusculis, apice breviter bi-trifidis, foliace-compressis. Ramuli alterni, pollicem v. sesquipollicem dissiti, phyllodinei, crassiuscule coriacei, horizontales, duos v. quatuor pollices longi, dimidium pollicem lati, basi cuneati, in petioli speciem angustati, apice acuti v. subtruncati, ambitu remote sinuato-dentati, dentibus patentibus v. subrecurvatis, prima juventute folio squamaeformi terminatis, mox callosis, acutis, rarius in ramulum secnndarium, aeque sed directione contraria dilatatum excrescentibus, $v$. in rami parte superiore in ramulum floriferum productis $v$. simpliciter unifloris, ramulo secundario sive florifero, sive sterili semper folio squamaeformi diutius persistente, v. saltim ejus cicatrice basi stipato. Ramulorum phyllodineorum pagina utraque prima juventute pilis sericeis, adpressis canescens, mox glabrata, pallide virens, nervus primarius validior, subtus subconvexus, superne obsoletus, secundarii utrinque aeque manifesti, alterni, arcuati, nonnulli paullo validiores, dentes superne marginantes, omnes in rete areolatum coeuntes, areolarum disco immerso, sub lente minutissime puberulo. Folia vera ad basim ramulorum et juxta eorundem dentes squamaeformia, coriacea, ovata, acuta, subcarinata, duas lineas longa, sesquilineàm lata, fusco-nigra, pubescentia, decidua, ad basim ramulorum cicatricem semicircularen relinquentia. Flores in inferiore caulis parte ramulorum dentes hine inde solitarie terminantes, in apice caulis racemos breves, compositos formantes. Pedunculi basi teretes v. compressi, alternatim ramosi, ramulis compressis v. uno alterove foliaceo dilatato, sericeo-canescentibus. Pedicelli uniflori, tetragoni, fulvo-sericei, basi folio coriaceo squamaeformi, apice bracteolis geminis subcoriaceis, sericeis, ovato acuminatis, bilinearibus muniti. Alabastra semipollicaria, ovato-subconica, calycis laciniis valvatim applicitis pentagona, acuminata, fulvo-pubescentia. Calycis quinquepartiti tubus brevissimus, vix dimidia linea longior, laciniae coriaceae, lanceolatae, acuminatae, septem lineas longae, sesquilinea paullo latiores, aequales, uninerves, extus pube sericea, sordide flava $v$. subfulvescente villosae, apice acuminato intensius coloratae, subsphacelatae, intus glabrae, aestivatione marginibus incrassatis valvatim cohaerentes, sub anthesi laxiuscule campanulatae. Corolla papilionacea, calyce paullo brevior. Vexillum late obovatum, basi subcuneata brevissine unguiculatum, apice fere ad tertian longitudinis suae partem emarginato-bilobum, ex sicco aurantiaceum, inferne sordide violaceum, flabellato venosum, sex lineas longwm, tres et dimidiam lineam latum, si ex sicco recte judico sub anthesi patentiusculum. Alae oblongae, vexillum aequantes, unam lineam latae, superne aurantiaceae, inferne pallidiores, apice obtusae, latere antico subtruncatae, basi postice unguiculatae, antice in auriculam minimam, acutiusculam sursum productae. Carina alis aequilonga, genitalia includens, petalis medio cohaerentibus, basi bipes, apice incurva, obtusa. Stamina 10, laminae carnosae, calycis tubum brevissimum intus vestienti et annulo brevissimo marginanti continua; filamenta filiformi-subulata, libera, glaberrima, corolla paullo breviora, antica reliquis nonnihil longiora, persistentia, antherae ovato-oblongae, dorso supra basim affixae, biloculares. Ovarium pedicello calycis tubum paullo superanti impositum, sericeovillosissinum, biovulatum. Stylus filiformis, staminum longitudine, apice incurvus, glaberrimus; stigma terninale, minutissime capitatum. Legumen (quale petalis styloque jau delapsis nondum penitus maturum examinavimus) stipitulo unam lineam longo impositum, ovato-orbiculatum, turgidulum, compressum, tres lineas longm et latum, extus densissine sericen-villosum, valvulae coriaceas, intus adpresse puberulae. Ovula 2 , contigue superposita, in funiculis latiusculis in mediam cavitatem prominentibus excentrice amphitropa, subreniformia, estrophiolata, inferius plerumyue multo minus evolutum, unde legumen abortu monospermuas conjecturari potest. 
GIISCHROCARYON Endlicher Gen. plant. Suppl. $\frac{703 x}{1}$. Flores dioici. Masc. ..... Fem. Perigonium tubo clavato-tetraptero, cum ovario connalo, alis in pedicellum subdecurrentibus, cum limbi superi, quadripartiti, persistentis lobis brevibus, obtusis alternantibus. Discus epigynus nullus. Stamina sterilia 12, perigonii lobis basi ternatim inserta, filiformi-subclavata. Ovarium inferum, uniloculare. Ovula 4 , ex apice placentae filiformis, liberae pendula. Stylus brevissimus; stigmata 4 , exserta, oblongoclavata, erectiuscula. Drupa baccata, clavato-tetragona, membranaceo quadrialata, sarcocarpio fibroso spongioso, endocarpio cartilagineo, monospermo. Semen inversum. Embryo in apice albuminis carnosi brevis, rectus, radicula supera. - Suffrutex Novae-Hollandiae austro-occidentalis ; foliis lineari-lanceolatis, integerrimis, racemis terminalibus corymbosis, ebracteatis.

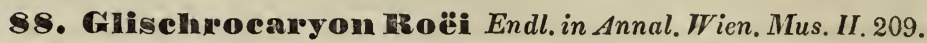

\section{Crescic in Novae-Hollandiae austro-occidentalis interiori- bus (Roë).}

Ramorum summitates video pedales, teretes, pennae corvinae crassitic, glaberrimas, striatas, laeves, sordide violaceo-virescentes. Folia in summitatilus mihi obviis pauca, alterna, duos pollices dissita, lanceolata, utrinque angustata, acuta, sex v. octo lineas longa, lineam lata, integerrima, glaberrima, supra pallide viridia, subtus glauca. Racemi terminales alterni, pedunculi teretes, stricti, subcorymbosi, pollicares, pedicellis quinis v. denis filiformibus, duas ad quatuor lineas longis, in apice pedunculorum corymbose confertis. Perigonium sesquilineare, glaucum, tubo clavato - tetraptern, cum ovario connato, alis suturalibus angustis, membranaceis, cum limbi quinquepartiti laciniie brevissimis, obtusis, erectis alternantibus. Stamina sterilia 12 , perigonii laciniis basi ternatim inserta, iistem paullo longiora, filiformi-subclavata. Ovarium inferum, uniloculare. Ovula 4, ex apice columnulae subtilissime filiformis, in stylum continuae pendula. Stylus brevissimus; stigmata 4, oblongo-clavata, erecta, approximata, perigonio duplo longiora. Drupa laceata, viridiglauca, Pisi mole, clavato-tetragona, alis quatuor membranaceis, dimidiam circiter lineam latis, usque in medium pedicellum decurrentibus, sensim angustatis, limbi lobis conniventibus, stylisque persistentibus co-: ronata. Sarcocarpium valide fibrosum, fibris ab endocarpii pariete externa versus peripheriam radiantibus, reticulo spongioso tenaci combinatis. Endocarpium ovatum, lineam longum, intus nitidum. Semen ex apice fili tenuissimi, rigidi, parieti hinc appliciti, sed ab eadem liberi, basi et apice inserti pendulum, cavitatem implens, apice carpico attenuatum, testa membranacea, fusca. Embryo in apice carpico albuninis inclusus, vix tertiam ejusdem longitudinis partem aequans, rectus, cotyledonibus brevibus, obtusis, radicula supera.

CYCNOGETON Enalicher Gen. plant. Suppl. n. $\frac{1664}{1}$. Flores hermaphroditi, spicati. Perigonium calycinum, hexaphyllum, foliolis sessilitus, aestivatione valvatis, sub anthesi patentibus, deciduis. Stamina 6 , perigonii foliolis adnata, antherae biloculares, loculis oppositis, discretis, longitudinatiter dehiscentibus. Ovaria 6, libera, sessilia, dorso convexa, a latere compressa, 
faciebus contigua, unilocularia. Ovula 2, suturae ventrali prope basim contigue superposita, anatropa, superius minus. Stylus terminalis, brevis, intus stigmatosus. Nuculae 6, compressae, falcalae, putamine coriaceo, monospermae. Semen falcatum, testa membranacea. Embryo exalbuminosus, macropodus, homotropus, extremitate radiculari infera. _ Herba in fluvio Cygnorum Novae-Hollandiae austro-occidentalis vegetans, tripedalis; foliis gramineis basi vaginante scapum simplicissimum amplectentibus, spica terminali stricta, laxiflora.

Genus inter Potamogetonem et Ouvirandram (coll. Decaisne in Delessert Ic. select. III. p. 62. t. 99. 100. Endlicher Gen. plant. Suppl. $\frac{1664}{2}$ ) medium.

\section{Cycnogeton IItigelii Endl. Iconogr. $t$. 73.} Hïgel.

In fluvio Cygnorum (Upper-Swan-River) legit Carolus L. B.

Folia graminea, acuta, strictiuscula, tripedalia, dimidium pollicem lata, longitudinaliter striata, acuta, glaberrima, laevia, basibus dilatatis scapi dimidiam partem inferiorem vaginatim amplectentia. Scapus simplicissimus, strictus, compressinsculus, striatus, foliis paullo longior, apice in spicam circiter pedalem, interrupte multifloram desinens. Pedicelli geminatim ternatimve approximati, filiformes, lineam longi, floriferi patentes, fructiferi strictiusculi, ebracteati. Flores ut e sicco videtur fuscescentes. Perigonii foliola sex, sessilia, oblonga, obtusa, vix dimidiam lineam longa. Filamenta brevissima, basi laciniarum perigonii adnata, antherae terminales, perigonii foliolis paullo latiores, loculis oblongis, suboppositis, connectivo interposito subdiscretis, longitudinaliter dehiscentibus. Ovaria sex, sessilia, libera, compressa, faciebus subconniventia, in stylum brevem, intus albido-glandulosum continua, unilocularia. Ovula 2 , suturae ventrali paullo supra basim inserta, contigue superposita, anatropa, funiculis brevibus subtensa, alterum superius minimum, alterum inferius maximum, fere cavitatis longitudine. Nuculae sex v. abortu interdum pauciores, coriaceae, falcatae, turgidulae, semicirculum radii trilinearis fornantes, medio unam lineam latae, utrinque angustatae, apice intus unius lineae longitudine stigmatosae, dorso acute convexae, lateribus utrinque uninerves, sutura ventrali subincrassata. Semen ovuli superioris abortu constanti unicum, homotrope arcuatum, suturae rentrali paullo supra basim affixum, teres, testa membranacea, umbilico prope basim introrsum laterali, rhaphe filiformi, juxta interius seminis latus procurrente, in chalazam introrsum terminalem, obscurius coloratam desinente. Plumula brevissima, cotyledonis basi adpressa, extremilatis radicularis infera.

D0. Souriangia oppositifolia Reissek. S. foliis decussatim oppositis ovali-subrotundis, supra glabris nitidis, subtus albo tomentosis, stipulis villoso-cristatis, cymis axillapibus paucifloris, pedicellis oppositis.

Plyylica cotinifolia Portenschlag msc.

Habilat in insula St. Helenae. (Wolley in Herb. Horlen. schlag.) 
Species habitu insignis. Fruticis (?) ramuli inclinati, subangulatostriatuli, verruculosi, fusci, adultiores glabri, juniores pennae corvinae crassitie, sursum villo sordide albido, denso, patente, serius cinereo, evanescente tecti. Petioli quinque v. sex linearum intervallis distantes, decussati, exacte oppositi, duas v. tres lineas longi, patentes, hirsutovillosi, villo ut in ramulis deciduo, crassi, cicatrices conspicuas relinquertes. Folia sesquipollicen longa, ultra pollicem lata, ovalia v. subrotundo-ovalia, plerumque utrinque rotundata, nonnulla basi paululum in petiolum producta, integerrima, subundulata, coriacea, adultiora plana, supra glaberrima, nitida, dense impresso-reticulata, subtus costata, costa primaria valida, hirsuto-tomentosa, pilis simplicibus flexuosis laxe convolutis, inter costas obsolete reticulata, juniora marginibus revoluta, supra basi et in sulcis costarum albido-tomentosa, novella usque ad costam revoluta. Stipulae duas lineas longae, ovato-lanceolatae, earinatocompressae, subundulatae, subcrenatae, acutae, pallide bruneae, dorso dense villoso-cristatae, mox deciduae. Pedicelli in pedunculis communibus axillaribus, pedicello fere duplo longioribus pauci, breves, oppositi, conferti, cymosi. Calyx lineam fere longus, abconicus, sursum ampliatus, in lacinias patentes, acutas divisus, extus pilis simplicibus flexuosis, hygrometricis hirsutus. Petala parva, integra, cucullata, brevissime unguiculata, brunea, margine albescente. Stamina petalis inclusa ; filamentis brevibus, basi dilatatis, vix incurvis. Antherae reniformes, nniloculares, bivalves. In floribus abortivis staminum loco fasciculus pilorum brevium, albidorum, simplicium. Discus crassus, carnosus, subconvexus, ovarii verticem tegers. Ovarium fundo tuboque calycis adnatum, triloculare, ovulis in loculis solitariis, erectis, minimis. Styli tres, breves, crassiusculi, sursum attenuati, subdivergentes; stigmata simplicia. Fructus duas lineas longitudine superans, ovatus, semiinferus, medio limbi calycini reliquiis cinctus, trilocularis $\mathbf{v}$. abortu bilocularis, tri-dicoccus, coccis indehiscentibus, coriaceo-lignosis. 
Nr. 10.

\section{NOVARUII STIRPIUII}

\section{DECADES}

\section{Wuitae a Iriseo Caesareo palatino Vimetobonersi.}

Nr. 10. Vindobonae, typis Sollingerianis. 5. Augusti 1839.

SCHWABEA Endlicher Gen. plant. Supplem. n. $\frac{4072}{2}$. Calyx ad basim quinquepartitus, lacinia antica paullo latiore. Corolla hypogyna ringens, fauce antice ventricosa, postice intus biappendiculata, labio superiore erecto subfornicato, emarginato, inferioris longioris patentis trifidi lobis obtusis. Stamina 4, corollae fauci insertu, sub labio superiore adscendentia, didynama; antherae loculis inaequalibus, confluentibus, basi barbulatis subuniloculares. Ovarium biloculare, loculis uniovulatis. Stylus simplex, stigma bilobum. Capsula chartacea, bilocularis, loculicide bipartibilis. Semina compressiuscula, retinaculis late cymbiformibus subtensa, testa humectata in vasa spiralia soluta. - Herba Africae tropicae. orientalis annua, simplex; nodis turgidis, foliis oppositis, ellipticis, integerrimis, floribus axillaribus solitariis, sessilibus.

Genus Acanthaceum, soli Russeggerae, habitu diversissimae affine, et novae tribus pigruus, dico honoribus viri clarissimi et perillustris Domini Henrici Schwabe, Dessaviensis, acutissimi plantarum, imprimis Algarum, indagatoris.

\section{Schwabea modesta Endl.}

Habitat in Africa tropica orientali. (Kotschy.) Colitur in horto Hügeliano.

Herba annua, caule in speciminibus cultis pedali et sesquipedali, tenero, debili, erecto, simplicissimo, tetragono, ad nodos tumidos et juxta angulos pilis brevibus, rigidulis consperso. Folia opposita, paribus in inferiore caulis parte internodiis bipollicaribus sejunctis, superne brevioribus, brevissime petiolata, elliptica, utrinque angustata, acutiuscula, inferiora pollicem v. sesquipollicem longa, superiora bipollicaria, vix dimidium pollicem latitudine aequantia, penninervia, nervis incurvis, intra marginem combinatis, integerrina, utrinque pallide viridia, subtus fere glaucescentia, ad nervos in utraque pagina, imprimis tamen in inferiore pilis hyalinis conspersa, margine prope basim ciliolata. Flores in 
superiormm foliorum axillis solitarii, sessiles, oppositi. Bracteolae 2 , herbaceae, anticae, lineari lanceolatae, tres et dimidian linean longae, patentes, margine pilis longis, paveis, complanatis hyalinis hispidulae. $\mathrm{Cu}$ lycis usque ab basim quinquepartiti laciniae lanceolatae, longe cuspidatae, herbaceae, tres lineas circiter longae, margine ciliatae, cuspidibus fere aequilongis, basi pilis longis patentiusculis rigidulis, raris barbato-hispidulis, una antica paullo latior, vix longior, cum duabus lateralibus duas posticas erectas longitudine et latitudine aequantibus, apice patens, Corolla calyce paullo longior, tres lineas longitudine parum superans, v. tres lineas et dimidiam aequans, bilabiatim ringens, tubo albo, sursum ampliato, antice ad faucem inflato gibboso, postice ad labii superioris exortum infra inferioris commissuram utrinque impresso, intus in lobulos triangulares deflexos, liberos, fancem claudentes, barbato-ciliatos producto. Labium superius tubi longitudine, late ovatum, subfornicatum, erectum, apice brevissime emarginatum, dilute stramineum, lineo. lis sex longitudinalibus, inaequalibus, interruptis, inferne saturatius, superne dilutissime et sordide purpureis pictum, basi plicis transversis in lobulos supra descriptos productis constrictum. Labium inferius superiore quadrante circiter longius, patentissimum, obovatum, breviter trifidum, lobis obtusissimis, intermedio vix quidquam longiore, onnibus integerrimis, pallidissime flavis, palato biplicatum, plicis elevatis laete purpureis, rictu fasciculis pilorum albicantium clauso. Stamina 4, summae corollae fauci inserta, intra lahium superius recondita, duo postica inter corollae labium superius et inferioris lobos laterales inserta, eidem aequilonga, erectiuscula, duo ad basim labii inferioris suturae qua lobus intermedius cum lateralibus cohaeret, utrinque respondentia, paullo breviora, conniventia. Filamenta lineari filiformia. Antherae loculis valde inaequalibus, altero plus dimidio breviore, apice confluentibus subuniloculares, bicrures, cruribus e connectivo dependentibus, rima confluente apertis, basi barbulatis. Ovarium ovatum, sessile, disci lamina biloba rotundata, anticum et posticum ab axi latus occupante, adpressa stipatum, biloculare. Ovula in loculis solitaria, utrinque retinaculo late cymbiformi, apice libero barbato, paullo supra loculi basim inserta. Stylus filiformis, apice incurvus, brevissine bilobus, lobis obtusissimis, planiusculis, hiantibus, inferiore paullo longiore. Capsula, cujus nonnisi fragmenta suppetunt, ut videtur brevissime unguiculata, chartacea, bilocularis, Acantbacearum more loculicide bipartibilis. Semina in loculis solitaria, compressiuscula, testa humectata in vasa spiralia innumera cum impetu fatiscente.

PETALOPOGON Reisseck. Calyx tubo subcampanulato, basi cum olario connato, superne libero, limbi quinquepartiti laciniis basi inflexis, gibbosis, conniventibus, gibbis barbatis, faucem claudentibus. Potala 5, medio calycis lubo inserta, conchalo-cucullata, ciliato-barbata. Stamina 5 , cum petalis inserta, iisdem opposita, filamenta brevissima, incurva, antherae reniformes uniloculares, rima arcuaca bivalves. Discus tenuis, calycis tubumet lacinias tegens. Ovarium inferum, triloculare, loculis uniovulatis. Stylus simplex, brevis; stigma capitatum. Fructus inferus, ovatus, areola parva notalus, stylo persistente superatus, tricoccus, coccis crustaceis. Semina podospermio cupulato, lobulato sulfulta. - Fruticulus capensis, Phylicae facie; ramis fastigiatis, foliis sparsis, cordato-cuspidatis, margine revolutis, subtus lanatis, exstipulatis, floribus capitato-spicalis, villosis, bracteis foliaceis, florem subaequantibus. 
Genus calyce clauso, et petalis staminibusque medio tubo insertis, egregie inter Rhamneas distinctum.

\section{Hetalopogon cuspidatus Reisseck.}

Phylica cuspidata Ecklon et Zeyher Enumeratio plant. Afric. austr. I. 134.

Habitat in Capite bonae Spei. (Drège no. 6754.) - In sabulosis altitudinis primae et secundae districtus Worcester, prope Bergualley. (Ecklon no. 1031.)

Frutex humilis, ramis teretibus, dimidiam linean diametro aequantibus. Ramuli subumbellato-fastigiati, e basi capitulorum annotinorum deliquescentium provenientes, subpatentes, in sicco striatuli, epidermide brunescente, villis simplicibus, patentibus, superne pilis nigrescentibus mixtis, in junioribus densioribus, serius sensim evanescentibus cani. Folia sesquilineam v. duas lineas longa, persistentia, sparsa, conferta, erectopatentia, in ramulorum apicibus laxe subimbricata, breviter petiolata, e basi latissima sesquilineam aequante fere sagittato cordata, lobis revoluto-complicatis, obtusis, subtus concavis, in cuspidem recurviusculum angustata, marginibus integerrimis revoluta, inferiora superne glabra, nitida, minute flavescenti verruculosa, scabriuscula, superiora veriucis piliferis canescentia, omnia subtus costa conspicua percursa, et cum petiolis pilis tenuissimis, flexuosis tomentoso-lanata. Stipularum vestigium nullum. Capitula florum spicaeformia, tres v. quatuor lineas longa, subrotundo-ovata, densa, octo-ad sedecimflora. Bracteae sub floribus singulis singulae, ejusdem longitudine, ovato-lanceolatae, hirsutae, raeterum foliis conformes, ita ut flores singuli quasi axillares. Pedunculi brevissimi, teretes, hirsuti, plerumque bracteola unica, setacea, tenuissima, villosa, calycis dimidio breviore muniti. Calyx lineam longus v, paullo longior, tubo subámpanulato, basi angustata cum ovario penitus con. nato, supra medium in lacinias quinque diviso, extus praesertim basi villis densis patentiusculis albis hirsutus, intus glaberrimus, brunescens. Laciniarum bases triangulares, repente constrictae, conniventes, gibbosae, gibbis fasciculis pilorum flavicantium, brevium barbatis, tubum arcte claudentibus, superne in cuspides erecto-patentes triquetras, apice subincurvas, intus glabras attenuatae. Corollae petala 5, exacte medio calycis tubo inserta, octavam lineae unius partem longitudine metientia, erecta, breviter unguiculata, in laminam conchatocucullatam, glaberrimam, margine antico pilis longis, rigidulis, erectis, flavescentibus dense ciliato-barbatam, bruneam expansa. Stamina 5, petalis opposita. Filamenta basi crassiore dilatata, unguibus concreta, brevia, teretia, supra incurva. Antherae $1 / 10$ lin superantes, cordato - reniformes, laeves, glabrae, loculis duobus parallelis confluentibus uniloculares, rima arcucata antice bivalves, valvis polline emisso inflexis, postice pro filamenti insertione foveolatis. Discus nec distinctus, nec limitatus, calycis tubum et lacinias strato tenui, carnoso tegens. Ovarium inferum, calycis fundo immersum et adnatum, obovatum, supra planum, triloculare, loculis parvis, uniovulatis. In floribus abortivis ovarii loco pilorum fasciculus. Ovula ex angulo interiore adscendenti erecta, ovata, obtusa, laevia, anatropa, basi intus ad dissepimentum micropyle aperta. Stylus longitudine ovarii, simplex, crassus, teres, stigmate capitato, subtrilobo, papilloso terminatus. Fructus fere duas lineas Iongus, inferus, ovatus, obtuse trigonus, supra areola parva, rotunda notatus, stylo brevi, persistente superatus, parce tomentoso lanatus, siccus, epicarpio tenuissimo, membranaceo, subfnsco, reticulato, trilocularis, tricoccus, coccis primu in arcte cohaerentibus, dein rima interna longitudinali dehiscentibus, crustaceis, flavescentibus, monospermis. Semina erecta, obovata, trigona, dorso subconvexa, obtusa, Jaevia, po- 
dospermio parro, ad basim usque in lobulos radiatos, corneos, obtusos diviso suffulta. Albumen ....Embryo rectus, magnus, cotyledonibus planis, sibi invicem applicitis, radicula brevi, obtusa, infera.

MONOCOSMIA Fenzl. Calyx disepalus, persistens, sepalis ovali-subrotundis, obtusissimis v.retusis, dorso in alam sacciformem certicaliter compressam protrusis, herbaceis. Petala 3, rarissime 4, aequalia, hypogyne inserta, libera, oblonga, integerrima, post anthesim yelatinose confluentia, apice contorta, ovarium diutius coronantia. Stamen unicurn, petalo oppositum. Ovarium uniloculare, ovulis 2 v.4, duobus tunc primitus hebetioribus, omnibus basifixis. Stylus brevissimus, bifidus, stigmatibus abbreviatis, patentibus. Capsula membranacea, bivalvis, mono-disperma. Semina lenticulari compressa, obtuse granulata, lucida, estrophiolata. Herba chilensis, annua (?), multicaulis, succulenta, glabra, folia radicalia rosulata, lamina deltoideo-rhombea, obtusa, in petiolum late linearem longissimum attenuata, caulina alterna, exstipulata. Flores minulissimi, brevissime pedicellati, per racemos axillares et terminales, inferius foliatos, superne aphyllos dispositi, subsecundi, conferti.

OBSERVATIO. Genus inter Calandriniam, Claytoniam et Calyptridium ambigens, cum priore Calandriniae aptius fortassis consociandum, a Calyptridio corolla polypetala et ovulorum numero definito, a Claytonia flore monandro, tripetalo ae stylo bifido, a Calandrinia iisdem characteribus et ovulorum numero stigmatibus aequali v. solum duplo, recedens.

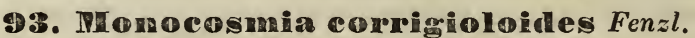

Talinum monandrum Ruiz et Pavon Prodr. 65. Syst. 116. - Calandrinia monandra DC. Prodr. III. 359. Corrigiola deltoidea Pöppig Plant. chil. n. 91. Diar. n. 650.

Patria: Chile. Habitat: in collibus aridis Conceptionis prope fluvium Andalien (Ruiz et Pavon.) - In arenosis montosis ad Tumbes Talcahuanensium (Pöppig!)

Herba annua (?), multicaulis, glaberrima, succulenta, laete virens. Caules in orbem diffusi, simplices $\mathbf{v}$. inferius abbreviato-ramosi, digitales ac palmares, crassitie fili emporetici mediocris v. tenuioris, angulati, foliosi, tota longitudine florum racemulis alternis, plus minusve remotis obsessi, apice longioribus tribus ad quinque subcymose confertis terminati. Folia radicalia confertissime rosulata, 1_3-uncialia, carnosa, uninervia, lamina deltoidea, basi rhombea, $3-8^{\prime \prime \prime}$ solum longa et $2 \ldots 5^{\prime \prime \prime}$ lata, angulis apiceque obtusissimis, in petiolum linearem, $1 / 2$ "' latum attenuata; caulina radicalibus similia, sensim minora, demumque lineari oblonga v. lanceolata, alterna, exstipulata, carina marginibusque in angulos decurrentia. Racemi $1 / 2-1 \frac{1}{2}$ unciales, inferius foliolosi, superius aphylli, virginantes subscorpioidei. Flores minutissimi, vix magnitudine Corrigiolae vulgaris, in racemis subsecundi, primum confertissimi, demum remotiusculi, nutantes, pedicellis $1 / 2-2^{\prime \prime \prime}$ longis suffulti. Calyx disepalus, persistens, herbaceus, $1 / 2$ _ $1^{\prime \prime} \lg$., sepalis ovali-subrotundis, concavis, apice plurimum retusis, medio dorso $v$. infra apicem in alam v. gibbum sacciformem, verticaliter compressum protrusis. Petala 3 , rarissime 4 , calyce subbreviora, late linearia v. oblonga, obtusa, basi libera, tenerrima, 
alba. Stamen solitarium, petalorum longitudine, illorum uno basi hypogyne insertum. Anthera ovalis, bilocularis, versatilis. Stylus minutissimus, bifidus, ramis stigmatosis brevissimis, patentibus. Ovarium plurimum 2-, rarius 4-ovulatum, ovuloram tunc semper duo imperfectiora, omnia basifixa. Capsula ovoidea, calycem parum superans, tenue membranacea, bivalvis, 1_2sperma. Semina lenticularia, atra, lucida, sub lente granulis complanatis dense consita, diametro $1 / 2$ '" lata.

ANCISTROSTIGMA Fen:l. Calyx campanulatus, ultra medium 5-fidus, laciniis obtusis, duobus interioribus latioribus semiscariosis, concaviusculis. Corolla nulla. Stamina plurimum $7-8$, rarissime 9 , seriei primae quinque sinubus, secundae duo v. tria calycis laciniis internis et tertiae semiexternae e diametro v. lateraliter opposita, subaequilonga ac calyce parum breviora. Antherarum loculi lineari-oblongi, infra apicem solum coaliti, caeterum liberi. Ovarium calycis tubo immersum, liberum, 1-loculare, pluriovulatum, columella centrali libera, ovulis funiculis umbilicalibus distinctis adscendentibus affixis. Stylus unicus, centralis, sigmoideus, apice uncinato recurvus, facie exteriore curvata stigmatosus. Capsula subglobosa, membranacea, medio circumscisse dehiscens, 5-8-sperma. Semina reniformia, lucida, atra. - Herba Novae-Hollandiae extratropicae orientalis, glabra, caudiculis perennantibus humifusis, varie ramosis, habitu Cypseleae v. Herniariae. Folia parva, ovalia, in ramulis conferta, alterna et opposita, petiolis stipulaceo-vaginantibus. Flores axillares solitarii, breve pedicellati, minimi.

Obs. Genus Cypseleae proximum; staminum numero, ac stylo elon. gato, simplicissimo, indiviso, sigmoideo - uncinato diversum; Trianthemati, calyce 5-partito, stylo simplici, habitu et stipulis indivisis petiolaribus affine, ovario libero uniloculari, intus dissepimento transverso spurio non intercepto, aliisque notis abunde recedens.

\section{Ancistrostigma eypseleoides Fenzl.}

Patria: Nova Hollandia orientalis extratropica. Habital: cul Hawkesbury-River, $30^{\circ} 15^{\prime}$ L. A. (Ferd. Bauer!)

Herba perennis, habitu Herniariae magis quam Centunculi, Cypseleue humifusae caeterum valde accedens, glaberrima, ramosissima, humo adpressa. Caudiculi filiformes, crassiusculi, digitales, lignescentes ac partibus vetustioribus demum radicantes, ramulis florigeris uncialibus, semiuncialibus ac minoribus, dense foliatis, in caespitem planum confertis onusti. Folia distincte petiolata, opposita et alterna, in axillis juniora 2 ad 5 cum fosculo simul foventia, lamina ovali-subrotunda $v$ ovata, plana, carnosa, uninervia, obtusissima, integerrima, $1-2^{\prime \prime \prime}$ longa et $1 / 2-1^{\prime \prime \prime}$ lata, in petiolum tenue filiformem, dimidio breviorem, basi membrana stipulari, scariosa, in denticulum utrinque producta auctam, semiamplexicaulem haud attenuata. Flores minuti, foliis breviores, axillares, solitarii, pedicellis demum calycem aequantibus, frequentes. Calycis $3 / 4-11 / 3^{\prime \prime \prime}$ longi, glaberrimi, subcampanulati, ultra medium quinquefidi, laciniis in aestivatione exterioribus ovatis, interioribus ovalibus obtusissimis, nedio herbaceis, reticulato venulosis, margine scariosis, concaviusculis, viri- 
dibus, tubo infundibuliformi teretiusculo. Stamina plurimum 8 , raro 7 v. 9 , summo calycis tubo inserta, 2 v. 3 laciniis interioribus $\mathbf{v}$. semiexternae, florum ennandrinm unicum externarum unae e dismetro v. (quod frequentissime) lateraliter oppositum, calyce subbreviora, subaequilonga. Antherarum loculi lineari-oblongi, didyni. Ovarium liberum, globosum, 1loculare, 6_120rulatun, orulis columellae centrali liberae funicnlis distinctis affixis, amphitropis, adscendentibus. Stylus centralis, signoideus, apice uncinatus, filiformis, facie exteriore stigmatosus. Capsula ellipsoidea, membranacea, medio circumscisse dehiscens, columella centrali persistente. Semina 4_8, reniformia, laevissima, extremitate radiculari solam subquinquestriata, lucida, atra, $1 / 3^{\prime \prime \prime}$ lata.

95. Cisecinia IIItus Fenzl. G. annua, glabra; multicaulis, dichotome ramosa ; foliis lineari-oblongis, oblongis v. subspatluulatis, obtusissimis, petiolatis v. subsessilibus, oppositis, alternis v. pseudoverticillatis, subtus deusissime granulatis; cymulis umbelliformibus axillaribus, sessilibus v. pedunculatis; calycis laciniis late ovalibus, obtusissimis; staminibus 10_15, per phalanges 2_3stemones inter lacinias calycis insertis; carpidiis nuricatis, ambitu cristatis, purpureis.

Miltus africana Loureiro cochin. 1. 369. DC. Prod. 3. 45 t. Glinus mozambicensis Spr. Syst. 2. 467.

Patria. Africa australis. Hab. in locis aridis insulae Mo:ambique (Loureiro.); ad ripas amnis Garip, lateris occidentalis, infra 300' (Drège! collect. pl. cap. n. 3019). Fl. Sept.

Herba diffusa, glabra, habitu subinde suffrutescente, annua, dicho. tome ramosa; caulibus pluribus in orbem dispositis, procumbentibus v. adscendentibus, palmaribus ac sesquipedalibus, tenuius crassiusve filiformilus, albidis, demum cum reliquis partibus plurimum purpurascentibus. Folia carnosula, semiuncialia et uncialia, 1-3 lineas lata, in petiolum 1-4 lineas longum, teretiusculum, filiformem rursus contracta, uninervia, subtus granulis linearibus, minutissimis, albidis, copiosissinis obsessa. Cymulae oppositifoliae 5_15florae, umbelliformes, ad cauliculorum et ramulorum nodos, internodiis $1 / 2 \ldots 2 \frac{1}{2}$ uncialibus discretos sessiles, $\mathbf{v}$. pedunculo 2_6 lineas longo suffultae. Pedicelli inaequales, plurimum 3_6 lineas longi, filiformes, laxiusculi. Calycis quinquepartiti laciniae late ovales, membranaceae, medio herbaceae, $1-1 / 2$ lineam longae, extus granulis adspersae, in fructu potissimum purpureae, demum expallescentes, stramineae. Stamina subhypogyna, calycem aequantia, filamentis e basi lata, plana, attenuatis, setaceis, antheris minutissimis, ovalibus, dilute flavis. Ovaria 5, uniovulata, discreta, stylis angulo interno infra apicem insertis, anthesi recurvis, in fructu persisten:ibus, uncinato-incurvis, filiformibus, facie interna longitudinaliter stigmatosis. Carpidia obovatolenticularia, calycem aequantia, membranacea, indehiscentia, papillosomuricata, ambitu crista purpurea, laciniata, mrjori lateralibasque duabus obsoletioribus, arcuatis, parallelis munita. Semina lenticularia, extremitate radiculari productiore, testa crustacea, nitida, atra. Embryo cyclicus, cotyledonibus incumbentibus. Albumen farinaceum. 
96. Finneam teleplaicines E.Meyer msc. L. suffruticosum, decumbens, squarroso-ramosum, glaberrimum; foliis subcoriaceis, aveniis, late ovalibus, ellipticis v. obovatis, obtusissimis, reflexomucronulatis, subsessilibus, alternis ; cymulis capituliformibus, terminalibus v. axillaribus, subsessilibus v. pedunculatis; calycis laciniis late ovalibus mucronulatis; staminibus 6 _ 7 basi fimbriato-barbatis; carpidiis reticulato-scrobiculatis, loculo spurio ad faciem comissuralem orbicularem aperto. Fen $\approx l$.

Limeum capense Ecklon et Zeyher Enum. pl. Afi. austr. n. 1830. (vix Thunb., quod dubium L. aphyllum Linnei.)

Patria. Cap. b. Spei. Hab. in rupibus ad ripas fuminis Gamka terrae mediae, regionem Koup dictam irrigantis (Drège! coll. pl. cap. sine no.); in locis caespitosis, alt. I-III., collium in Adon et ad Zwartkopsivier prov. Uitenhage, tum in Langekloof prov. George (Ecklon!).

Fruticulus, ut videtur, humifusus, squarrosus, ramis annotinis crassitie pennae corvinae, cinereis, digitalibus, plurimum tortis, florigeris simplicibus, filiformibus, virgatis, angulatis, digitalibus ac spithameis, confertius remotiusve foliosis, glaberrimis, pallide viridibus. Folia ab aliquibus lineis usque ad pollicem ab invicem dissita, firma, glaberrima, subtus nervo medio prominente percursa, supra sulco longitudinali exarata, $2 \frac{1}{2}-8$ lineas longa et $1 \frac{1}{2}-5$ lineas lata visa, in axillis par unicum foliorum minimorum patentissimorum frequentissime alentia. Florum cymulae capituli-v. glomeriformes, mole Pisi ad illam Juglandis, terminales $\mathbf{v}$. axillares, sessiles aut pedanculo rigido, aphyllo, ab aliquibus lineis ad semiunciam longo suffultae. Calycis laciniae medio herbaceae, dilutius profundiúsve virides, reliqua parte hyalino - memhranaceae, $1 \frac{1}{2}-2$ lineas longae, glabrae. Petala 5 , abortu subinde pauciora, calyce subbreviora, ovalia, obtusissima, integerrima, in ungniculum linearem lamina parum breviorem rursus angustata, nivea. Stamina 6_7, disco hypogyno inserta, petala aequantia, filamentis planis, e basi lata triangulari, margine crispato-ciliata subulatis. Styli duo minutissimi, clavato-filiformes. Carpidia 2 hemisphaerica, calycem subaequantia, reticulato-scrobiculata, profunde viridia, demum testacea, altero saepe hebetato, loculo spurio aperto.

9\%. Hinneam vigcostum Fenzl L. annuum, glanduloso-viscidum, diffuse-ramosum, humifusum; foliis petiolatis, suborbicularibus, late ovalibus ac obovatis, muticis; cymis glomeri-v. fasciculiformibus axillaribus ac terminalibus, sessilibus ac pedunculatis ; calycis laciniis suborbicularibus; petalis nullis; staminibus $6 \ldots 7$ basi imberbibus; carpidiorum reticulato - scrobiculatorum facie commissurali clausa, hemielliptica.

Syn. Gaudinia viscosa Gay in Bull. sc. nat. Vol, 18. (an. 1829.) p. 412.

\section{Patria: Senegambia (Sieber! Leprieur et Perrottet.)}

Herba valde ramosa, diffusa, pedalis visa, arena mobili adhaerente inquinata, tota pube glandulosa, viscida, minutissina dense velata, succulenta, laete virens. Cauliculi ac rami teretiusculi v. subangulati, crassitie fili emporctici mediocris. Folia $38^{\prime \prime \prime}$ longa, as: $2 \frac{1}{2}-4^{\prime \prime \prime}$ lata, basi 
in petiolum, lamina duplo triplove breviorem, filiformem rursus contracta, alterna et opposita, gemmulis axillaribus saepe facie subverticillata, carnosula, apice rotundata, mutica. Cymulae múutitiforae, globulosae, diametri 4-10 linearum, plurimum pedunculo 3-6 lineas longo suffultae, per ramos sparsae, oppositifoliae. Flores brevissime pedicellati. Calycis laciniae semimembranaceae, fere orbiculares, 1_z lineas longae. Carpidia reticulata, scrobiculata, versus margines incrassatos laevia.

OBSERVATIO : Species Limeo glomerato Eckl. et Zeyher ( $L$. Meyeri Fenzl in Annal. Wiener Mus. 1. 342.) proxima, foliis in petiolum haud attenuatis, latioribus, ac carpidiis hemiellipsoideis, nec hemisphaericis, reticulato-, nec echinulato-scrobicu* latis differt.

98. Anademia integrifolia Endl. in Annal. Wien. Mus. II. 209. A. foliis oblongis obtusis basi angustatis integris utrinque sericeis, spicis apicifloris.

In interioribus Novae-Hollandiae austro-occidentalis, ab oriente urbis New-York mense Decembri 1836 legit cl. Rö̈.

Ramuli compressiusculi, pilis sericeis vestiti. Folia alterna, pollicem dissita, oblonga v. obovato-oblonga, basi angustata, apice obtusa, bipollicaria, tres v. quatuor lineas lata, coriacea, margine integerrimo subincrassata, utrinque adpresse sericen-nitentia, obsolete trinervia. Spicae in apicibus ramulorum oppositifoliae, pedunculatae, duos v. tres pollices longae, densiflorae, ab apice ad basim florentes. Pedicelli tenuissimi, lineares, geminati, quantum e bracteae delapsae cicatrice judicare licet unibracteati. Perigonium glabrum, luteum, foliolis quatuor spathulatis, duas lineas longis, canaliculatis, apice concavis, laxiuscule patentibus. Antherae oblongae, foliolorum apicibus concavis immersae. Glandulae hypogynae omnino nullae. Ovarium pedicello vix dimidiam lineam longo, sub grossificatione paullulum elongato excentrice impositum, uniloculare. Ooula duo, an collateralia? quorum alterum sub grossificatione multo minus. Stylus filiformis, declinatus, ovario multo longior; stigna incrassatum, conicum, obtusiusculum.

99. Anademia filiformis Endl. l. c. A. foliis elongato-filiformibus obsolete canaliculatis subsericeis, apice callo obtuso deciduo, spicis basifloris.

In Nova-Hollandia austro-occidentali, inter Swan-River et King-Georges-Sound. (Rö̈.)

Rami pubescentes, subflexuosi. Folia alterna, filiformia, duos v. tres pollices longa, laxiuscula, sericeo-pubescentia, facie obsolete canaliculata, apice in callum brevem, acntum, deciduum desinentia, basi attenuata. Spicae in apicibus ramulorum laterales, pedunculatae, cylindricae, sesquipollicem v. duos pollices longae, densae, glabrae, a basi florentes. Pedicelli geminati, dimidia linea vix longiores. Bracteae delapsae. Flores nagnitudine, colore et structura is Anadeniae integrifoliae omnino similes Stigma conicum, apice subtruncatum. 
BRASSAIA Endl. Calyx tetraphyllus, foliolis imbricatis, aequalibus, deciduis. Corollae petala in calyptram subhemisphaericam, apice umbilicatam, longitudinaliter multistriatam, deciduam coalita. Stamina 13, disci cum ovarii basi connati margini inserta, uniseriala; filamenta libera, e basi latiore tereti-subulata, antherae introrsae, biloculares, lineares, dorso supra basim insertae, patentes, loculis longitudinaliter dehiscentibus. Ovarium turbinato-conicum, inferne disco staminifero adnato vestilum, superne longitudinaliter multicostatum, tredecimloculare, septis cuneatis, crassis. Ovula plurima, placentae centralis lobis lamelliformibus simplicibus, e loculorum angulo centrali exsertis affixa... Stigma sessile, radiatum, centro depressum, ambitu lobalum, lobis brevibus, cum ovarii costis continuis, primum conniventibus, demum erectis, apice truncato stigmatosis. Fructus . . - Arbor v. frutex Novae.Hollandiae tropicae; foliis in apice ramorum confertis, longe petiolatis, palmatim septem v. quatordecimfoliolatis, foliolis petioli apici dilatato complanato orbiculato radiatim insertis, petiolulatis, oblongis, subobtusis, basi subcordatis, remote subserratis, stipulis intrapetiolaribus integris, adnatis, e basi late ovata elongato-acuminatis, dense imbricatis, racemis terminalibus pluribus, pedicellis brevibus crassis, basi bracteae delapsae cicatrice notatis, apice glomerato dense plurifloris, per rhachim elongatam, simplicissimam, strictam . sparsis:

Genus insigne, Marcgraviae proximum', dicavi honoribus viri clarissimi Samuelis Brassai, apud Claudiopolitanos in Transylvania Botanicam profitentis, qui solus, popularibus suis tritam a Linneanis viam adusque terentibus, rei herbariae methodum naturalem libris praeclaris, hungarico sermone conscriptis, adserere conatur.

\section{4 -106.}

100. Brassaia àctinophylla Endlicher Iconograph. $t$.

\section{t. 334 _336.) \\ Crescit in Nova-Hollandia tropica. (Ferd. Bauer Illustr.}

Folia in apicibus ramulorum alterna, conferta, longe petiolata, palmatim septem ad qualuordecimfoliolata. Stipulae intrapetiolares duos ad quatuor pollices longae, e basi lata semipollicari longe acuminatae, inferne facie concavac, dorsó subconvexae, dense imbricatae, apice subpatentes. Petioli semiteretes, sex-octopollicares, inferne incrassati, stipularum dorso paullo supra basim inserti, patentes v. foliorum pondere subdeflexi. Foliola 7-14, petiolorum apici complanato orbiculato, subdilatato petiolulis sesquipollicaribus radiatim inserta, oblonga, apice subobtusa, basi obsolete emarginata, sex v. octo polliceslonga, tres pollices lata, penninervia, remote et obsolete serrulata, coriacea, glaberrima, nitida. Kacemi terminales terni, basi vaginis stipularibus margine incisis muniti, simplices, stricti, digiti indicis circiter crassitie, quadri-sexpedales. Flores Pisi minoris mole, in apice pedicellorum simplicissimorum, pollicem longitudine aequantium, per rhachim conferte sparsorum capitato glomerati. Pedicelli hasi bracteae latae, semicircularis cicatrice notati. Calyx tetraphyllus, foliolis ovatis, acutiusculis, imbricatis, mature delabentibus. Corolla calyptrac- 
formis decidua, subcoriacea, subhemisphaerica, centro umbilicatim impressa, juxta longitudinem striis tredecim e petalorum suturis exarata. Discus urceolaris carnosulus, ovario usque admediam longitudinem alnatas, limbo nonnihil incrassato et truncatostaminifer. Stamina tredecim (constanter?) discilimbo intus inserta, in alabastro erecta, corollae suturis opposita, ejusdem longitudine, modico deinde incremento eandem dejicientia. Filamenta e basi latiore subulata, teretiuscula, erecta, demum subpatentia. Antherae oblongo-lineares, crassiusculae, dorso paullo infra medium insertae, primum erectae, demum fere horizontaliter patentes. Loculi oppositi, paralleli, contigui, intus juxta totam longitudinem dehiscentes. Ovarium subturbinato - conicum, basi cum disco staminifero connata teres, apice libero longitudinaliter tredecimcostatum, costis cum cicatricibus e staminum insertione impressis alternantibus, in lobulos breves, primum circa verticem depressum conniventes, demum erectos, apice truncato subdilatato stigmatosos, continuatis, intus tredecimloculare, septis crassis, cuneatis, loculis angastissimis. Ovula in placentis lamelliformibus, e loculorum angulo centrali exsertis, fere ad parietem pertingentibus, medio crassioribus, sectione horizontali longe ellipticis, utrinque plurime, minutissima. 


\section{INDEX DECADIS I-X.}

Anadenia filiformis Endl. n. 99. integrifolia Endl, n. 98.

Ancistrostigma cypseleoides Fenzl. n. 94 .

Anthocercis anisantha $E n d l . n .80$.

Anthotroche pannosa Endl.n.9.

Anticharis arabica Endl. n. 28 .

Aphanopetalum resinosum Endl. n. 42 .

Billardiera grandiflora Putt. $n$. 53. latifolia Putt. n. 52 .

Boronia ternatá Endl. n. 7.

Bossiaea nummularia Endl. n. 27. _ ovalifolia Endl. n. 26.

Brassaia actinophylla Endl. $n, 100$.

Cesatia ornata Endl. n. 71.

Chamaelaucium virgatum Endl. $n .84$.

Coclostylis loganioides Torr. et $A$. Gr. n. 41 .

Conomitra linearis Fenzl. n. 74.

Conospermum Hügelii $R, B r, n .66$. - - - laniflorum Endl.n.67.

- _ - Stoechadis Endl.n.68.

Conostylis candicans Endi. n. 23, juncea Endl. n. 22,

Corethrostylis bracteata Endl. $n .1$. Corrigiola telephivides Pöpp. n. 93.

Crossopteryx Kotschyana $F$ enzl. $n .51$.

Crumenaria erecta Reiss. $n$. 35 .

Cryptandra pomaderroides Reiss, n.36. Cycnogeton Hügelii Endl. $n$. 89.

Dasymalla axillaris Endl. n. 12. terminalis Endl. n. 13.

Daviesia alternifolia Endl. n. 54.

- - macrophylla Endl. n, 18,

- - oppositifolia Endl. n. 56.

- ternata Endl. n.55.

Didymanthus Roëi Endl. n. 10.

Dillwynia adenophora Endl. n. 30.

- - elegans Endl. n. 15.

- - filifolia Endl. n. 16.

$-$
Diplochonium sesuvioides Fenzl. n.65.

Dodonaea humilis Endl. n. 33 .

Eremophila víscida Endl. $n .59$.

Friedrichsthalia physaloides Fenzl. n. 61 .

Fockea capensis Endl, $n, 21$.

Gaudinia viscosa Gay. n. 97.

Gieseckia Miltus Fenzl. n. 95.

Glischrocaryon Roëi Endl. n. 88.

Glossodia Brunonis Endl. n. 20.

Grevillea Thelemanniana Hüg. $n .8$.

Halgania anagalloides Endl. n. 62.

- - integerrima Endl. n. 63.

- Lavandulacea Endl. $n .64$.

Jacksonia floribunda Endl. $n .87$.

Irlbachia Bonplandiana Fenzl.n. 14.

Kochia salsoloides Fenzl. n. 85 .

Kotschya africana Endl. n. 6.

Leeuwenhoeckia tiliacea E. Mey. n. 45.

Leucopogon corifolius Endl. n. 19.

Limeum telephioides E. Mey. n. 96. viscosim Fenzl. n. 97.

Mallophora corymbosa Endl. $n$. 73. globiflora Endl. n. 72.

Manglesia tridentifera Endl. $n, 31$. vestita Endl. n. 32.

Marianthus erubescens Putt. $n$. 69. floribundus Putt. $n .70$.

Miltus africana Lour. n. 95.

Monocosmia corrigioloides Fenzl.n.93.

Oncosporum bicolor Putt. n. 11.

Oxylobium spectabile Endl. n. 2.

Petalopogon cuspidatus heiss. n. 92.

Pholidia resinosa Endl. n. 58 .

Phylica cuspidata Eckl, et Zeyh.n.92.

Pileanthus peduncularis Endl, n. 83. Pittosporum densiflorum Putt. $n$. 50 .

- - Hügelianun Putt.n. 49.

Plinthus cryptocarpus Fenzl. n. 60.

Ploesslea floribunda Endl. $n .47$.

Polyzone purpurea Endl. n. 18.

Pomaderris hirta Reiss. $n .37$. 
Pultenaea rosmarinifolia Endl. n. 5. Thomasia macrocarpa Hüg. n. 40. Russeggera collina Endl. $n$. $46 . \quad$ Thryptomene australis Endl. $n$. 82. Schwabea modesta Endl. n. 91. Semonvillea fenestrata Fenzl. n.48. Silene thysanodes Fenzl. $n .39$. Soulangia;oppositifolia Reiss. n. 19. Spadostyles Benthamii Endl. n. 4 .

- _ concolor Endl. n. 24.

- - Hügelii Endl. n. 3. - - ramulosa Endl. n. 25.

Stenochilus racemosus Endl. $n .57$. Talinum monandrum R. et S. $n$. 93 . Thelymitra flexuosa Enill. n. 29.

Tribonanthes australis Endl. n. 34

Ungnadia speciosa Endl, n. 86.

Verticordia chrysantha Endl. $n$. 78. - - compta Endl. n. 75.

- - grandiflora Endl. n. 79.

- - picta Endl.n.77.

— _ Roëi Endl. n. 76.

Xeropetalum minus Endl. n. 4'. multiflorum Endl. n. 43. tiliaceum Endl. n. 45. Zizyphus Gardneri Reiss. n. 3s. 



